\title{
$N$-homogeneous superalgebras
}

\author{
Phùng Hô Hai*, Benoît Kriegk, and Martin Lorenz**
}

\begin{abstract}
We develop the theory of $N$-homogeneous algebras in a super-setting, with particular emphasis on the Koszul property. To any Hecke operator $\mathcal{R}$ on a vector superspace, we associate certain superalgebras $S_{\mathcal{R}, N}$ and $\Lambda_{\mathcal{R}, N}$ generalizing the ordinary symmetric and Grassmann algebra, respectively. We prove that these algebras are $N$-Koszul. For the special case where $\mathcal{R}$ is the ordinary supersymmetry, we derive an $N$-generalized super-version of MacMahon's classical "master theorem".
\end{abstract}

Mathematics Subject Classification (2000). 16S37, 05A19.

Keywords. Superalgebra, generalized Koszul algebra, $N$-homogeneous algebra, Hecke algebra, MacMahon's master theorem, binomial identity, Berezinian.

\section{Contents}

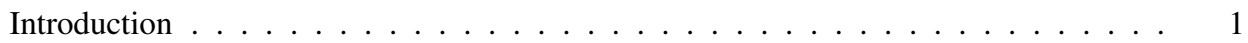

1 Review of linear superalgebra . . . . . . . . . . . . . . . . . . . . . . . . . . .

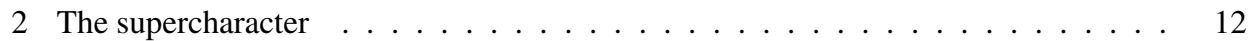

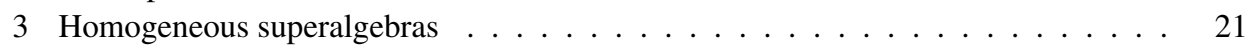

$4 N$-Koszul superalgebras . . . . . . . . . . . . . . . . . . . . . . . . . . . . . . . .

5 Koszul duality and master theorem . . . . . . . . . . . . . . . . . 41

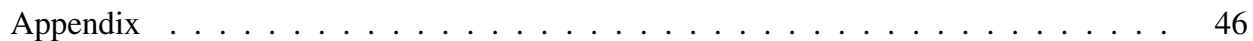

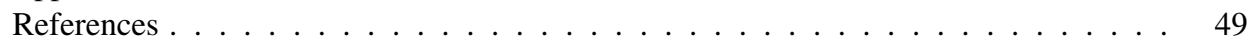

\section{Introduction}

1. The theory of $N$-homogeneous algebras owes its existence primarily to the concerns of noncommutative geometry. In fact, as has been expounded by Manin in his landmark publications [36], [37], quadratic algebras (the case $N=2$ ) provide a

\footnotetext{
${ }^{*}$ PHH is supported by the DFG through a Heisenberg-Fellowship.

** ML's research is supported in part by NSA Grants H98230-05-1-0025 and H98230-07-1-0008 and by Leverhulme Research Interchange Grant F/00158/X.
} 
convenient framework for the investigation of quantum group actions on noncommutative spaces. Moreover, certain Artin-Schelter regular algebras [1], natural noncommutative analogs of ordinary polynomial algebras, can be presented as associative algebras defined by cubic relations $(N=3)$. The latter algebras, as well as many of the quadratic algebras studied by Manin, enjoy the additional "Koszul property" which will be of central importance in the present article; it will be reviewed in detail in 6 below.

Motivated by these examples and others, Berger [5] initiated the systematic investigation of $N$-homogeneous algebras for all $N \geq 2$, introducing in particular a natural extension of the notion of Koszul algebra from the familiar quadratic setting to general $N$-homogeneous algebras. Article [5] gives examples of $N$-Koszul algebras for all $N \geq 2$; these are the so-called $N$-symmetric algebras, the special case $N=2$ being the ordinary symmetric (polynomial) algebra. Following the general outline of Manin's lecture notes [37] on the case of quadratic algebras, Berger, Dubois-Violette and Wambst developed the categorical aspects of $N$-homogeneous algebras in [7].

2. Current interest in $N$-homogeneous algebras is fueled in part by the fact that they do occur naturally in mathematical physics and in combinatorics. Indeed, Connes and Dubois-Violette [10], [11] introduced a class of 3-homogeneous algebras, called Yang-Mills algebras, which are in fact 3-Koszul. There are two versions of YangMills algebras: in the language of linear superalgebra, the first kind has even (parity $\overline{0})$ algebra generators while the second kind is generated by odd (parity $\overline{1}$ ) elements.

Combinatorics enters the picture via MacMahon's celebrated "master theorem" [35], specifically the recent quantum generalization of the master theorem due to Garoufalidis, Lê and Zeilberger [20]. As has been pointed out by two of the present authors in [28], the yoga of (quadratic) Koszul algebras leads to a rather effortless and conceptual proof of the quantum master theorem based on the fact that a certain quadratic algebra, known as quantum affine space, is Koszul. Further quantum generalizations and super-versions of the master theorem have been obtained by several authors using a variety of approaches; see Foata and Han [17], [18], [19], Konvalinka and Pak [33], Etingof and Pak [16].

3. From an algebraic point of view, MacMahon's master theorem (MT) in its various incarnations finds its most natural explanation by the phenomenon of "Koszul duality". Indeed, all versions of MT can be expressed in the form that, for some algebra $\mathcal{B}$, an equation $\Sigma_{1} \cdot \Sigma_{2}=1$ holds for suitable power series $\Sigma_{1}, \Sigma_{2} \in \mathcal{B} \llbracket t \rrbracket$. Here is a brief outline how one can arrive at such an equation starting with a given $N$ Koszul algebra $\mathcal{A}$. Associated with $\mathcal{A}$, there is a graded complex, $\mathrm{K}(\mathcal{A})$, which is exact in positive degrees, and a certain endomorphism bialgebra, end $\mathcal{A}$, which coacts on all components of $\mathrm{K}(\mathcal{A})$. These components therefore define elements of the representation ring $R_{\text {end }} \mathcal{A}$ of end $\mathcal{A}$, and exactness of $\mathrm{K}(\mathcal{A})$ in positive degrees yields 
an equation in the power series ring $R_{\text {end }} \mathcal{A} \llbracket t \rrbracket$. Due to the specific form of $\mathrm{K}(\mathcal{A})$, which is constructed from $\mathcal{A}$ together with its so-called dual algebra $\mathcal{A}^{!}$, the equation in question does indeed state that $\rho_{1} \cdot \rho_{2}=1$ holds for suitable $\rho_{1}, \rho_{2} \in R_{\text {end }} \mathcal{A} \llbracket t \rrbracket$. The last step in deriving a MT for $\mathcal{A}$ consists in using (super-)characters to transport

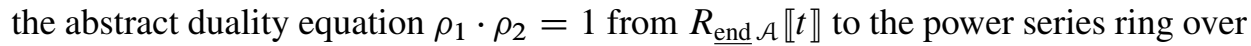
the algebra end $\mathcal{A}$, where it takes a more explicit and useable form. Here then is the flow chart of our approach:

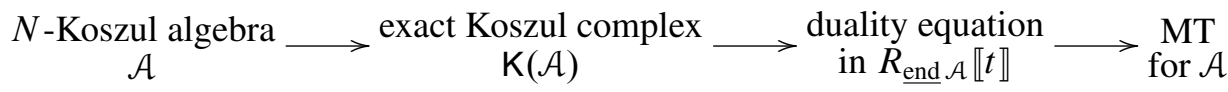

The actual labor involved in this process consists in the explicit evaluation of (super-)characters at the last arrow above. This step is often facilitated by specializing the bialgebra end $\mathcal{A}$, which is highly noncommutative, to a more familiar algebra $\mathcal{B}$ via a homomorphism end $\mathcal{A} \rightarrow \mathcal{B}$. For example:

- MacMahon's original MT [35] follows in the manner described above by starting with $\mathcal{A}=\mathcal{O}\left(\mathbb{k}^{d}\right)=\mathbb{k}\left[x_{1}, \ldots, x_{d}\right]$, the ordinary polynomial algebra or "affine space", and restricting the resulting MT over end $\mathcal{O}\left(\mathbb{k}^{d}\right)$ to the coordinate ring of $d \times d$-matrices, $\mathcal{O}\left(\operatorname{Mat}_{d}(\mathbb{k})\right)=\mathbb{k}\left[x_{j}^{i} \mid 1 \leq \overline{i, j} \leq d\right]$.

- As was explained in [28], taking "quantum affine space" $\mathcal{O}_{\boldsymbol{q}}\left(\mathbb{k}^{d}\right)$ as the point of departure one arrives at the quantum MT of Garoufalidis, Lê and Zeilberger [20] (and Konvalinka and Pak [33] in the multi-parameter case). The endomorphism bialgebra of $\mathcal{O}_{\boldsymbol{q}}\left(\mathbb{k}^{d}\right)$ is exactly the algebra of right-quantum matrices as defined in [20].

- Berger's $N$-symmetric algebra [5] leads to the $N$-generalization of the MT proved by Etingof and Pak [16] using the above approach, again after restricting to $\mathcal{O}\left(\mathrm{Mat}_{d}(\mathbb{k})\right)$.

4. The present article aims to set forth an extension of the existing theory of $N$ homogeneous algebras to the category Vect $_{\mathbb{k}}^{s}$ of vector superspaces over some base field $k$. While this does not give rise to principal obstacles given that [37] and [7] are at hand as guiding references, the setting of superalgebra requires careful consideration of the order of terms and the so-called "rule of signs" will be ubiquitous in our formulæ. In view of the potential interdisciplinary interest of this material, we have opted to keep our presentation reasonably self-contained and complete.

Therefore, in Sections 1 and 2, we deploy the requisite background material from superalgebra in some detail before turning to $N$-homogeneous superalgebras in Section 3. The latter section, while following the general outline of [37] and [7] rather closely, also offers explicit discussions of a number of important examples. We interpolate the pure even and pure odd Yang-Mills algebras defined by Connes and Dubois-Violette [10], [11] by a family of superalgebras $\operatorname{y\mathcal {M}}^{p \mid q}$ and give a unified 
treatment of these algebras. (It turns out, however, that the mixed algebras $\operatorname{y\mathcal {M}}^{p \mid q}$, with $p$ and $q$ both nonzero, are less well-behaved than the pure cases.) Moreover, we discuss a superized version of the $N$-symmetric algebras of Berger [5]. Finally, in Example 3.4, we introduce new $N$-homogeneous super-versions of the symmetric algebra and the Grassmann algebra of a vector superspace $V$; these are associated with any Hecke operator $\mathcal{R}: V^{\otimes 2} \rightarrow V^{\otimes 2}$ and will be denoted by $\mathrm{S}_{\mathcal{R}, N}$ and $\Lambda_{\mathcal{R}, N}$, respectively.

Sections 4 and 5 contain our main results: Theorem 4.5 shows that the superalgebras $\mathrm{S}_{\mathcal{R}, N}$ and $\Lambda_{\mathcal{R}, N}$ are in fact $N$-Koszul, and Theorem 5.4 is superized version of the aforementioned $N$-generalized MT of Etingof and Pak [16], Theorem 2. The special case $N=2$ of Theorem 5.4 is a superization of the original master theorem of MacMahon [35]. The present article was motivated in part by a comment in Konvalinka and Pak [33], 13.4, asking for a "real" super-analog of the classical MT.

5. A considerable amount of research has been done by mathematical physicists on various quantum matrix identities. Some of these investigations have been carried out in a super-setting; see, e.g., Gurevich, Pyatov and Saponov [23], [24] and the references therein. However, the techniques employed in these articles appear to be quite different from ours.

After submitting this article, we also learned of recent work of Konvalinka [31], [32] which not only concerns MacMahon's MT but also other matrix identities such as the determinantal identity of Sylvester. These identities are proved in [31], [32] by combinatorial means in various noncommutative settings including the right-quantum matrix algebra end $\mathcal{O}_{q}\left(\mathbb{k}^{d}\right)$.

6. We conclude this Introduction by reviewing the precise definitions of $N$-homogeneous and $N$-Koszul algebras. Our basic reference is Berger [5]; see also [2], [7], [21].

Let $\mathcal{A}$ be a connected $\mathbb{Z}_{\geq 0}$-graded algebra over a field $\mathbb{k}$; so $\mathcal{A}=\bigoplus_{n \geq 0} \mathcal{A}_{n}$ for k-subspaces $\mathcal{A}_{n}$ with $\mathcal{A}_{0}=\mathbb{k}$ and $\mathcal{A}_{n} \mathcal{A}_{m} \subseteq \mathcal{A}_{n+m}$. Choose a minimal generating set for the algebra $\mathcal{A}$ consisting of homogeneous elements of positive degree; this amounts to choosing a graded basis for a graded subspace $V \subseteq \mathcal{A}_{+}=\bigoplus_{n>0} \mathcal{A}_{n}$ such that $\mathcal{A}_{+}=\mathcal{A}_{+}^{2} \oplus V$. The grading of $V$ imparts a grading to the tensor algebra $\mathrm{T}(V)$ of the space $V$, and we have a graded presentation

$$
\mathrm{T}(V) / I \stackrel{\sim}{\longrightarrow} \mathcal{A}
$$

for some graded ideal $I$ of $\mathrm{T}(V)$, the ideal of relations of $\mathcal{A}$.

Recall that a graded vector space $M=\bigoplus_{n \in \mathbb{Z}} M_{n}$ is said to live in degrees $\geq n_{0}$ if $M_{n}=0$ for all $n<n_{0}$. Note that the relation ideal $I$ lives in degrees $\geq 2$, because $\mathrm{T}(V)_{0} \oplus \mathrm{T}(V)_{1} \subseteq \mathbb{k} \oplus V$ and $\mathbb{k} \oplus V$ injects into $\mathcal{A}$. Fix an integer $N \geq 2$ and define 
the jump function

$$
v_{N}(i)= \begin{cases}\frac{i}{2} N & \text { if } i \text { is even } \\ \frac{i-1}{2} N+1 & \text { if } i \text { is odd. }\end{cases}
$$

The following proposition is identical with [8], Proposition 2.1, except for the fact that we do not a priori assume $\mathcal{A}$ to be generated in degree 1 . A proof is given in the Appendix.

Proposition. The ideal I of relations of $\mathcal{A}$ lives in degrees $\geq N$ if and only if $\operatorname{Tor}_{i}^{\mathcal{A}}(\mathbb{k}, \mathbb{k})$ lives in degrees $\geq v_{N}(i)$ for all $i \geq 0$.

Following Berger [5], the graded algebra $\mathcal{A}$ is said to be $N$-Koszul if $\operatorname{Tor}_{i}^{\mathcal{A}}(\mathbb{k}, \mathbb{k})$ is concentrated in degree $v_{N}(i)$ for all $i \geq 0$. This implies that the space of algebra generators $V$ is concentrated in degree $v_{N}(1)=1$; so the algebra $\mathcal{A}$ is 1-generated. Moreover, choosing a minimal set of homogeneous ideal generators for the relation ideal $I$ amounts to choosing a graded basis for a graded subspace $R \subseteq I$ such that

$$
I=R \oplus(V \otimes I+I \otimes V) .
$$

Then $\operatorname{Tor}_{2}^{\mathcal{A}}(\mathbb{k}, \mathbb{k}) \cong R$ and so $R$ must be concentrated in degree $v_{N}(2)=N$ when $\mathcal{A}$ is $N$-Koszul. To summarize, all $N$-Koszul algebras are necessarily 1 -generated and they have defining relations in degree $N$; so there is a graded isomorphism

$$
\mathcal{A} \cong \mathrm{T}(V) /(R) \quad \text { with } R \subseteq V^{\otimes N} .
$$

Such algebras are called $N$-homogeneous.

We remark that Green et al. [21] have studied $N$-Koszul algebras in the more general context where the grading $\mathcal{A}=\bigoplus_{n \geq 0} \mathcal{A}_{n}$ is not necessarily connected $\left(\mathcal{A}_{0}=\mathbb{k}\right)$. In [21], Theorem 4.1, it is shown that an $N$-homogeneous algebra $\mathcal{A}$ with $\mathcal{A}_{0}$ split semisimple over $\mathbb{k}$ is $N$-Koszul if and only if the Yoneda Ext-algebra $E(\mathcal{A})=\bigoplus_{n \geq 0} \operatorname{Ext}_{\mathcal{A}}^{n}\left(\mathcal{A}_{0}, \mathcal{A}_{0}\right)$ is generated in degrees $\leq 2$.

Any $N$-homogeneous algebra $\mathcal{A}$ whose generating space $V$ carries a $\mathbb{Z}_{2}$-grading and whose defining relations $R$ are $\mathbb{Z}_{2}$-graded is naturally a $\mathbb{k}$-superalgebra, that is, $\mathcal{A}$ has a $\mathbb{Z}_{2}$-grading ("parity") besides the basic $\mathbb{Z}_{\geq 0}$-grading ("degree"). As will be reviewed below, this extra structure provides us with additional functions on Grothendieck rings, namely superdimension and supercharacters, which lead to natural formulations of the MT in a superized context. Note, however, that the defining property of $N$-Koszul algebras makes no reference to the $\mathbb{Z}_{2}$-grading of $\mathcal{A}$. Thus, an $N$-homogeneous superalgebra is Koszul precisely if it is Koszul as an ordinary $N$-homogeneous algebra (forgetting the $\mathbb{Z}_{2}$-grading).

7. Throughout $\mathbb{k}$ is a commutative field and $\otimes$ stands for $\otimes_{\mathbb{k}}$. Scalar multiplication in $\mathbb{k}$-vector spaces will often, but not always, be written on the right while linear maps will act from the left. We tacitly assume throughout that char $k \neq 2$; further restrictions on the characteristic of $\mathbb{k}$ will be stated when required. 


\section{Review of linear superalgebra}

1.1. Vector superspaces. A vector superspace over $\mathbb{k}$ is a $\mathbb{k}$-vector space $V$ equipped with a grading by the group $\mathbb{Z}_{2}=\mathbb{Z} / 2 \mathbb{Z}=\{\overline{0}, \overline{1}\}$. Thus, we have a decomposition $V=V_{\overline{0}} \oplus V_{\overline{1}}$ with $\mathbb{k}$-subspaces $V_{\overline{0}}$ and $V_{\overline{1}}$ whose elements are called even and $o d d$, respectively. In general, the $\mathbb{Z}_{2}$-degree of a homogeneous element $a \in V$ is also called its parity; it will be denoted by $\hat{a} \in \mathbb{Z}_{2}$. Vector superspaces over $\mathbb{k}$ form a category Vect $t_{\mathbb{k}}^{s}$ whose morphisms are given by the linear maps preserving the $\mathbb{Z}_{2}$-grading; such maps are also called even linear maps.

The dimension of an object $V$ of $\operatorname{Vect}_{\mathbb{k}}^{S}$ is the usual $\mathbb{k}$-linear dimension. We shall use the notation

$$
d=\operatorname{dim}_{\mathbb{k}} V, p=\operatorname{dim}_{\mathbb{k}} V_{\overline{0}} \quad \text { and } \quad q=\operatorname{dim}_{\mathbb{k}} V_{\overline{1}} .
$$

So $d=p+q$. The superdimension of a vector superspace $V$ with $d<\infty$ is defined by

$$
\operatorname{sdim} V=p-q \in \mathbb{Z} .
$$

When working with a fixed basis $\left\{x_{i}\right\}$ of a given $V$ in $\operatorname{Vect}_{\mathbb{k}}^{s}$ we shall assume that each $x_{i}$ is homogeneous; the parity of $x_{i}$ will be denoted by $\hat{i}$. The basis $x_{1}, x_{2}, \ldots$ is called standard if $\hat{i}=\overline{0}(i \leq p)$ and $\hat{i}=\overline{1}(i>p)$.

1.2. Tensors. The tensor product $U \otimes V$ of vector superspaces $U$ and $V$ in $\operatorname{Vect}_{\mathbb{k}}^{S}$ is the usual tensor product over $\mathbb{k}$ of the underlying vector spaces equipped with the natural $\mathbb{Z}_{2}$-grading: if $a, b$ are homogeneous elements then the parity of $a \otimes b$ is $\hat{a}+\hat{b} \in \mathbb{Z}_{2}$. Instead of the usual symmetry isomorphism $U \otimes V \stackrel{\sim}{\longrightarrow} V \otimes U$ for interchanging terms in a tensor product we shall use the rule of signs, that is, the following functorial supersymmetry isomorphism in $\operatorname{Vect}_{\mathbb{k}}^{s}$ :

$$
c_{U, V}: U \otimes V \stackrel{\sim}{\longrightarrow} V \otimes U, \quad u \otimes v \mapsto(-1)^{\hat{\imath} \hat{v}} v \otimes u
$$

for $u, v$ homogeneous. (All formulas stated for homogeneous elements only are to be extended to arbitrary elements by linearity.) The supersymmetry isomorphisms $c_{U, V}$ satisfy $c_{V, U} \circ c_{U, V}=\operatorname{Id}_{U \otimes V}$, and they are compatible with the usual associativity isomorphims $a_{U, V, W}:(U \otimes V) \otimes W \cong U \otimes(V \otimes W)$ in $\operatorname{Vect}_{\mathbb{k}}^{S}$, that is, they satisfy the "Hexagon Axiom"; see [29], Def. XIII.1.1. Therefore, Vects is a symmetric tensor category; the unit object is the field $k$, with parity $\overline{0}$. See [29], Chap. XIII, or [12] for background on tensor categories.

1.3. Homomorphisms. The space $\operatorname{Hom}_{\mathbb{k}}(V, U)$ of all $\mathbb{k}$-linear maps between vector superspaces $V$ and $U$ is again an object of $\operatorname{Vect}_{\mathbb{k}}^{S}$, with grading

$$
\operatorname{Hom}_{\mathbb{k}}(V, U)_{\overline{0}}=\operatorname{Hom}_{\mathbb{k}}\left(V_{\overline{0}}, U_{\overline{0}}\right) \oplus \operatorname{Hom}_{\mathbb{k}}\left(V_{\overline{1}}, U_{\overline{1}}\right)
$$


and

$$
\operatorname{Hom}_{\mathbb{k}}(V, U)_{\overline{1}}=\operatorname{Hom}_{\mathbb{k}}\left(V_{\overline{0}}, U_{\overline{1}}\right) \oplus \operatorname{Hom}_{\mathbb{k}}\left(V_{\overline{1}}, U_{\overline{0}}\right)
$$

so

$$
\operatorname{Hom}_{\mathbb{k}}(V, U)_{\overline{0}}=\operatorname{Hom}_{V_{\text {ect }}^{s}}(V, U) .
$$

In particular, the linear dual $V^{*}=\operatorname{Hom}_{\mathbb{k}}(V, \mathbb{k})$ belongs to $\operatorname{Vect}_{\mathbb{k}}^{S}$. Given homogeneous bases $\left\{x_{j}\right\}$ of $V$ and $\left\{y_{i}\right\}$ of $U$ we can describe any $f \in \operatorname{Hom}_{\mathbb{k}}(V, U)$ by its matrix $F=\left(F_{j}^{i}\right)$ :

$$
f\left(x_{j}\right)=\sum_{i} y_{i} F_{j}^{i} .
$$

If $f$ is an even map then $F_{j}^{i}=0$ unless $\hat{i}+\hat{j}=\overline{0}$.

For finite-dimensional vector superspaces, we have the following functorial isomorphisms in Vect $_{\mathbb{k}}^{S}$ (see, e.g., [43], I.8):

$$
U \otimes V^{*} \cong \operatorname{Hom}_{\mathbb{k}}(V, U)
$$

via $(u \otimes f)(v)=u\langle f, v\rangle$, and

$$
V_{1}^{*} \otimes \cdots \otimes V_{m}^{*} \cong\left(V_{m} \otimes \cdots \otimes V_{1}\right)^{*}
$$

via $\left\langle f_{1} \otimes \cdots \otimes f_{m}, v_{m} \otimes \cdots \otimes v_{1}\right\rangle=\prod_{i}\left\langle f_{i}, v_{i}\right\rangle$. Here, we use the notation $\langle f, v\rangle=$ $f(v)$ for the evaluation pairing

$$
\mathrm{ev}_{V}=\langle\cdot, \cdot\rangle: V^{*} \otimes V \rightarrow \mathbb{k}
$$

in $\operatorname{Vect}_{\mathbb{k}}^{S}$. Similarly, we have a pairing

$$
V \otimes V^{*} \stackrel{c_{V, V^{*}}}{\longrightarrow} V^{*} \otimes V \stackrel{\mathrm{ev}_{V}}{\longrightarrow} \mathbb{k}
$$

which yields an isomorphism

$$
V \stackrel{\sim}{\longrightarrow} V^{* *}
$$

in $\operatorname{Vect}_{\mathbb{k}}^{S}$.

The isomorphism (1.3) (which is valid as long as one of $U$ or $V$ is finite-dimensional) has the following explicit description. Fix homogeneous bases $\left\{x_{j}\right\}$ of $V$ and $\left\{y_{i}\right\}$ of $U$ and let $F=\left(F_{j}^{i}\right)$ be the matrix of a given $f \in \operatorname{Hom}_{\mathbb{k}}(V, U)$ with respect to these bases, as in (1.2). Let $\left\{x^{j}\right\}$ be the dual basis of $V^{*}$, defined by $\left\langle x^{j}, x_{\ell}\right\rangle=\delta_{\ell}^{j}$ (Kronecker delta). Then the image of $f$ in $U \otimes V^{*}$ is given by $\sum_{i, j} y_{i} \otimes x^{j} F_{j}^{i}$. Note also that $x_{i}$ and $x^{i}$ have the same parity.

Finally, if $U, V$ and $W$ are vector superspaces, with $U$ finite-dimensional, then the isomorphism Id $\otimes c_{W, U^{*}}: V \otimes W \otimes U^{*} \stackrel{\sim}{\longrightarrow} V \otimes U^{*} \otimes W$ together with (1.3) yields an isomorphism

$$
\operatorname{Hom}_{\mathbb{k}}(U, V \otimes W) \stackrel{\sim}{\longrightarrow} \operatorname{Hom}_{\mathbb{k}}(U, V) \otimes W
$$


in $\operatorname{Vect}_{\mathbb{k}}^{S}$ which is explicitly given by $(f \otimes w)(u)=(-1)^{\hat{w} \hat{u}} f(u) \otimes w$. Similarly, for vector superspaces $U, U^{\prime}, V, V^{\prime}$ with $U, U^{\prime}$ finite-dimensional, there is an isomorphism

$$
\operatorname{Hom}_{\mathbb{k}}\left(U \otimes U^{\prime}, V \otimes V^{\prime}\right) \stackrel{\sim}{\longrightarrow} \operatorname{Hom}_{\mathbb{k}}(U, V) \otimes \operatorname{Hom}_{\mathbb{k}}\left(U^{\prime}, V^{\prime}\right)
$$

in $\operatorname{Vect}_{\mathbb{k}}^{S}$ given by $(f \otimes g)(u \otimes v)=(-1)^{\hat{g} \hat{u}} f(u) \otimes g(v)$.

1.4. Supertrace. Let $V$ be a finite-dimensional object of $\operatorname{Vect}_{\mathbb{k}}^{S}$. The supertrace is the map

$$
\text { str: } \operatorname{End}_{\mathbb{k}}(V) \underset{(1.3)}{\stackrel{\sim}{\longrightarrow}} V \otimes V^{*} \underset{(1.3)}{\longrightarrow} \mathbb{k}
$$

in $\operatorname{Vect}_{\mathbb{k}}^{s}$. In order to describe the supertrace in terms of matrices, fix a basis $\left\{x_{i}\right\}$ of $V$ consisting of homogeneous elements and let $F=\left(F_{j}^{i}\right)$ be the matrix of $f \in \operatorname{End}_{\mathbb{k}}(V)$ as in (1.2). Then

$$
\operatorname{str}(f)=\sum_{i}(-1)^{\hat{i}} F_{i}^{i}
$$

where $\hat{i}$ is the parity of $x_{i}$ (and of the dual basis vector $x^{i} \in V^{*}$ ) as in $\S 1.1$. Thus,

$$
\operatorname{str}\left(\operatorname{Id}_{V}\right)=\operatorname{sdim} V \cdot 1_{\mathbb{k}} .
$$

1.5. Action of the symmetric group. Given vector superspaces $V_{1}, \ldots, V_{n}$, we can consider the morphism

$$
c_{i}: V_{1} \otimes \cdots \otimes V_{i} \otimes V_{i+1} \otimes \cdots \otimes V_{n} \rightarrow V_{1} \otimes \cdots \otimes V_{i+1} \otimes V_{i} \otimes \cdots \otimes V_{n}
$$

in Vect $t_{\mathbb{k}}^{s}$ which interchanges the factors $V_{i}$ and $V_{i+1}$ via $c_{V_{i}}, V_{i+1}$ and is the identity on all other factors. More generally, for any $\sigma \in \mathfrak{S}_{n}$, the symmetric group consisting of all permutations of $\{1,2, \ldots, n\}$, one can define a morphism

$$
c_{\sigma}: V_{1} \otimes \cdots \otimes V_{n} \rightarrow V_{\sigma^{-1}(1)} \otimes \cdots \otimes V_{\sigma^{-1}(n)}
$$

in $\operatorname{Vect}_{\mathbb{k}}^{S}$ as follows. Recall that $\widetilde{S}_{n}$ is generated by the transpositions $\sigma_{1}, \ldots, \sigma_{n-1}$ where $\sigma_{i}$ interchanges $i$ and $i+1$ and leaves all other elements of $\{1,2, \ldots, n\}$ fixed. The minimal length of a product in the $\sigma_{i}$ 's which expresses a given element $\sigma \in \mathfrak{S}_{n}$ is called the length of $\sigma$ and denoted $\ell(\sigma)$; it is given by

$$
\ell(\sigma)=\# \operatorname{inv}(\sigma) \quad \text { with } \operatorname{inv}(\sigma)=\{(i, j) \mid i<j \text { but } \sigma(i)>\sigma(j)\} .
$$

Writing $\sigma \in \mathfrak{S}_{n}$ as a product of certain $\sigma_{i}$, the analogous product of the maps $c_{i}$ yields a morphism $c_{\sigma}$ as above. This morphism is independent of the way $\sigma$ is expressed in terms of the transpositions $\sigma_{i}$; see [43], I.4.13, or [29], Theorem XIII.1.3. If all $v_{i} \in V_{i}$ are homogeneous then

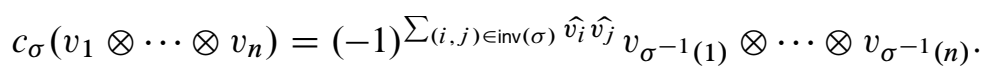


For example, if all $v_{i}$ are even then the \pm -sign on the right is + , and if all $v_{i}$ are odd then it is $\operatorname{sgn}(\sigma)$, the signature of $\sigma$.

Taking all $V_{i}=V$ we obtain a representation $c: \mathfrak{S}_{n} \rightarrow \operatorname{Aut}_{\mathrm{Vect}_{\mathrm{k}}^{s}}\left(V^{\otimes n}\right)$ where $V^{\otimes n}=V \otimes \cdots \otimes V$ ( $n$ factors). Letting $\mathbb{k}\left[\Im_{n}\right]$ denote the group algebra of the symmetric group, this extends uniquely to an algebra map

$$
c: \mathbb{k}\left[\widetilde{S}_{n}\right] \rightarrow \operatorname{End}_{\mathrm{Vect}_{\mathbb{k}}^{s}}\left(V^{\otimes n}\right)
$$

We will write $c_{a}:=c(a)$ for $a \in \mathbb{k}\left[\Im_{N}\right]$.

For the dual superspace $V^{*}$, in addition to the above representation $c: \mathbb{k}\left[\Im_{n}\right] \rightarrow$ $\operatorname{End}_{V_{\text {ect }}^{s}}\left(V^{* \otimes n}\right)$, we also have the contragredient representation

$$
c^{*}: \mathbb{k}\left[\widetilde{\Im}_{n}\right] \rightarrow \operatorname{End}_{\mathrm{Vect}_{\mathbb{k}}^{s}}\left(V^{* \otimes n}\right)
$$

for the pairing $\langle\cdot, \cdot\rangle: V^{* \otimes n} \otimes V^{\otimes n} \rightarrow \mathbb{k}$ in (1.4). Explicitly,

$$
\left\langle c_{a}^{*}(x), y\right\rangle=\left\langle x, c_{a^{*}}(y)\right\rangle
$$

for all $a \in \mathbb{k}\left[\Im_{n}\right], x \in V^{* \otimes n}$ and $y \in V^{\otimes n}$. Here, $\cdot^{*}: \mathbb{k}\left[\Im_{n}\right] \rightarrow \mathbb{k}\left[\Im_{n}\right]$ is the involution sending $\sigma \in \mathfrak{S}_{n}$ to $\sigma^{-1}$. These two representations are related by

$$
c_{a}^{*}=c_{\tau a \tau}
$$

where $\tau=(1, n)(2, n-1) \ldots \in \mathfrak{S}_{n}$ is the order reversal involution. One only needs to check (1.11) for the transpositions $a=\sigma_{i}$, which is straightforward.

1.6. Hecke algebras. We recall some standard facts concerning Hecke algebras; these are deformations of the group algebra $\mathbb{k}\left[\widetilde{\Im}_{n}\right]$ considered above. For details, see [13], [14].

Fix $0 \neq q \in \mathbb{k}$. The Hecke algebra $\mathscr{H}_{n, q}$ is generated as $\mathbb{k}$-algebra by elements $T_{1}, \ldots, T_{n-1}$ subject to the relations

$$
\begin{aligned}
& \left(T_{i}+1\right)\left(T_{i}-q\right)=0, \\
& T_{i} T_{i+1} T_{i}=T_{i+1} T_{i} T_{i+1}, \\
& T_{i} T_{j}=T_{j} T_{i} \quad \text { if }|i-j| \geq 2 .
\end{aligned}
$$

When $q=1$, one has an isomorphism $\mathscr{H}_{n, 1} \stackrel{\sim}{\longrightarrow}\left[\widetilde{S}_{n}\right], T_{i} \mapsto \sigma_{i}$, where $\sigma_{i}$ is the transposition $(i, i+1)$ as in $\S 1.5$. The algebra $\mathscr{H}_{n, q}$ has a $\mathbb{k}$-basis $\left\{T_{\sigma} \mid \sigma \in \mathfrak{S}_{n}\right\}$ so that

(i) $T_{\mathrm{Id}}=1$ and $T_{\sigma_{i}}=T_{i}$;

(ii) $T_{\sigma} T_{\sigma_{i}}= \begin{cases}T_{\sigma \sigma_{i}} & \text { if } \ell\left(\sigma \sigma_{i}\right)=\ell(\sigma)+1, \\ q T_{\sigma \sigma_{i}}+(q-1) T_{\sigma} & \text { otherwise. }\end{cases}$ 
By $k$-linear extension of the rule

$$
T_{\sigma}^{*}:=T_{\sigma^{-1}} \quad\left(\sigma \in \widetilde{S}_{n}\right)
$$

one obtains an involution ${ }^{*}: \mathscr{H}_{n, q} \rightarrow \mathscr{H}_{n, q}$. The elements $T_{i}^{\prime}:=-q T_{i}^{-1}=$ $q-1-T_{i}$ also satisfy relations (1.12). Therefore,

$$
\alpha\left(T_{i}\right):=-q T_{i}^{-1}
$$

defines an algebra automorphism $\alpha: \mathscr{H}_{n, q} \rightarrow \mathscr{H}_{n, q}$ of order 2 .

The Hecke algebra $\mathscr{H}_{n, q}$ is always a symmetric algebra, and $\mathscr{H}_{n, q}$ is a split semisimple $\mathbb{k}$-algebra iff the following condition is satisfied:

$$
[n]_{q} !:=\prod_{i=1}^{n}[i]_{q} \neq 0 \quad \text { where }[i]_{q}:=1+q+\cdots+q^{i-1} .
$$

More precisely, if (1.14) holds then

$$
\mathscr{H}_{n, q} \cong \bigoplus_{\lambda \vdash n} \operatorname{Mat}_{d_{\lambda} \times d_{\lambda}}(\mathbb{k})
$$

where $\lambda$ runs over all partitions of $n$ and $d_{\lambda}$ denotes the number of standard $\lambda$-tableaux. The only partitions $\lambda$ with $d_{\lambda}=1$ are $\lambda=(n)$ and $\lambda=\left(1^{n}\right)$. The central primitive idempotents of $\mathscr{H}_{n, q}$ for these partitions are given by

$$
X_{n}:=\frac{1}{[n]_{q} !} \sum_{\sigma \in \subseteq_{n}} T_{\sigma}
$$

and

$$
Y_{n}:=\frac{1}{[n]_{q^{-1}} !} \sum_{\sigma \in \mathbb{S}_{n}}(-q)^{-\ell(\sigma)} T_{\sigma} .
$$

These idempotents are usually called the q-symmetrizer and the q-antisymmetrizer, respectively. One has

$$
X_{n} T_{\sigma}=T_{\sigma} X_{n}=q^{\ell(\sigma)} X_{n} \quad \text { and } \quad Y_{n} T_{\sigma}=T_{\sigma} Y_{n}=(-1)^{\ell(\sigma)} Y_{n}
$$

for $\sigma \in \widetilde{S}_{n}$. Furthermore, $\alpha\left(X_{n}\right)=Y_{n}$.

For later use, we note the following well-known consequence of (1.18). If $M$ is any $\mathscr{H}_{n, q}$-module, with corresponding representation $\mu: \mathscr{H}_{n, q} \rightarrow \operatorname{End}_{\mathbb{k}}(M)$, then

$$
\operatorname{Im}\left(\mu\left(X_{n}\right)\right)=\bigcap_{i=1}^{n-1} \operatorname{Im}\left(\mu\left(T_{i}\right)+1\right) .
$$

Indeed, (1.18) implies that $X_{n}=[2]_{q}^{-1}\left(T_{i}+1\right) X_{n}$, which yields the inclusion $\subseteq$. On the other hand, any $m \in \bigcap_{i=1}^{n-1} \operatorname{Im}\left(\mu\left(T_{i}\right)+1\right)$ satisfies $\left(\mu\left(T_{i}\right)-q\right)(m)=0$ for all $i$, by (1.12). Therefore, $\mu\left(T_{\sigma}\right)(m)=q^{\ell(\sigma)} m$ holds for all $\sigma \in \widetilde{S}_{n}$, and hence $\mu\left(X_{n}\right)(m)=\frac{1}{[n]_{q} !} \sum_{\sigma \in \mathbb{S}_{n}} q^{\ell(\sigma)} m=m$. This proves $\supseteq$. 
1.7. Hecke operators. Again, let $0 \neq q \in \mathbb{k}$. A Hecke operator (associated to $q$ ) on a vector superspace $V$ is a morphism $\mathcal{R}: V^{\otimes 2} \rightarrow V^{\otimes 2}$ in Vect $t_{\mathbb{k}}^{S}$ satisfying the Hecke equation

$$
(\mathcal{R}+1)(\mathcal{R}-q)=0
$$

and the Yang-Baxter equation

$$
\mathcal{R}_{1} \mathcal{R}_{2} \mathcal{R}_{1}=\mathcal{R}_{2} \mathcal{R}_{1} \mathcal{R}_{2}
$$

where $\mathcal{R}_{1}:=\mathcal{R} \otimes \operatorname{Id}_{V}: V^{\otimes 3} \rightarrow V^{\otimes 3}$ and similarly $\mathcal{R}_{2}:=\operatorname{Id}_{V} \otimes \mathcal{R}$.

The Hecke equation implies that $\mathcal{R}$ is invertible. Moreover, if $\mathcal{R}$ is a Hecke operator associated to $q$ then so is $-q \mathcal{R}^{-1}$.

Defining $\rho\left(T_{i}\right):=\operatorname{Id}_{V}^{\otimes i-1} \otimes \mathcal{R} \otimes \operatorname{Id}_{V}^{\otimes n-i-1}$, one obtains a representation

$$
\rho=\rho_{n, \mathcal{R}}: \mathscr{H}_{n, q} \rightarrow \operatorname{End}_{\operatorname{Vect}_{\mathrm{k}}^{s}}\left(V^{\otimes n}\right) .
$$

The representations $\rho_{n, \mathcal{R}}$ and $\rho_{n,-q \mathcal{R}^{-1}}$ are related by $\rho_{n,-q \mathcal{R}^{-1}}=\rho_{n, \mathcal{R}} \circ \alpha$, where $\alpha$ is the automorphism of $\mathscr{H}_{n, q}$ defined in (1.13).

Example 1.1. The supersymmetry operator $c_{V, V}: V^{\otimes 2} \rightarrow V^{\otimes 2}$ in (1.1) is a Hecke operator associated to $q=1$, as is its negative, $-c_{V, V}$. The representation $\rho_{n, c_{V, V}}$ of $\mathscr{H}_{n, 1}=\mathbb{k}\left[\Theta_{n}\right]$ in (1.20) is identical with (1.10).

Example 1.2 (Superized Drinfel'd-Jimbo [38], [27]). Let $x_{1}, \ldots, x_{d}$ be a standard basis of $V$ as in $\S 1.1$. The super-analog $\mathcal{R}=\mathcal{R}^{D J}$ of the standard Drinfel'd-Jimbo Hecke operator is defined as follows. Writing

$$
\mathcal{R}\left(x_{i} \otimes x_{j}\right)=\sum_{k, l} x_{k} \otimes x_{l} \mathcal{R}_{i, j}^{k, l}
$$

the matrix components $\mathcal{R}_{i, j}^{k, l} \in \mathbb{k}$ are given by

$$
\mathcal{R}_{i, j}^{k, l}=\frac{q^{2}-q^{2 \varepsilon_{i, j}}}{1+q^{2 \varepsilon_{i, j}}} \delta_{i, j}^{k, l}+(-1)^{\hat{i} \hat{j}} \frac{q^{\varepsilon_{i, j}}\left(q^{2}+1\right)}{1+q^{2 \varepsilon_{i, j}}} \delta_{i, j}^{l, k}
$$

Here, $\varepsilon_{i, j}=\operatorname{sgn}(i-j)$. Thus,

$$
\begin{array}{ll}
\mathcal{R}_{i i}^{i i}=q^{2} & \text { if } \hat{i}=\overline{0}, \\
\mathcal{R}_{i i}^{i i}=-1 & \text { if } \hat{i}=\overline{1}, \\
\mathcal{R}_{i j}^{i j}=q^{2}-1 & \text { if } i<j, \\
\mathcal{R}_{i j}^{j i}=(-1)^{\hat{i} \hat{j}} q & \text { if } i \neq j,
\end{array}
$$

and $\mathcal{R}_{i, j}^{k, l}=0$ in all other cases. One checks that $\mathcal{R}$ is a Hecke operator that is associated to $q^{2}$. 


\section{The supercharacter}

2.1. Superalgebras, supercoalgebras etc. An algebra $\mathcal{A}$ in $\operatorname{Vect}_{\mathbb{k}}^{S}$ is called a superalgebra over $\mathbb{k}$; this is just an ordinary $\mathbb{k}$-algebra such that the unit map $\mathbb{k} \rightarrow \mathcal{A}$ and the multiplication

$$
\mu: \mathcal{A} \otimes \mathcal{A} \rightarrow \mathcal{A}
$$

are morphisms in Vect $t_{\mathbb{k}}^{s}$. In other words, $\mathcal{A}$ is a $\mathbb{Z}_{2}$-graded $\mathbb{k}$-algebra in the usual sense: $\mathcal{A}=\mathcal{A}_{\overline{0}} \oplus \mathcal{A}_{\overline{1}}$ with $\mathbb{k}$-subspaces $\mathcal{A}_{\overline{0}}$ and $\mathcal{A}_{\overline{1}}$ such that $\mathcal{A}_{\bar{r}} \mathcal{A}_{\bar{s}} \subseteq \mathcal{A}_{\overline{r+s}}$. Homomorphisms of superalgebras, by definition, are algebra maps in $\operatorname{Vect}_{\mathbb{k}}^{s}$, that is, they preserve the $\mathbb{Z}_{2}$-grading.

If $V$ is a vector superspace in $\operatorname{Vect}_{\mathbb{k}}^{S}$ then the tensor algebra $\mathrm{T}(V)=\bigoplus_{n \geq 0} V^{\otimes n}$ is a superalgebra via the $\mathbb{Z}_{2}$-grading of each $V^{\otimes n}$ as in $\S 1.2$. In general, if $\mathcal{A}$ is any superalgebra, then by selecting a $\mathbb{Z}_{2}$-graded subspace $V \subseteq \mathcal{A}$ which generates the algebra $\mathcal{A}$, we obtain a canonical isomorphism of superalgebras

$$
\mathrm{T}(V) /(R) \stackrel{\sim}{\longrightarrow} \mathcal{A}
$$

where $(R)$ is the two-sided ideal of $\mathrm{T}(V)$ that is generated by a $\mathbb{Z}_{2}$-graded linear subspace $R \subseteq \mathrm{T}(V)$.

Given superalgebras $\mathcal{A}$ and $\mathcal{B}$, the tensor product $\mathcal{A} \otimes \mathcal{B}$ is the superalgebra with the usual additive structure and grading and with multiplication $\mu_{\mathcal{A} \otimes \mathcal{B}}$ defined by using the supersymmetry map (1.1): $\mu_{\mathcal{A} \otimes \mathcal{B}}=\left(\mu_{\mathcal{A}} \otimes \mu_{\mathcal{B}}\right) \circ\left(\operatorname{Id}_{A} \otimes c_{\mathcal{B}, \mathcal{A}} \otimes \operatorname{Id}_{\mathcal{B}}\right)$ or, explicitly,

$$
(a \otimes b)\left(a^{\prime} \otimes b^{\prime}\right)=(-1)^{\hat{a}^{\prime} \hat{b}} a a^{\prime} \otimes b b^{\prime}
$$

for homogeneous $a^{\prime} \in \mathcal{A}$ and $b \in \mathcal{B}$. In other words, the canonical images of $\mathcal{A}$ and $\mathcal{B}$ in $\mathcal{A} \otimes \mathcal{B}$ supercommute, in the sense that the supercommutator

$$
[a, b]=a b-(-1)^{\hat{a} \hat{b}} b a
$$

vanishes for any pair of homogeneous elements $a \in \mathcal{A}$ and $b \in \mathcal{B}$.

Supercoalgebras, superbialgebras etc. are defined similarly as suitable objects of $\operatorname{Vect}_{\mathbb{k}}^{s}$ such that all structure maps are maps in $\operatorname{Vect}_{\mathbb{k}}^{s}$. The compatibility between the comultiplication $\Delta$ and the multiplication of a superbialgebra $\mathcal{B}$ amounts to the following rule:

$$
\Delta(a b)=\sum_{(a),(b)}(-1)^{\hat{a}_{(2)} \hat{b}_{(1)}} a_{(1)} b_{(1)} \otimes a_{(2)} b_{(2)}
$$

for homogeneous elements $a, b \in \mathcal{B}$. Here we use the Sweedler notation $\Delta(a)=$ $\sum_{(a)} a_{(1)} \otimes a_{(2)}$ and $a_{(1)}, a_{(2)}$ are chosen homogeneous with $\hat{a}_{(1)}+\hat{a}_{(2)}=\hat{a}$. 
Example 2.1 (Symmetric superalgebra [40, 3.2.5]). The symmetric superalgebra of a given $V$ in $\operatorname{Vect}_{\mathfrak{k}}^{s}$ is defined by

$$
\mathrm{S}(V)=\mathrm{T}(V) /\left([v, w]_{\otimes} \mid v, w \in V\right)
$$

where $[v, w]_{\otimes}$ is the supercommutator (2.2) in $\mathbf{T}(V)$. Ignoring parity, $\mathbf{S}(V)$ is isomorphic to $S\left(V_{\overline{0}}\right) \otimes \Lambda\left(V_{\overline{1}}\right)$, where $S($. $)$ and $\Lambda($. $)$ denote the ordinary symmetric and exterior (Grassmann) algebras, respectively. The symmetric superalgebra is a Hopf superalgebra: comultiplication $\Delta: \mathrm{S}(V) \rightarrow \mathrm{S}(V) \otimes \mathrm{S}(V)$ is given by $\Delta(v)=v \otimes 1+1 \otimes v$ for $v \in V$ and extension to all of $\mathrm{S}(V)$ by multiplicativity. Similarly, the counit $\varepsilon: \mathrm{S}(V) \rightarrow \mathbb{k}$ is given by $\varepsilon(v)=0$ and the antipode $\mathcal{S}: \mathbf{S}(V) \rightarrow \mathrm{S}(V)$ by $\mathcal{S}(v)=-v$ for $v \in V$.

2.2. Comodules. We refer to [29], Chap. III, for background on comodules, comodule algebras etc.

Given a superbialgebra $\mathcal{B}$, we let $\operatorname{Comod}_{\mathcal{B}}^{S}$ denote the category of all right $\mathcal{B}$ comodules and $\mathcal{B}$-comodule maps in $\operatorname{Vect}_{\mathfrak{k}}^{s}$. Thus, for any object $V$ in $\operatorname{Comod}_{\mathcal{B}}^{s}$, we have a "coaction" morphism

$$
\delta_{V}: V \rightarrow V \otimes \mathcal{B}
$$

in Vect $t_{\mathbb{k}}^{S}$. If $x_{1}, \ldots, x_{d}$ is a fixed basis of $V$ consisting of homogeneous elements, with $\hat{i}$ denoting the parity of $x_{i}$ as before, then we will write

$$
\delta_{V}\left(x_{j}\right)=\sum_{i} x_{i} \otimes b_{j}^{i} \quad \text { with } b_{j}^{i} \in B_{\hat{i}+\hat{j}} .
$$

The tensor product of vector superspaces makes $\operatorname{Comod}_{\mathcal{B}}^{s}$ into a tensor category: if $U$ and $V$ are in $\operatorname{Comod}_{\mathcal{B}}^{S}$ then $\mathcal{B}$ coacts on $U \otimes V$ by

$$
\delta_{U \otimes V}: U \otimes V \stackrel{\delta_{U} \otimes \delta_{V}}{\longrightarrow} U \otimes \mathcal{B} \otimes V \otimes \mathcal{B} \stackrel{c_{B, V}}{\longrightarrow} U \otimes V \otimes \mathcal{B} \otimes \mathcal{B} \stackrel{\operatorname{Id} \otimes \mu_{\mathcal{B}}}{\longrightarrow} U \otimes V \otimes \mathcal{B} .
$$

If $\mathcal{B}$ is supercommutative as a superalgebra then the supersymmetry $c_{U, V}$ is a $\mathcal{B}$ comodule morphism, i.e., $\delta_{V \otimes U} \circ c_{U, V}=\left(c_{U, V} \otimes \operatorname{Id}_{\mathcal{B}}\right) \circ \delta_{U \otimes V}$. Therefore $\operatorname{Comod}_{\mathcal{B}}^{S}$ is a symmetric tensor category in this case.

2.3. The supercharacter map. Let $\mathcal{B}$ denote a superbialgebra and let $V$ be a finite dimensional object in $\operatorname{Comod}_{\mathcal{B}}^{S}$. The coaction $\delta_{V}$ is an even map in $\operatorname{Hom}_{\mathfrak{k}}(V, V \otimes \mathcal{B})$. Consider the following morphism in $\operatorname{Vect}_{\mathbb{k}}^{S}$ :

$$
\chi^{s}: \operatorname{End}_{\mathbb{k}}(V) \stackrel{\delta_{V} \circ(\cdot)}{\longrightarrow} \operatorname{Hom}_{\mathbb{k}}(V, V \otimes \mathcal{B}) \underset{(1.6)}{\sim} \operatorname{End}_{\mathfrak{k}}(V) \otimes \mathcal{B} \stackrel{\text { str } \otimes \mathrm{Id}}{\longrightarrow} \mathbb{k} \otimes \mathcal{B}=\mathcal{B}
$$

where str is the supertrace as in (1.8). This map will be called the supercharacter map of $V$. Forgetting parity and viewing all elements as even, the supertrace becomes 
the ordinary trace and the supercharacter becomes the usual character. These will be denoted by tr and $\chi$, respectively.

In particular, we have the element

$$
\chi_{V}^{s}:=\chi^{s}\left(\operatorname{Id}_{V}\right) \in \mathcal{B}_{\overline{0}} .
$$

To obtain explicit formulas, fix a basis $x_{1}, \ldots, x_{d}$ of $V$ consisting of homogeneous elements and let $\left(F_{j}^{i}\right)$ and $\left(b_{j}^{i}\right)$ be the matrices of $f \in \operatorname{End}_{\mathfrak{k}}(V)$ and of $\delta_{V}$ with respect to this basis as in (1.2), (2.3). Then

$$
\chi^{s}(f)=\sum_{i, j}(-1)^{\hat{i} \hat{j}} b_{j}^{i} F_{i}^{j} .
$$

Let $\varepsilon: \mathcal{B} \rightarrow \mathbb{k}$ denote the counit of $\mathcal{B}$. Then $x_{j}=\sum_{i} x_{i} \varepsilon\left(b_{j}^{i}\right)$ holds in (2.3). Hence $\varepsilon\left(b_{j}^{i}\right)=\delta_{j}^{i} \cdot 1_{\mathbb{k}}$ and (2.6) gives

$$
\varepsilon\left(\chi^{s}(f)\right)=\operatorname{str}(f) .
$$

When $f$ is even formula (2.6) becomes $\chi^{s}(f)=\sum_{i, j}(-1)^{\hat{i}} b_{j}^{i} F_{i}^{j}$ since $F_{i}^{j}=0$ unless $\hat{i}+\hat{j}=\overline{0}$. In particular,

$$
\chi_{V}^{s}=\sum_{i}(-1)^{\hat{i}} b_{i}^{i} .
$$

In the following, we let comod ${ }_{\mathcal{B}}^{s}$ denote the full subcategory of $\operatorname{Comod}_{\mathcal{B}}^{s}$ consisting of all objects that are finite-dimensional over $k$. The supercharacter has the following properties analogous to standard properties of the ordinary character.

Lemma 2.2. Let $\mathcal{B}$ denote a superbialgebra and let $U, V$ and $W$ be objects of $\operatorname{comod}_{\mathcal{B}}^{S}$.

(a) If $f: V \rightarrow U$ and $g: U \rightarrow V$ are $\mathcal{B}$-comodule maps (not necessarily even) then

$$
\chi^{s}(f \circ g)=(-1)^{\hat{f} \hat{s}} \chi^{s}(g \circ f) .
$$

(b) For $f \in \operatorname{End}_{\mathbb{k}}(V), g \in \operatorname{End}_{\mathfrak{k}}(U)$ view $f \otimes g \in \operatorname{End}_{\mathbb{k}}(V \otimes U)$ as in (1.7). Then

$$
\chi^{s}(f \otimes g)=\chi^{s}(f) \chi^{s}(g) .
$$

(c) Given an exact sequence $0 \rightarrow U \stackrel{\mu}{\rightarrow} V \rightarrow W \rightarrow 0$ in $\operatorname{comod}_{\mathcal{B}}^{s}$, let $f \in \operatorname{End}_{\mathbb{k}}(V)$ be such that $f(\mu(U)) \subseteq \mu(U)$, and let $g \in \operatorname{End}_{\mathbb{k}}(U), h \in \operatorname{End}_{\mathbb{k}}(W)$ be the maps induced by $f$. Then

$$
\chi^{s}(f)=\chi^{s}(g)+\chi^{s}(h) .
$$

In particular, $\chi_{V}^{s}=\chi_{U}^{s}+\chi_{W}^{s}$. Moreover, if $f \in \operatorname{End}_{\operatorname{comod}_{\mathcal{B}}^{s}}(V)$ is a projection (i.e., $f^{2}=f$ ) then $\chi^{s}(f)=\chi_{\operatorname{Im} f}^{s}$. 
Proof. (a) Let $T_{V}$ denote the map $\operatorname{Hom}_{\mathbb{k}}(V, V \otimes \mathcal{B}) \rightarrow \mathcal{B}$ in (2.5); so $\chi^{s}(f)=$ $T_{V}\left(\delta_{V} \circ f\right)$. Since $f$ and $g$ are comodule maps, we have $\delta_{U} \circ f=\left(f \otimes \operatorname{Id}_{\mathcal{B}}\right) \circ \delta_{V}$ and similarly for $g$. Putting $h=\delta_{U} \circ f \in \operatorname{Hom}_{\mathbb{k}}(V, U \otimes \mathcal{B})$ we obtain $\chi^{s}(f \circ g)=$ $T_{U}\left(\delta_{U} \circ f \circ g\right)=T_{U}(h \circ g)$ and $\chi^{s}(g \circ f)=T_{V}\left(\delta_{V} \circ g \circ f\right)=T_{V}\left(\left(g \otimes \operatorname{Id}_{\mathcal{B}}\right) \circ h\right)$. Therefore, we must show that

$$
T_{U}(h \circ g)=(-1)^{\hat{f} \hat{g}} T_{V}\left(\left(g \otimes \operatorname{Id}_{\mathcal{B}}\right) \circ h\right) .
$$

Using the identification $\operatorname{Hom}_{\mathbb{k}}(V, U \otimes \mathcal{B}) \cong \operatorname{Hom}_{\mathbb{k}}(V, U) \otimes \mathcal{B}$ as in (1.6), write $h=\sum_{i} f_{i} \otimes b_{i}$ with $f_{i} \in \operatorname{Hom}_{\mathbb{k}}(V, U), b_{i} \in \mathcal{B}$, and $\hat{f}_{i}+\hat{b}_{i}=\hat{h}=\hat{f}$. Then $h \circ g \in \operatorname{Hom}_{\mathbb{k}}(U, U \otimes \mathcal{B})$ becomes the element $\left(\sum_{i} f_{i} \otimes b_{i}\right) \circ g=$ $\sum_{i}(-1)^{\hat{b_{i}} \hat{g}}\left(f_{i} \circ g\right) \otimes b_{i} \in \operatorname{End}_{\mathbb{k}}(U) \otimes \mathcal{B}$, and $\left(g \otimes \operatorname{Id}_{\mathcal{B}}\right) \circ h \operatorname{Hom}_{\mathbb{k}}(V, V \otimes \mathcal{B})$ becomes $\sum_{i}\left(g \circ f_{i}\right) \otimes b_{i}$. The standard identity $\operatorname{str}\left(f_{i} \circ g\right)=(-1)^{\hat{f}_{i} \hat{g}} \operatorname{str}\left(g \circ f_{i}\right)$ (cf., e.g., [40], §3 (b), p. 165), now yields

$$
\begin{aligned}
T_{U}(h \circ g) & =\sum_{i}(-1)^{\hat{b}_{i} \hat{g}} \operatorname{str}\left(f_{i} \circ g\right) \otimes b_{i} \\
& =\sum_{i}(-1)^{\hat{b_{i}} \hat{g}+\hat{f}_{i} \hat{g}} \operatorname{str}\left(g \circ f_{i}\right) \otimes b_{i} \\
& =(-1)^{\hat{f} \hat{g}} T_{V}\left(\left(g \otimes \operatorname{Id}_{\mathcal{B}}\right) \circ h\right)
\end{aligned}
$$

as desired.

(b) Fix homogeneous $\mathbb{k}$-bases $\left\{x_{i}\right\}$ and $\left\{y_{\ell}\right\}$ of $V$ and $U$, respectively, and write $\hat{x}_{i}=\hat{i}, \hat{y}_{\ell}=\hat{\ell}$ as usual. Moreover, let $\left(F_{j}^{i}\right)$ and $\left(G_{m}^{\ell}\right)$ be the matrices of $f$ and $g$ for these bases, as in (1.2). Then $\left\{x_{i} \otimes y_{\ell}\right\}$ is a basis of $V \otimes U$, with $x_{i} \otimes y_{\ell}$ having parity $\hat{i}+\hat{\ell}$. Moreover,

$$
\begin{aligned}
(f \otimes g)\left(x_{j} \otimes y_{m}\right) & =(-1)^{\hat{g} \hat{j}} f\left(x_{j}\right) \otimes g\left(y_{m}\right) \\
& =(-1)^{\hat{g} \hat{j}} \sum_{i} x_{i} F_{j}^{i} \otimes \sum_{\ell} y_{\ell} G_{m}^{\ell} \\
& =\sum_{i, \ell} x_{i} \otimes y_{\ell} \Phi_{j, m}^{i, \ell} \quad \text { with } \Phi_{j, m}^{i, \ell}=(-1)^{(\hat{\ell}+\hat{m}) \hat{j}} F_{j}^{i} G_{m}^{\ell}
\end{aligned}
$$

because $G_{\ell}^{m}=0$ unless $\hat{\ell}+\hat{m}=\hat{g}$. Similarly, writing $\delta_{V}\left(x_{j}\right)=\sum_{i} x_{i} \otimes b_{j}^{i}$ with $b_{j}^{i} \in \mathcal{B}_{\hat{i}+\hat{j}}$ and $\delta_{U}\left(y_{m}\right)=\sum_{\ell} y_{\ell} \otimes c_{m}^{\ell}$ with $c_{m}^{\ell} \in \mathcal{B}_{\hat{\ell}+\hat{m}}$, one obtains using (2.4)

$$
\delta_{V \otimes U}\left(x_{j} \otimes y_{m}\right)=\sum_{i, \ell} x_{i} \otimes y_{\ell} \otimes \Psi_{j, m}^{i, \ell} \quad \text { with } \Psi_{j, m}^{i, \ell}=(-1)^{(\hat{i}+\hat{j}) \hat{\ell}} b_{j}^{i} c_{m}^{\ell} .
$$


Therefore, formula (2.6) becomes

$$
\begin{aligned}
\chi^{s}(f \otimes g) & =\sum_{i, \ell, j, m}(-1)^{(\hat{i}+\hat{\ell})(\hat{j}+\hat{m})} \Psi_{j, m}^{i, \ell} \Phi_{i, \ell}^{j, m} \\
& =\sum_{i, \ell, j, m}(-1)^{\hat{i} \hat{j}+\hat{\ell} \hat{m}} b_{j}^{i} F_{i}^{j} c_{m}^{\ell} G_{\ell}^{m} \\
& =\chi^{s}(f) \chi^{s}(g) .
\end{aligned}
$$

(c) Choose a basis $\left\{x_{i}\right\}$ of $V$ consisting of homogeneous elements so that $x_{i}=$ $\mu\left(y_{i}\right)$ for $i \leq \operatorname{dim} U$ and let $\left(F_{j}^{i}\right)$ be the matrix of $f$ for this basis. Then $F_{j}^{i}=0$ for $i>\operatorname{dim} U, j \leq \operatorname{dim} U$. Moreover, the $y_{i}$ form a basis of $U$ and the $z_{i}=\pi\left(x_{i}\right)$ form a basis of $W$, and the matrices of $g$ and $h$ for these bases are $\left(F_{j}^{i}\right)_{i, j \leq \operatorname{dim} U}$ and $\left(F_{j}^{i}\right)_{i, j>\operatorname{dim} U}$, respectively. Similarly, if $\left(b_{j}^{i}\right)$ is the matrix of $\delta_{V}$ with respect to the basis $\left\{x_{i}\right\}$ as in (2.3) then $b_{j}^{i}=0$ for $i>\operatorname{dim} U, j \leq \operatorname{dim} U$, and the matrices of $\delta_{U}$ and $\delta_{W}$ for the given bases are $\left(b_{j}^{i}\right)_{i, j \leq \operatorname{dim} U}$ and $\left(b_{j}^{i}\right)_{i, j>\operatorname{dim} U}$, respectively. Therefore,

$$
\begin{aligned}
\chi^{s}(f) & =\sum_{i, j}(-1)^{\hat{i} \hat{j}} b_{j}^{i} F_{i}^{j} \\
& =\sum_{i, j \leq \operatorname{dim} U}(-1)^{\hat{i} \hat{j}} b_{j}^{i} F_{i}^{j}+\sum_{i, j>\operatorname{dim} U}(-1)^{\hat{i} \hat{j}} b_{j}^{i} F_{i}^{j} \\
& =\chi^{s}(g)+\chi^{s}(h) .
\end{aligned}
$$

The remaining assertions are clear.

2.4. The Grothendieck ring. Let $\mathcal{B}$ be a superbialgebra and let

$$
R_{\mathcal{B}}=K_{0}\left(\operatorname{comod}_{\mathcal{B}}^{S}\right)
$$

denote the Grothendieck group of the category comod $\operatorname{com}_{\mathcal{B}}^{S}$. Thus, for each $V$ in $\operatorname{comod}_{\mathcal{B}}^{S}$, there is an element $[V] \in R_{\mathcal{B}}$ and each short exact sequence $0 \rightarrow U \rightarrow V \rightarrow W \rightarrow 0$ in $\operatorname{comod}_{\mathcal{B}}^{S}$ gives rise to an equation $[V]=[U]+[W]$ in $R_{\mathcal{B}}$. The group $R_{\mathcal{B}}$ is in fact a ring with multiplication given by the tensor product of $\mathcal{B}$-comodules. If $\mathcal{B}$ is supercommutative as a superalgebra then the ring $R_{\mathcal{B}}$ is commutative; see $\S 2.2$.

Both the ordinary dimension and the superdimension are additive on short exact sequences and multiplicative on tensor products. Hence they yield ring homomorphisms

$$
\operatorname{dim}, \operatorname{sdim}: R_{\mathcal{B}} \rightarrow \mathbb{Z} \text {. }
$$

Parts (b) and (c) of Lemma 2.2 and formula (2.7) have the following immediate consequence: 
Corollary 2.3. The map $[V] \mapsto \chi_{V}^{s}$ yields a well-defined ring homomorphism $\chi^{s}: R_{\mathcal{B}} \rightarrow \mathcal{B}_{\overline{0}}$. Furthermore, the following diagram commutes:

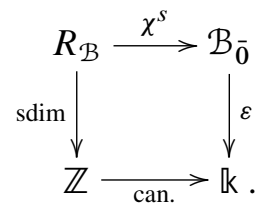

Forgetting the $\mathbb{Z}_{2}$-grading, the corollary also gives the more familiar version with $\chi$ and $\operatorname{dim}$ in place of $\chi^{s}$ and sdim, respectively.

2.5. General linear supergroup and Berezinian. Let $V$ in Vect $t_{\mathbb{k}}^{s}$ be finite-dimensional and fix a standard basis $x_{1}, \ldots, x_{d}$ with $\hat{i}=\overline{0}(i \leq p)$ and $\hat{i}=\overline{1}(i>p)$.

2.5.1. For each supercommutative $\mathbb{k}$-superalgebra $\mathcal{R}$ we denote by $\mathrm{E}(V)(\mathcal{R})$ the set of all $\mathcal{R}$-linear maps $V \otimes \mathcal{R} \rightarrow V \otimes \mathcal{R}$ in $\operatorname{Vect}_{\mathfrak{k}}^{s}$. Using the identification $\operatorname{End}_{\mathcal{R}}(V \otimes \mathcal{R}) \cong$ $\operatorname{Hom}_{\mathbb{k}}(V, V \otimes \mathcal{R}) \cong \operatorname{End}_{\mathbb{k}}(V) \otimes \mathcal{R}$ (see (1.6)), we may view $\mathrm{E}(V)(\mathcal{R})$ as the even subspace of $\operatorname{End}_{\mathfrak{k}}(V) \otimes \mathcal{R}$ :

$$
\mathrm{E}(V)(\mathcal{R})=\left(\operatorname{End}_{\mathbb{k}}(V) \otimes \mathcal{R}\right)_{\overline{0}} .
$$

This defines a functor $\mathrm{E}(V)$ from the category of supercommutative $k$-superalgebras to the category of semigroups.

2.5.2. Tensoring the supertrace $\operatorname{str}: \operatorname{End}_{\mathbb{k}}(V) \rightarrow \mathbb{k}$ of (1.8) with $\operatorname{Id}_{\mathcal{R}}$, we obtain an $\mathcal{R}$-linear supertrace map str: $\operatorname{End}_{\mathbb{k}}(V) \otimes \mathcal{R} \rightarrow \mathcal{R}$ in $\operatorname{Vect}_{\mathbb{k}}^{S}$ which restricts to a map $\mathrm{E}(V)(\mathcal{R}) \rightarrow \mathcal{R}_{\overline{0}}$. The given standard basis $x_{1}, \ldots, x_{d}$ of $V$ is an $\mathcal{R}$-basis of $V \otimes \mathcal{R}$. In terms of this basis, an element $\phi \in \mathrm{E}(V)(\mathcal{R})$ is given by

$$
\phi\left(x_{j}\right)=\sum_{i=1}^{d} x_{i} \Phi_{j}^{i} \quad \text { with } \Phi_{j}^{i} \in \mathcal{R}_{\hat{i}+\hat{j}} .
$$

Thus $\phi$ is described by a supermatrix $\Phi=\left(\Phi_{j}^{i}\right)$ in standard form over $\mathcal{R}$ :

$$
\Phi=\left(\begin{array}{ll}
A & B \\
C & D
\end{array}\right)
$$

where $A=\left(\Phi_{j}^{i}\right)_{i, j \leq p}$ and $D=\left(\Phi_{j}^{i}\right)_{i, j>p}$ are square matrices with entries in $\mathcal{R}_{\overline{0}}$ while $C, D$ are matrices over $\mathcal{R}_{\overline{1}}$. The supertrace of $\phi$ is given by

$$
\operatorname{str}(\phi)=\sum_{i}(-1)^{\hat{i}} \Phi_{i}^{i}=\operatorname{tr}(A)-\operatorname{tr}(D)=: \operatorname{str}(\Phi) .
$$


2.5.3. The functor $\mathrm{E}(V)$ is represented by a supercommutative $\mathbb{k}$-superbialgebra which coacts on $V$; this algebra will be denoted by

$$
\mathcal{B}=\mathcal{O}(\mathrm{E}(V)) \text {. }
$$

Thus, there is a natural isomorphism of $\mathrm{E}(V)$ with the functor $\operatorname{Hom}(\mathcal{B}$, ?) of parity preserving algebra homomorphisms. In particular, the identity map on $\mathcal{B}$ corresponds to an element $\xi \in \mathrm{E}(V)(\mathcal{B})$. Let $X=\left(x_{j}^{i}\right)_{d \times d}$ be the matrix of $\xi$, as in (2.9). The elements $x_{j}^{i}$ have parity $\hat{i}+\hat{j}$ and they form a set of supercommuting algebraically independent generators of $\mathcal{B}$. In fact, $\mathcal{B}$ is isomorphic to the symmetric superalgebra $\mathrm{S}\left(V^{*} \otimes V\right)$, with $x_{j}^{i} \mapsto x^{i} \otimes x_{j}$, where $\left\{x^{i}\right\} \subseteq V^{*}$ is the dual basis for the given basis of $V$.

We can think of $X$ as the generic supermatrix with respect to the given basis of $V$ : any supermatrix $\Phi=\left(\Phi_{j}^{i}\right)$ as in (2.9) comes from an algebra map $\mathcal{B} \rightarrow \mathcal{R}$ via $x_{j}^{i} \mapsto \Phi_{j}^{i}$. The canonical coaction $\delta: V \rightarrow V \otimes \mathcal{B}$, the comultiplication $\Delta$ and the counit $\varepsilon$ of $\mathcal{B}$ are given by

$$
\begin{aligned}
\delta\left(x_{j}\right) & =\sum_{i} x_{i} \otimes x_{j}^{i}, \\
\Delta\left(x_{j}^{i}\right) & =\sum_{k} x_{k}^{i} \otimes x_{j}^{k}, \\
\varepsilon\left(x_{j}^{i}\right) & =\delta_{j}^{i} .
\end{aligned}
$$

These formulas can also be written as $\delta\left(x_{1}, \ldots, x_{d}\right)=\left(x_{1}, \ldots, x_{d}\right) \otimes X, \Delta(X)=$ $X \otimes X$ and $\varepsilon(X)=1$.

2.5.4. Similarly, $\mathrm{GL}(V)(\mathcal{R})$ is defined, for any supercommutative $\mathbb{k}$-superalgebra $\mathcal{R}$, as the set of all invertible $\mathcal{R}$-linear endomorphism of $V \otimes \mathcal{R}$ in $\operatorname{Vect}_{\mathbb{k}}^{S}$. The condition for a supermatrix $\Phi$ in standard form (as in (2.10)) to be invertible is that $A$ and $D$ are invertible as ordinary matrices over $\mathcal{R}_{\overline{0}}$. In this case, the inverse of $\Phi$ is given by

$$
\Phi^{-1}=\left(\begin{array}{cc}
\left(A-B D^{-1} C\right)^{-1} & -A^{-1} B\left(D-C A^{-1} B\right)^{-1} \\
-D^{-1} C\left(A-B D^{-1} C\right)^{-1} & \left(D-C A^{-1} B\right)^{-1}
\end{array}\right) .
$$

See Berezin [3], Theorem 3.1 and Lemma 3.2. The element

$$
\operatorname{ber}(\Phi):=\operatorname{det}(A) \operatorname{det}\left(D-C A^{-1} B\right)^{-1}=\operatorname{det}(D)^{-1} \operatorname{det}\left(A-B D^{-1} C\right)
$$

is called the superdeterminant or Berezinian of $\Phi$; it is an invertible element of $\mathcal{R}_{\overline{0}}$.

The functor $\mathrm{GL}(V)$ is represented by a supercommutative Hopf superalgebra $\mathcal{O}(\mathrm{GL}(V))$ which is generated over $\mathcal{B}=\mathcal{O}(\mathrm{E}(V))$ by $\operatorname{det}\left(X_{11}\right)^{-1}$ and $\operatorname{det}\left(X_{22}\right)^{-1}$, where $X_{11}=\left(x_{j}^{i}\right)_{i, j \leq p}$ and $X_{22}=\left(x_{j}^{i}\right)_{i, j>p}$ are the even blocks of the generic supermatrix $X$. By [3], Theorem 3.3, the $\operatorname{Berezinian} \operatorname{ber}(X)$ is a group-like element in $\mathcal{O}(\mathrm{GL}(V))$. 
2.6. Supersymmetric functions and exterior powers. Throughout this section, $V$ will denote a finite-dimensional vector superspace over $\mathbb{k}$. We assume that the characteristic of $\mathbb{k}$ is zero.

2.6.1. Let

$$
Y_{n}=\frac{1}{n !} \sum_{\sigma \in \mathbb{S}_{n}} \operatorname{sgn}(\sigma) \sigma \in \mathbb{k}\left[\Im_{n}\right]
$$

be the antisymmetrizer idempotent of the group algebra $\mathbb{k}\left[\subseteq_{n}\right]$ and define

$$
\wedge^{n} V:=\operatorname{Im} c_{Y_{n}} \subseteq V^{\otimes n}
$$

where $c: \mathbb{k}\left[\Im_{n}\right] \rightarrow \operatorname{End}_{\mathrm{Vect}_{\mathrm{k}}^{s}}\left(V^{\otimes n}\right)$ is as in (1.10). Thus, $\Lambda^{n} V$ is the space of antisymmetric $n$-tensors,

$$
\Lambda^{n} V=\left\{y \in V^{\otimes n} \mid c_{\sigma}(y)=\operatorname{sgn}(\sigma) y \text { for all } \sigma \in \widetilde{\Im}_{n}\right\} .
$$

For later use, we describe an explicit basis of $\Lambda^{n} V$. To this end, fix a standard basis $x_{1}, \ldots, x_{d}$ of $V$, with $\hat{i}=\overline{0}$ for $i \leq p$ and $\hat{i}=\overline{1}$ for $i>p$. Then the products $x_{\boldsymbol{i}}=x_{i_{1}} \otimes x_{i_{2}} \otimes \cdots \otimes x_{i_{n}}$ for sequences $\boldsymbol{i}=\left(i_{1}, i_{2}, \ldots, i_{n}\right) \in\{1,2, \ldots, d\}^{n}$ form a graded basis of $V^{\otimes n}$ that is permuted up to a \pm -sign by the action of $\subseteq_{n}$ on $V^{\otimes n}$; see formula (1.9):

$$
c_{\sigma}\left(x_{i}\right)=\operatorname{sgn}_{\boldsymbol{i}}(\sigma) x_{\sigma(\boldsymbol{i})}
$$

with

$$
\operatorname{sgn}_{\boldsymbol{i}}(\sigma)=(-1)^{\sum_{(p, q) \in \operatorname{inv}(\sigma)} \hat{i_{p}} \hat{i_{q}}} \text { and } \sigma(\boldsymbol{i})=\left(i_{\sigma^{-1}(1)}, i_{\sigma^{-1}(2)}, \ldots, i_{\sigma^{-1}(n)}\right) .
$$

Therefore, by elementary properties of monomial group representations, a $\mathbb{k}$-basis of $\Lambda^{n} V$ is given by the nonzero elements $c_{Y_{n}}\left(x_{i}\right)$ where $\boldsymbol{i}$ ranges over a transversal for the $\widetilde{S}_{n}$-action on $\{1,2, \ldots, d\}^{n}$. Such a transversal is provided by the weakly increasing sequences $\boldsymbol{i} \in\{1,2, \ldots, d\}^{n}$. Moreover, for a weakly increasing $\boldsymbol{i}$, it is easily seen from (2.14) that $c_{Y_{n}}\left(x_{i}\right)=0$ holds precisely if $i_{\ell}=i_{\ell+1} \leq p$ for some $\ell$. Therefore, a basis of $\wedge^{n} V$ is given by the elements $c_{Y_{n}}\left(x_{\boldsymbol{i}}\right)$ with $\boldsymbol{i}=\left(i_{1}<i_{2}<\right.$ $\left.\cdots<i_{m}<i_{m+1} \leq \cdots \leq i_{n}\right) \in\{1,2, \ldots, d\}^{n}$ and $i_{m} \leq p<i_{m+1}$.

In particular,

$$
\operatorname{dim}_{\mathbb{k}} \Lambda^{n} V=\sum_{m+m^{\prime}=n}\left(\begin{array}{c}
p \\
m
\end{array}\right)\left(\begin{array}{c}
q+m^{\prime}-1 \\
m^{\prime}
\end{array}\right)
$$

where $p=\operatorname{dim}_{\mathbb{k}} V_{\overline{0}}$ and $q=\operatorname{dim}_{\mathbb{k}} V_{\overline{1}}$. Equivalently, the generating power series in $\mathbb{Z} \llbracket t \rrbracket$ for the sequence $\operatorname{dim}_{\mathbb{k}} \Lambda^{n} V$ is given by

$$
\sum_{n \geq 0} \operatorname{dim}_{\mathbb{k}} \Lambda^{n} V t^{n}=\frac{(1+t)^{p}}{(1-t)^{q}} .
$$

When $q>0$ then all $\wedge^{n} V$ are nonzero. For additional details on exterior powers, see, e.g., [43], Sections I.5 and I.7. 
2.6.2. Consider the superbialgebra $\mathcal{B}=\mathcal{O}(E(V))$ as defined in $\S 2.5 .3$ and recall that $V$ is in $\operatorname{comod}_{\mathcal{B}}^{S}$. The representation $c: \mathbb{k}\left[\widetilde{S}_{n}\right] \rightarrow \operatorname{End}_{\operatorname{Vect}_{\mathbb{k}}^{s}}\left(V^{\otimes n}\right)$ of (1.10) actually has image in $\operatorname{End}_{\text {comod }_{\mathcal{B}}^{s}}\left(V^{\otimes n}\right)$, since $\mathcal{B}$ is supercommutative. Therefore, $\Lambda^{n} V$ also belongs to $\operatorname{comod}_{\mathcal{B}}^{S}$ and we can define the $n^{\text {th }}$ elementary supersymmetric function by

$$
e_{n}:=\chi_{\Lambda^{n} V}^{s}=\chi^{s}\left(c_{Y_{n}}\right) \in \mathcal{B}_{\overline{0}} .
$$

Here, the equality $\chi_{\Lambda^{n} V}^{s}=\chi^{s}\left(c_{Y_{n}}\right)$ holds by Lemma 2.2 (c).

Similarly, one defines the $n^{\text {th }}$ super power sum by

$$
p_{n}:=\chi^{s}\left(c_{(1,2, \ldots, n)}\right) \in \mathcal{B}_{\overline{0}}
$$

where $(1,2, \ldots, n) \in \widetilde{S}_{n}$ the cyclic permutation mapping $1 \mapsto 2 \mapsto 3 \mapsto \ldots \mapsto$ $n \mapsto 1$. In terms of the generic supermatrix $X$ from $\$ 2.5 .3$, one has

$$
p_{n}=\operatorname{str}\left(X^{n}\right) .
$$

Modulo the space spanned by the Lie commutators $f g-g f$ with $f, g \in \mathbb{k}\left[\Im_{n}\right]$, the following relation is easily seen to hold in $\mathbb{k}\left[\widetilde{S}_{n}\right]$ :

$$
n Y_{n} \equiv \sum_{i=1}^{n}(-1)^{i-1}(1,2, \ldots, i) Y_{n-i}
$$

(with $Y_{0}=1$ ). Applying the function $\chi^{s} \circ c: \mathbb{k}\left[\widetilde{S}_{n}\right] \rightarrow \mathcal{B}_{\overline{0}}$ to this relation and using Lemma 2.2 (a), (b), one obtains the Newton relations:

$$
n e_{n}=\sum_{i=1}^{n}(-1)^{i-1} p_{i} e_{n-i} .
$$

Let $t$ be a formal parameter (of parity $\overline{0}$ ) and consider the generating functions $P(t)=$ $\sum_{n \geq 1} p_{n} t^{n-1}$ and $E(t)=\sum_{n \geq 0} e_{n} t^{n}$ in $\mathcal{B}_{\overline{0}} \llbracket t \rrbracket$. The Newton relations can be written in the form $P(-t)=\frac{d}{d t} \log E(t)$; see, e.g., [34], p. 23. Combining this with the identity

$$
\operatorname{ber}(\exp (t X))=\exp (\operatorname{str}(t X))
$$

due to Berezin ([3], Chapter 3, or [40], p. 167) one obtains the following expansion for the characteristic function ber $(1+t X)$ of the generic supermatrix $X$ :

Proposition 2.4. $\operatorname{ber}(1+t X)=\sum_{n \geq 0} e_{n} t^{n}$.

This proposition is known; see, e.g., Khudaverdian and Voronov [30], Prop. 1. 


\section{Homogeneous superalgebras}

3.1. $N$-homogeneous superalgebras. Let $N$ be an integer with $N \geq 2$. A homogeneous superalgebra of degree $N$ or $N$-homogeneous superalgebra is an algebra $\mathcal{A}$ of the form (2.1) with $V$ finite-dimensional and $R \subseteq V^{\otimes N}$ :

$$
\mathcal{A}=A(V, R) \cong \mathrm{T}(V) /(R) .
$$

The assumption $R \subseteq V^{\otimes N}$ implies that, besides the usual $\mathbb{Z}_{2}$-grading ("parity"), $\mathcal{A}$ also has a connected $\mathbb{Z}_{+}$-grading ("degree"),

$$
\mathcal{A}=\bigoplus_{n \geq 0} \mathcal{A}_{n}
$$

The algebra $\mathcal{A}$ is generated by $\mathcal{A}_{1}=V$ and all homogeneous components $\mathcal{A}_{n}$ are finite-dimensional objects of $\operatorname{Vect}_{\mathbb{k}}^{S}$. In fact,

$$
\mathcal{A}_{n} \cong V^{\otimes n} / R_{n} \quad \text { with } R_{n}:=(R) \cap V^{\otimes n}=\sum_{i+j+N=n} V^{\otimes i} \otimes R \otimes V^{\otimes j} .
$$

Note that $R_{n}=0$ for $n<N$; so $\mathcal{A}_{n} \cong V^{\otimes n}$ if $n<N$.

Morphisms of $N$-homogeneous superalgebras $f: \mathcal{A}=A(V, R) \rightarrow \mathcal{A}^{\prime}=$ $A\left(V^{\prime}, R^{\prime}\right)$ are morphism of superalgebras which also respect the $\mathbb{Z}_{+}$-grading. Equivalently, by restricting to degree 1 , we have a morphism $f_{1}: \mathcal{A}_{1}=V \rightarrow \mathcal{A}_{1}^{\prime}=V^{\prime}$ in Vect ${ }_{\mathbb{k}}^{s}$ whose $N^{\text {th }}$ tensor power satisfies $f_{1}^{\otimes N}(R) \subseteq R^{\prime}$. Thus, one has a category $\mathrm{H}_{N} \mathrm{Alg}_{\mathbb{k}}^{s}$ of $N$-homogeneous $\mathbb{k}$-superalgebras. Finally, $N$-homogeneous superalgebras with $N=2$ are called quadratic superalgebras; for $N=3$, they are called cubic, etc.

3.2. Some examples. In order to explicitly describe a certain $N$-homogeneous superalgebra $\mathcal{A}=A(V, R)$, we will usually fix a $\mathbb{Z}_{2}$-graded $\mathbb{k}$-basis $x_{1}, \ldots, x_{d}$ of $V=\mathcal{A}_{1}$ and denote the parity of $x_{i}$ by $\hat{i}$, as in $\S 1.1$. The $x_{i}$ form a set of algebra generators for $A$. Following Manin [38],[39], the $d$-tuple $\boldsymbol{f}=(\hat{1}, \ldots, \hat{d}) \in \mathbb{Z}_{2}^{d}$ is called the format of the basis $\left\{x_{i}\right\}$.

Example 3.1 (Quantum superspace [39]). For a fixed family $\boldsymbol{q}$ of scalars $0 \neq q_{i j} \in \mathbb{k}$ $(1 \leq i<j \leq d)$ and a given format $\boldsymbol{f}=(\hat{1}, \ldots, \hat{d}) \in \mathbb{Z}_{2}^{d}$ of the basis $x_{1}, \ldots, x_{d}$, the quadratic superalgebra $\mathcal{A}=\mathrm{S}_{\boldsymbol{q}}^{f}$ is defined as the factor of $\mathrm{T}(V)$ modulo the ideal generated by the elements

$$
\begin{gathered}
r_{i}:=x_{i} \otimes x_{i} \in\left(V^{\otimes 2}\right)_{\overline{0}} \quad(\hat{i}=\overline{1}), \\
r_{i j}:=x_{j} \otimes x_{i}-q_{i j}(-1)^{\hat{i} \hat{j}} x_{i} \otimes x_{j} \in\left(V^{\otimes 2}\right)_{\hat{i}+\hat{j}} \quad(i<j) .
\end{gathered}
$$


Thus, the algebra $\mathrm{S}_{\boldsymbol{q}}^{f}$ is generated by $x_{1}, \ldots, x_{d}$ subject to the defining relations

$$
x_{i} x_{i}=0 \quad(\hat{i}=\overline{1})
$$

and

$$
x_{j} x_{i}=q_{i j}(-1)^{\hat{i} \hat{j}} x_{i} x_{j} \quad(i<j) .
$$

In the special case where all $q_{i j}=1$, the algebra $\mathrm{S}_{\boldsymbol{q}}^{\boldsymbol{f}}$ is the symmetric superalgebra $\mathrm{S}(V)$ of $V$ as in Example 2.1.

The ordered monomials of the form $x_{1}^{m_{1}} x_{2}^{m_{2}} \ldots x_{d}^{m_{d}}$, with $\sum_{i} m_{i}=n, m_{i} \geq 0$ for all $i$ and $m_{i} \leq 1$ if $\hat{i}=\overline{1}$, form a $\mathbb{k}$-basis of the $n^{\text {th }}$ homogeneous component of $\mathrm{S}_{\boldsymbol{q}}^{f}$. Therefore,

$$
\operatorname{dim}_{\mathbb{k}}\left(\mathrm{S}_{\boldsymbol{q}}^{\boldsymbol{f}}\right)_{n}=\sum_{r+s=n}\left(\begin{array}{c}
r+p-1 \\
p-1
\end{array}\right)\left(\begin{array}{c}
q \\
s
\end{array}\right)
$$

where $\operatorname{dim} V_{\overline{0}}=p$ and $\operatorname{dim} V_{\overline{1}}=q$ as usual.Thus, the generating series of the dimensions is

$$
\sum_{n \geq 0} \operatorname{dim}_{\mathbb{k}}\left(\mathrm{S}_{\boldsymbol{q}}^{f}\right)_{n} t^{n}=\frac{(1+t)^{q}}{(1-t)^{p}} .
$$

Example 3.2 (Yang-Mills algebras [11], [10]). Fix a collection of elements $x_{1}, \ldots, x_{d}$ $(d \geq 2)$, numbered so as to have parity $\hat{i}=\overline{0}$ for $i \leq p$ and $\hat{i}=\overline{1}$ for $i>p$. Let $G=\left(g_{i j}\right) \in \mathrm{GL}_{d}(\mathbb{k})$ be an invertible symmetric $d \times d$-matrix satisfying $g_{i j}=0$ if $\hat{i} \neq \hat{j}$ and consider the cubic superalgebra $\mathcal{A}$ that is generated by elements $x_{1}, \ldots, x_{d}$ subject to the relations

$$
\sum_{i, j} g_{i j}\left[x_{i},\left[x_{j}, x_{k}\right]\right]=0 \quad(k=1, \ldots, d) .
$$

Here $[\cdot, \cdot]$ is the supercommutator (2.2). The algebra $\mathcal{A}$ will be denoted by $\operatorname{y\mathcal {M}}^{p \mid q}$ $(q=d-p)$. In particular, the pure even algebra $\mathrm{y \mathcal {N }}^{d \mid 0}$ is the ordinary Yang-Mills algebra introduced in [10] while $\mathcal{Y M}^{0 \mid d}$ is the super Yang-Mills algebra as in [11].

As usual, put $V=\sum_{i} \mathbb{k} x_{i}$ and let $[\cdot, \cdot \cdot]_{\otimes}$ denote the supercommutator in $\mathrm{T}(V)$. Furthermore, put $r_{k}=\sum_{i, j} g_{i j}\left[x_{i},\left[x_{j}, x_{k}\right]_{\otimes}\right]_{\otimes}$ and $R=\sum_{k} \mathbb{k} r_{k} \subseteq V^{\otimes 3}$; so $\mathrm{y \mathcal {N }}^{p \mid q}=\mathrm{T}(V) /(R)$. Using the symmetry of $G$, we may replace the $r_{k}$ by simpler relations as follows. Choose an invertible $d \times d$-matrix $C=\left(c_{i j}\right)$ with $c_{i j}=0$ if $\hat{i} \neq \hat{j}$ and such that $C^{\text {tr }} G C$ is diagonal, say $\sum_{i, j} c_{i r} g_{i j} c_{j s}=g_{s} \delta_{s}^{r}$. Replace the bases $\left\{x_{i}\right\}$ of $V$ and $\left\{r_{k}\right\}$ of $R$ by the new bases $y_{i}=\sum_{j} c^{i j} x_{j}$ and $s_{k}=\sum_{k} c^{k \ell} r_{\ell}$ where $C^{-1}=\left(c^{i j}\right)$. Note that $y_{i}$ has parity $\hat{i}$ and $s_{k}$ has parity $\hat{k}$, the parity of $r_{k}$. A simple calculation shows that $s_{k}=\sum_{i \neq k} g_{i}\left[y_{i},\left[y_{i}, y_{k}\right]_{\otimes}\right]_{\otimes}$. Thus we obtain the following defining relations for the generators $y_{1}, \ldots, y_{d}$ of $\mathcal{y \mathcal { M }}^{p \mid q}$ :

$$
\sum_{i \neq k} g_{i}\left[y_{i},\left[y_{i}, y_{k}\right]\right]=0 \quad(k=1, \ldots, d) .
$$


The resulting algebras for $d=2$ are as follows. Putting $x=y_{1}$ and $y=y_{2}$ we have two defining relations: $[x,[x, y]]=0$ and $[y,[y, x]]=0$. In the pure even case $(\hat{x}=\hat{y}=\overline{0})$, the supercommutators are the ordinary Lie commutators. So $y \mathcal{M}^{2 \mid 0}$ is the enveloping algebra of the Heisenberg Lie algebra; see [1], (0.4). In the pure odd case $(\hat{x}=\hat{y}=\overline{1})$, the two relations can be written as $x^{2} y=y x^{2}$ and $y x^{2}=x^{2} y$. The resulting algebra $y \mathcal{X}^{0 \mid 2}$ is a cubic Artin-Schelter algebra of type $S_{1}$ [1], (8.6). Thus, both unmixed algebras are Artin-Schelter regular of global dimension 3. In the mixed case, however $(\hat{x}=\overline{0}, \hat{y}=\overline{1})$, the relations say that $x$ commutes with the Lie commutator $[x, y]$ while $y$ anticommutes: $y[x, y]=-[x, y] y$. Thus, $[x, y]$ is a normal element of $y \mathcal{X}^{1 \mid 1}$ and $\mathcal{Y \mathcal { M }}^{1 \mid 1} /([x, y])$ is a polynomial algebra in two variables over $\mathbb{k}$. Moreover, the calculation

$$
[x, y]^{2}=[x,[x, y] y]=-[x, y[x, y]]=-[x, y]^{2}
$$

shows that $[x, y]^{2}=0$. Thus, the algebra $\mathcal{Y N}^{1 \mid 1}$ is noetherian with Gelfand-Kirillov dimension 2 and infinite global dimension.

Returning to the case of general $d \geq 2$, we now concentrate on the unmixed algebras introduced by Connes and Dubois-Violette. We will denote these algebras

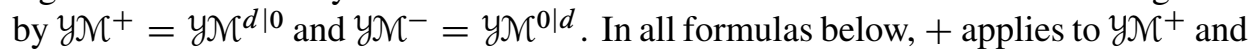
- to $\mathcal{Y M}^{-}$. The generators $s_{k}=\sum_{i \neq k} g_{i}\left[y_{i},\left[y_{i}, y_{k}\right]_{\otimes}\right]_{\otimes}$ of the space of relations $R$ can be written as $s_{k}=\sum_{\ell} y_{\ell} \otimes m_{\ell k}= \pm \sum_{\ell} m_{k \ell} \otimes y_{\ell}$ with

$$
m_{\ell k}= \begin{cases}g_{\ell}\left(y_{\ell} \otimes y_{k}-(1 \pm 1) y_{k} \otimes y_{\ell}\right) & \text { for } \ell \neq k, \\ \pm \sum_{i \neq k} g_{i} y_{i} \otimes y_{i} & \text { for } \ell=k .\end{cases}
$$

Thus, putting $Y=\left(y_{1}, \ldots, y_{d}\right)$ and letting $M$ denote the $d \times d$-matrix over $\mathrm{yMM}^{ \pm}$ whose $(\ell, k)$-entry is the image of $m_{\ell k}$, the defining relations (3.6) can be written as

$$
Y M=0 \quad \text { or } \quad M Y^{\text {tr }}=0 .
$$

The defining relations (3.6) for $\mathcal{A}=\mathrm{y \mathcal {M }}^{-}$amount to the even element $\sum_{i} g_{i} y_{i}^{2} \in \mathcal{A}_{2}$ being central in $\mathcal{A}$.

Example 3.3 ( $N$-symmetric superalgebra; cf. [5]). Let $N \geq 2$ be given and let $V$ be a vector superspace $V$ over a field $\mathbb{k}$ with char $\mathbb{k}=0$ or char $\mathbb{k}>N$. Define

$$
\mathrm{S}_{N}(V)=A(V, R) \quad \text { with } R=\Lambda^{N} V=c_{Y_{N}}\left(V^{\otimes N}\right) \subseteq V^{\otimes N}
$$

where $Y_{N}$ is the antisymmetrizer idempotent of the group algebra $\mathbb{k}\left[\Im_{N}\right]$; see (2.13). This defines a functor $\mathrm{S}_{N}(\cdot)$ : Vect $t_{\mathbb{k}}^{s} \rightarrow \mathrm{H}_{N} \mathrm{Alg}_{\mathbb{k}}^{s}$. Since $2 c_{Y_{2}}$ is the supercommutator in $\mathrm{T}(V)$, the algebra $\mathrm{S}_{2}(V)$ is just the symmetric superalgebra $\mathrm{S}(V)$ of $V$; see Example 3.1. The algebra $\mathrm{S}_{N}(V)$, for a pure even space $V=V_{\overline{0}}$ and general $N \geq 2$, has been introduced in [5]. 
If $2 \leq M \leq N$ then, viewing $\mathbb{k}\left[\subseteq_{M}\right]$ as a subalgebra of $\mathbb{k}\left[\subseteq_{N}\right]$ as usual, the antisymmetrizers of $\mathbb{k}\left[\widetilde{S}_{N}\right]$ and $\mathbb{k}\left[\widetilde{S}_{M}\right]$ satisfy $Y_{N}=Y_{M} a$ for some $a \in \mathbb{k}\left[\widetilde{S}_{N}\right]$. Therefore,

$$
R=c_{Y_{N}}\left(V^{\otimes N}\right) \subseteq c_{Y_{M}}\left(V^{\otimes N}\right)=c_{Y_{M}}\left(V^{\otimes M}\right) \otimes V^{\otimes(N-M)} .
$$

This shows that the identity map on $V$ extends to an epimorphism of superalgebras $\mathrm{S}_{N}(V) \rightarrow \mathrm{S}_{M}(V)$.

Now assume that $\operatorname{dim}_{\mathbb{k}} V=d$ and fix a standard basis $x_{1}, \ldots, x_{d}$ of $V$, with $\hat{i}=\overline{0}$ for $i \leq p$ and $\hat{i}=\overline{1}$ for $i>p$. From the basis for $\Lambda^{N} V$ exhibited in $\S 2.6 .1$ we obtain that the algebra $\mathrm{S}_{N}(V)$ is generated by $x_{1}, \ldots, x_{d}$ subject to the relations

$$
\sum_{\sigma \in \mathbb{S}_{N}}(-1)^{\sum(p, q) \in \operatorname{inv}(\sigma)} \hat{1+\hat{i_{p}} \hat{i_{q}}} x_{i_{\sigma^{-1}(1)}} x_{i_{\sigma^{-1}(2)}} \ldots x_{i_{\sigma^{-1}(N)}}=0
$$

with $1 \leq i_{1}<i_{2}<\cdots<i_{m} \leq p=\operatorname{dim}_{\mathbb{k}} V_{\overline{0}}<i_{m+1} \leq \cdots \leq i_{N} \leq d=\operatorname{dim}_{\mathbb{k}} V$; see formula (2.14).

Example 3.4. The following construction generalizes Example 3.3. Fix $N \geq 2$ and $0 \neq q \in \mathbb{k}$ and assume that condition (1.14) is satisfied. Given a Hecke operator $\mathcal{R}: V^{\otimes 2} \rightarrow V^{\otimes 2}$ on a vector superspace $V$ we define the $N$-homogeneous superalgebra

$$
\wedge_{\mathcal{R}, N}:=A(V, R) \quad \text { with } R=\operatorname{Im} \rho_{\mathcal{R}}\left(X_{N}\right) \subseteq V^{\otimes N}
$$

where $X_{N} \in \mathscr{H}_{N, q}$ is the $q$-symmetrizer (1.16) and $\rho_{\mathcal{R}}$ is the representation (1.20) of $\mathscr{H}_{N, q}$. We also put

$$
\mathrm{S}_{\mathcal{R}, N}:=\Lambda_{-q \mathcal{R}^{-1}, N}=A(V, R) \quad \text { with } R=\operatorname{Im} \rho_{\mathcal{R}}\left(Y_{N}\right) \subseteq V^{\otimes N}
$$

where $Y_{N} \in \mathscr{H}_{N, q}$ is the antisymmetrizer (1.17). The algebra $\mathrm{S}_{N}(V)$ in Example 3.3 is identical with $\mathrm{S}_{c_{V, V}, N}(q=1)$.

3.3. The dual of a homogeneous superalgebra. Let $\mathcal{A}=A(V, R)$ be an $N$-homogeneous superalgebra. The dual $\mathcal{A}^{!}$of $\mathcal{A}$ is defined by

$$
\mathcal{A}^{!}=A\left(V^{*}, R^{\perp}\right)
$$

where $R^{\perp} \subseteq V^{* \otimes N}$ is the (homogeneous) subspace consisting of all elements that vanish on $R \subseteq V^{\otimes N}$, using (1.4) in order to evaluate elements of $V^{* \otimes N}$ on $V^{\otimes N}$. Thus, (3.1) takes the form

$$
\mathcal{A}_{n}^{!}=V^{* \otimes n} / R_{n}^{\perp} \quad \text { with } R_{n}^{\perp}:=\sum_{i+j+N=n} V^{* \otimes i} \otimes R^{\perp} \otimes V^{* \otimes j} .
$$


Identifying $V^{* \otimes n}$ with the linear dual of $V^{\otimes n}$ via (1.4), we have $V^{* \otimes i} \otimes R^{\perp} \otimes V^{* \otimes j}=$ $\left(V^{\otimes j} \otimes R \otimes V^{\otimes i}\right)^{\perp}$. Hence,

$$
R_{n}^{\perp}=\left(\bigcap_{i+j+N=n} V^{\otimes j} \otimes R \otimes V^{\otimes i}\right)^{\perp} .
$$

The canonical isomorphism $V \stackrel{\sim}{\longrightarrow} V^{* *}$ in (1.5) leads to an isomorphism $V^{\otimes N} \stackrel{\sim}{\longrightarrow} V^{* * \otimes N}$ which maps $R$ onto $R^{\perp \perp}$. Hence,

$$
\mathcal{A}^{! !} \cong \mathcal{A} \text {. }
$$

Moreover, if $f: \mathcal{A}=A(V, R) \rightarrow \mathcal{A}^{\prime}=A\left(V^{\prime}, R^{\prime}\right)$ is any morphism in $\mathrm{H}_{N} \mathrm{Alg}_{\mathbb{k}}^{s}$ then the transpose of $f_{1}: V \rightarrow V^{\prime}$ induces a morphism $f^{!}:\left(\mathcal{A}^{\prime}\right)^{!} \rightarrow \mathcal{A}^{!}$in $\mathrm{H}_{N} \mathrm{Alg}_{\mathbb{k}}^{s}$. Thus, we have a contravariant quasi-involutive dualization functor $\mathcal{A} \mapsto \mathcal{A}^{!}, f \mapsto f^{!}$on $\mathrm{H}_{N} \mathrm{Alg}_{\mathfrak{k}}^{s}$.

Example 3.5. The dual of $A(V, 0)=\mathrm{T}(V)$ is $A\left(V^{*}, V^{* \otimes N}\right)$; so

$$
\mathrm{T}(V)^{!}=\mathrm{T}\left(V^{*}\right) /\left(V^{* \otimes N}\right)
$$

In particular, letting $V=\mathbb{k}$ be the unit object of Vect $t_{\mathbb{k}}^{s}$, we have $A(\mathbb{k}, 0)=\mathbb{k}[t]$ (polynomial algebra) and $A(\mathbb{k}, 0)^{!}=\mathbb{k}[d] /\left(d^{N}\right)$, with $t$ and $d$ both having degree 1 and parity $\overline{0}$.

Example 3.6 (Dual of quantum superspace). We will describe the dual $\mathcal{A}^{!}$of quantum superspace $\mathcal{A}=\mathrm{S}_{\boldsymbol{q}}^{f}$; see Example 3.1. Fix a homogeneous $\mathbb{k}$-basis $x_{1}, \ldots, x_{d}$ with format $f$ for $V$, and let $x^{1}, \ldots, x^{d}$ denote the dual basis of $V^{*}$; this basis also has format $\boldsymbol{f}$. Evaluating an arbitrary element $f=\sum_{\ell, m} f_{\ell m} x^{\ell} \otimes x^{m} \in V^{* \otimes 2}$ on one of the generating relations $r_{i}, r_{i j} \in R$ in (3.2), (3.3) we obtain $\left\langle f, r_{i}\right\rangle=f_{i i}$ and $\left\langle f, r_{i j}\right\rangle=f_{i j}-q_{i j}(-1)^{\hat{i} \hat{j}} f_{j i}$. Therefore, the space $R^{\perp} \subseteq V^{* \otimes 2}$ has a basis consisting of the elements $s^{\ell}:=x^{\ell} \otimes x^{\ell}(\hat{\ell}=\overline{0})$ and $s^{\ell, k}:=x^{\ell} \otimes x^{k}+q_{k \ell}(-1)^{\hat{k} \hat{\ell}} x^{k} \otimes x^{\ell}$ $(k<\ell)$. In summary, $\mathcal{A}^{!}$is generated by $x^{1}, \ldots, x^{d}$ subject to the defining relations

$$
x^{\ell} x^{\ell}=0 \quad(\hat{\ell}=\overline{0})
$$

and

$$
x^{\ell} x^{k}=-q_{k \ell}(-1)^{\hat{k} \hat{\ell}} x^{k} x^{\ell} \quad(k<\ell) .
$$

Thus, $\mathcal{A}^{!}$is isomorphic to quantum superspace $\mathrm{S}_{\boldsymbol{q}^{\prime}}^{\boldsymbol{f}^{\prime}}$ with $q_{i j}^{\prime}=(-1)^{\hat{i}+\hat{j}} q_{i j}$ and $\boldsymbol{f}^{\prime}=\boldsymbol{f}+(\overline{1}, \ldots, \overline{1})$ the format obtained from $\boldsymbol{f}$ by parity reversal in all components.

Example 3.7 (Duals of the Yang-Mills algebras). Continuing with the notation of Example 3.2, we now describe the algebra $\mathcal{A}^{!}$for $\mathcal{A}=$ y $\mathcal{N}^{p \mid q}$. We assume that char $\mathbb{k}=0$ and work with generators $y_{1}, \ldots, y_{d}$ of $\mathcal{A}$ satisfying (3.6). 
Let $y^{1}, \ldots, y^{d}$ denote the basis of $V^{*}$ given by $\left\langle y^{i}, y_{j}\right\rangle=\delta_{j}^{i}$ and put $\gamma=$ $\frac{1}{d-1} \sum_{i} g_{i}^{-1} y^{i} \otimes y^{i} \in V^{* \otimes 2}$. Then, for the generators $s_{k}=\sum_{i \neq k} g_{i}\left[y_{i},\left[y_{i}, y_{k}\right]_{\otimes}\right]_{\otimes}$ of $R$ as in Example 3.2, one computes

$$
\begin{aligned}
\left\langle y^{a} \otimes y^{b} \otimes y^{c}, s_{k}\right\rangle & =g_{c} \delta_{b}^{c} \delta_{k}^{a}+(-1)^{\hat{b}} g_{b} \delta_{a}^{b} \delta_{k}^{c}-(-1)^{\hat{a} \hat{k}}\left(1+(-1)^{\hat{a}}\right) g_{a} \delta_{c}^{a} \delta_{k}^{b}, \\
\left\langle y^{i} \otimes \gamma, s_{k}\right\rangle & =\delta_{k}^{i} .
\end{aligned}
$$

Therefore, the map $\varphi \mapsto \varphi-\sum_{k}\left\langle\varphi, s_{k}\right\rangle y^{k} \otimes \gamma$ is an epimorphism $V^{* \otimes 3} \rightarrow R^{\perp} \subset$ $V^{* \otimes 3}$. We obtain that the algebra $\mathcal{A}^{!}$is generated by $y^{1}, \ldots, y^{d}$ subject to the relations

$$
y^{a} y^{b} y^{c}=\left(g_{c} \delta_{b}^{c} y^{a}+(-1)^{\hat{b}} g_{b} \delta_{a}^{b} y^{c}-(-1)^{\hat{a} \hat{b}}\left(1+(-1)^{\hat{a}}\right) g_{a} \delta_{c}^{a} y^{b}\right) \boldsymbol{g}
$$

where $\boldsymbol{g}=\frac{1}{d-1} \sum_{i} g_{i}^{-1} y^{i} y^{i}$ is the image of $\gamma$ in $\mathcal{A}$.

Since $\mathcal{A}^{!}$is 3-homogeneous, we clearly have $\mathcal{A}_{0}^{!}=\mathbb{k}, \mathcal{A}_{1}^{!}=\bigoplus_{i} \mathbb{k} y^{i}=V^{*}$ and $\mathcal{A}_{2}^{!}=\bigoplus_{i, j} \mathbb{k} y^{i} y^{j} \cong V^{* \otimes 2}$. By (3.13), the elements $y^{a} \boldsymbol{g}$ form a $\mathbb{k}$-basis of $\mathcal{A}_{3}^{!}=V^{* \otimes 3} / R^{\perp} \cong R^{*}$. Using the defining relations (3.14) it is not hard to see that $\mathcal{A}_{4}^{!}=\mathbb{k} \boldsymbol{g}^{2}$ and $\mathcal{A}_{n}^{!}=0$ for $n \geq 5$. If $\mathcal{A}=\mathrm{y \mathcal {M }}^{p \mid q}$ is of mixed type (i.e., $p \neq 0$ and $q \neq 0$ ) then $\boldsymbol{g}^{2}=0$.

Example 3.8 (Dual of the $N$-symmetric superalgebra). Recall from Example 3.3 that $\mathrm{S}_{N}(V)=A(V, R)$ with $R=c_{Y_{N}}\left(V^{\otimes N}\right)$. Since $Y_{N}$ is central in $\mathbb{k}\left[\subseteq_{N}\right]$ and stable under the inversion involution ${ }^{*}$ of $\mathbb{k}\left[\subseteq_{N}\right]$, it follows from (1.11) that

$$
\left\langle x, c_{Y_{N}}(y)\right\rangle=\left\langle c_{Y_{N}}(x), y\right\rangle
$$

holds for all $x \in V^{* \otimes N}$ and $y \in V^{\otimes N}$. Therefore,

$$
R^{\perp}=\operatorname{Ker}_{V^{* \otimes N}}\left(c_{Y_{N}}\right)=\left(1-c_{Y_{N}}\right)\left(V^{* \otimes N}\right)
$$

and so

$$
\mathrm{S}_{N}(V)^{!}=A\left(V^{*},\left(1-c_{Y_{N}}\right)\left(V^{* \otimes N}\right)\right) .
$$

Note that

$$
\bigcap_{i+j+N=n} V^{\otimes i} \otimes R \otimes V^{\otimes j}=c_{Y_{n}}\left(V^{\otimes n}\right)
$$

holds for all $n \geq N$. This follows from (1.19). Alternatively, as has been noted in Example 3.3, we have $c_{Y_{n}}\left(V^{\otimes n}\right) \subseteq R \otimes V^{\otimes(n-N)}$. In the same way, one sees that $c_{Y_{n}}\left(V^{\otimes n}\right) \subseteq V^{\otimes i} \otimes R \otimes V^{\otimes j}$ whenever $i+j+N=n$. For the reverse inclusion, note that each $x \in V^{\otimes i} \otimes R \otimes V^{\otimes j}$ satisfies $c_{\sigma_{\ell}}(x)=-x$ for all transpositions $\sigma_{\ell}=(\ell, \ell+1) \in \Im_{n}$ with $i<\ell<i+N$. Hence, the left-hand side of (3.15) 
is contained in the space of antisymmetric $n$-tensors, $\Lambda^{n} V=c_{Y_{n}}\left(V^{\otimes n}\right)$, thereby proving (3.15). We deduce from (3.10), (3.11) and (2.15) that

$$
\operatorname{dim}_{\mathbb{k}} \mathrm{S}_{N}(V)_{n}^{!}= \begin{cases}d^{n} & \text { if } n<N, \\
\sum_{r+s=n}\left(\begin{array}{c}
p \\
r
\end{array}\right)\left(\begin{array}{c}
q+s-1 \\
s
\end{array}\right) & \text { if } n \geq N,\end{cases}
$$

where $d=\operatorname{dim}_{\mathbb{k}} V, p=\operatorname{dim}_{\mathbb{k}} V_{\overline{0}}$ and $q=\operatorname{dim}_{\mathbb{k}} V_{\overline{1}}$.

3.4. The operations $\circ$ and $\bullet$ on $\mathbf{H}_{N} \mathbf{A} \mathbf{g l}_{\mathbb{k}}^{s}$. Let $\mathcal{A}=A(V, R)$ and $\mathcal{A}^{\prime}=A\left(V^{\prime}, R^{\prime}\right)$ be $N$-homogeneous superalgebras. Following [37] and [7] we define the white and black products $\mathcal{A} \circ \mathcal{A}^{\prime}$ and $\mathcal{A} \bullet \mathcal{A}^{\prime}$ by

$$
\begin{aligned}
& \mathcal{A} \circ \mathcal{A}^{\prime}=A\left(V \otimes V^{\prime}, c_{\pi_{N}}\left(R \otimes V^{\prime \otimes N}+V^{\otimes N} \otimes R^{\prime}\right)\right), \\
& \mathcal{A} \bullet \mathcal{A}^{\prime}=A\left(V \otimes V^{\prime}, c_{\pi_{N}}\left(R \otimes R^{\prime}\right)\right)
\end{aligned}
$$

where $\pi_{N} \in \widetilde{S}_{2 N}$ is the inverse of the permutation

$$
(1,2, \ldots, 2 N) \mapsto(1, N+1,2, N+2, \ldots, k, N+k, \ldots, N, 2 N) .
$$

Explicitly, $c_{\pi_{N}}: V^{\otimes N} \otimes V^{\prime \otimes N} \rightarrow\left(V \otimes V^{\prime}\right)^{\otimes N}$ is the morphism in $\operatorname{Vect}_{\mathbb{k}}^{s}$ that is given by

$c_{\pi_{N}}\left(v_{1} \otimes \ldots v_{N} \otimes v_{1}^{\prime} \otimes \cdots \otimes v_{N}^{\prime}\right)=(-1)^{\sum_{i} \sum_{j>i} \hat{v}_{i}^{\prime} \hat{v}_{j}}\left(v_{1} \otimes v_{1}^{\prime}\right) \otimes \cdots \otimes\left(v_{N} \otimes v_{N}^{\prime}\right)$

Hence, $c_{\pi_{N}}\left(R \otimes R^{\prime}\right)$ and $c_{\pi_{N}}\left(R \otimes V^{\prime \otimes N}+V^{\otimes N} \otimes R^{\prime}\right)$ are homogeneous subspaces of $\left(V \otimes V^{\prime}\right)^{\otimes N}$ and so $\mathcal{A} \circ \mathcal{A}^{\prime}$ and $\mathcal{A} \bullet \mathcal{A}^{\prime}$ belong to $\mathrm{H}_{N} \mathrm{Alg}_{\mathbb{k}}^{s}$.

Under the isomorphism $\left(V^{*} \otimes V^{*}\right)^{\otimes N} \stackrel{\sim}{\longrightarrow}\left(V \otimes V^{\prime}\right)^{* \otimes N}$ which comes from (1.4), the relations $c_{\pi_{N}}\left(R^{\prime \perp} \otimes R^{\perp}\right)$ of $\mathcal{A}^{\prime !} \bullet \mathcal{A}^{!}$map onto the relations $\left(c_{\pi_{N}}\left(R \otimes V^{\prime \otimes N}+V^{\otimes N} \otimes R^{\prime}\right)\right)^{\perp}$ of $\left(\mathcal{A} \circ \mathcal{A}^{\prime}\right) !$. In fact, by (1.11) we have $c_{\pi_{N}}^{*}=c_{\pi_{N}}$ because $\pi_{N} \tau=\tau \pi_{N}$, and consequently $\langle x, y\rangle=\left\langle c_{\pi_{N}}(x), c_{\pi_{N}}(y)\right\rangle$ holds for all $x \in V^{* \otimes N} \otimes V^{* \otimes N}$ and $y \in V^{\otimes N} \otimes V^{\prime \otimes N}$. Therefore, canonically,

$$
\left(\mathcal{A} \circ \mathcal{A}^{\prime}\right)^{!} \cong \mathcal{A}^{\prime !} \bullet \mathcal{A}^{!} \quad \text { and } \quad\left(\mathcal{A} \bullet \mathcal{A}^{\prime}\right)^{!} \cong \mathcal{A}^{\prime !} \circ \mathcal{A}^{!},
$$

the two identities being equivalent by (3.12).

By definition of $\circ$, the canonical isomorphisms $\mathbb{k} \otimes V \cong V \cong V \otimes \mathbb{k}$ in $\operatorname{Vect}_{\mathbb{k}}^{s}$ give isomorphisms $A(\mathbb{k}, 0) \circ \mathcal{A} \cong \mathcal{A} \cong \mathcal{A} \circ A(\mathbb{k}, 0)$ in $\mathrm{H}_{N} \mathrm{Alg}_{\mathbb{k}}^{s}$, and (3.18) yields similar isomorphisms for $\bullet$, with $A(\mathbb{k}, 0)^{!}=\mathbb{k}[d] /\left(d^{N}\right)$ replacing $A(\mathbb{k}, 0)=\mathbb{k}[t]$; see Example 3.5.

The supersymmetry isomorphism $c_{V, V^{\prime}}: V \otimes V^{\prime} \stackrel{\sim}{\longrightarrow} V^{\prime} \otimes V$ in $\operatorname{Vect}_{\mathbb{k}}^{s}($ see (1.1)) yields isomorphisms

$$
\mathcal{A} \circ \mathcal{A}^{\prime} \cong \mathcal{A}^{\prime} \circ \mathcal{A} \quad \text { and } \quad \mathcal{A} \bullet \mathcal{A}^{\prime} \cong \mathcal{A}^{\prime} \bullet \mathcal{A}
$$


in $\mathrm{H}_{N} \mathrm{Alg}_{\mathbb{k}}^{s}$. To see this, note that the following diagram of isomorphisms in $\operatorname{Vect}_{\mathbb{k}}^{S}$ commutes:

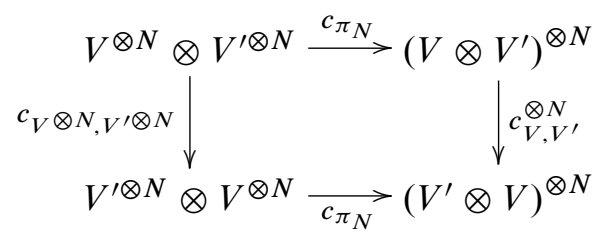

with $v_{1} \otimes \ldots v_{N} \otimes v_{1}^{\prime} \otimes \ldots v_{N}^{\prime} \mapsto(-1)^{\sum_{i} \sum_{j \geq i} \hat{v}_{i}^{\prime} \hat{v}_{j}}\left(v_{1}^{\prime} \otimes v_{1}\right) \otimes \ldots\left(v_{N}^{\prime} \otimes v_{N}\right)$ in

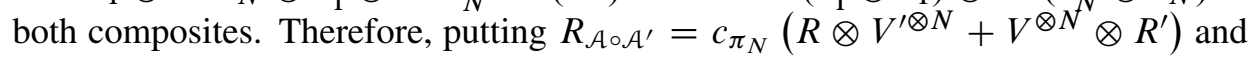
similarly for $R_{\mathcal{A}^{\prime} \circ \mathcal{A}}$ etc., we have

$$
\begin{aligned}
c_{V, V^{\prime}}^{\otimes N}\left(R_{\mathcal{A} \circ \mathcal{A}^{\prime}}\right) & =\left(c_{\pi_{N}} \circ c_{V^{\otimes N}, V^{\prime} \otimes N}\right)\left(R \otimes V^{\prime \otimes N}+V^{\otimes N} \otimes R^{\prime}\right) \\
& =c_{\pi_{N}}\left(R^{\prime} \otimes V^{\otimes N}+V^{\prime \otimes N} \otimes R\right) \\
& =R_{\mathcal{A}^{\prime} \circ \mathcal{A}} .
\end{aligned}
$$

Similarly, one sees that $c_{V, V^{\prime}}^{\otimes N}\left(R_{\mathcal{A} \bullet \mathcal{A}^{\prime}}\right)=R_{\mathcal{\mathcal { H } ^ { \prime }} \bullet \mathcal{A}}$. This proves (3.19).

In the same way, the associativity isomorphism $a_{V, V^{\prime}, V^{\prime \prime}}:\left(V \otimes V^{\prime}\right) \otimes V^{\prime \prime} \cong$ $V \otimes\left(V^{\prime} \otimes V^{\prime \prime}\right)$ in $\operatorname{Vect}_{\mathbb{k}}^{S}$ leads to isomorphisms

$$
\left(\mathcal{A} \circ \mathcal{A}^{\prime}\right) \circ \mathcal{A}^{\prime \prime} \cong \mathcal{A} \circ\left(\mathcal{A}^{\prime} \circ \mathcal{A}^{\prime \prime}\right) \quad \text { and } \quad\left(\mathcal{A} \bullet \mathcal{A}^{\prime}\right) \bullet \mathcal{A}^{\prime \prime} \cong \mathcal{A} \bullet\left(\mathcal{A}^{\prime} \bullet \mathcal{A}^{\prime \prime}\right)
$$

in $\mathrm{H}_{N} \mathrm{Alg}_{\mathbb{k}}^{s}$. This is a consequence of the following commutative diagram of isomorphisms in $\operatorname{Vect}_{\mathbb{k}}^{s}$ :

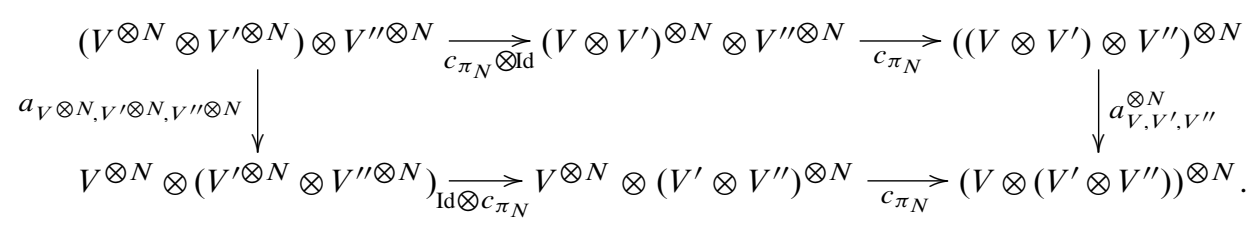

Finally, the compatibility between the isomorphisms $c_{V, V^{\prime}}$ and $a_{V, V^{\prime}, V^{\prime \prime}}$ (see $\S 1.2$ ) is inherited by the isomorphisms (3.19) and (3.20) in $\mathrm{H}_{N} \mathrm{Alg}_{\mathfrak{k}}^{s}$. To summarize:

Proposition 3.9. The operations $\circ$ and $\bullet$ both make the category $\mathrm{H}_{N} \mathrm{Alg}_{\mathbb{k}}^{S}$ of $N$ homogeneous $\mathbb{k}$-superalgebras into a symmetric tensor category, with unit objects $A(\mathbb{k}, 0)=\mathbb{k}[t]$ for $\circ$ and $A(\mathbb{k}, 0)^{!}=\mathbb{k}[d] /\left(d^{N}\right)$ for $\bullet$.

3.5. The superalgebra map $i: \mathcal{A} \circ \mathcal{A}^{\prime} \rightarrow \mathcal{A} \otimes \mathcal{A}^{\prime}$. Let $\mathcal{A}=A(V, R)$ and $\mathcal{A}^{\prime}=$ $A\left(V^{\prime}, R^{\prime}\right)$ be objects of $\mathrm{H}_{N} A_{g_{\mathbb{k}}}^{s}$. The superalgebra $\mathcal{A} \otimes \mathcal{A}^{\prime}$ is generated by $V \oplus V^{\prime}$ subject to the relations

$$
R+R^{\prime} \subseteq\left(V \oplus V^{\prime}\right)^{\otimes N} \quad \text { and } \quad\left[V, V^{\prime}\right]_{\otimes} \subseteq\left(V \oplus V^{\prime}\right)^{\otimes 2}
$$


where $[\cdot, \cdot]_{\otimes}$ is the supercommutator (2.2) in the tensor algebra, as usual. Thus, $\mathcal{A} \otimes \mathcal{A}^{\prime}$ is not $N$-homogeneous when $N \geq 3$. Nonetheless, there always is an injective superalgebra homomorphism $i: \mathcal{A} \circ \mathcal{A}^{\prime} \rightarrow \mathcal{A} \otimes \mathcal{A}^{\prime}$ which is defined as follows. The linear embedding $V \otimes V^{\prime} \hookrightarrow \mathrm{T}(V) \otimes \mathrm{T}\left(V^{\prime}\right)$ extends uniquely to a superalgebra map

$$
\tilde{\imath}: \mathrm{T}\left(V \otimes V^{\prime}\right) \rightarrow \mathrm{T}(V) \otimes \mathrm{T}\left(V^{\prime}\right)
$$

which doubles degrees: the restriction of $\tilde{\imath}$ to degree $n$ is the embedding

$$
\mathrm{T}\left(V \otimes V^{\prime}\right)_{n}=\left(V \otimes V^{\prime}\right)^{\otimes n} \stackrel{c_{\pi_{n}}^{-1}}{\longrightarrow} V^{\otimes n} \otimes V^{\prime \otimes n} \subseteq\left(\mathrm{T}(V) \otimes \mathrm{T}\left(V^{\prime}\right)\right)_{2 n}
$$

in Vect $\mathbb{k}_{\mathbb{k}}^{S}$, where $c_{\pi_{n}}$ is as in (3.17). Thus, $\tilde{\iota}$ identifies the superalgebra $\mathrm{T}\left(V \otimes V^{\prime}\right)$ with the (super) Segre product $\bigoplus_{n \geq 0} V^{\otimes n} \otimes V^{\prime \otimes n}$ of $\mathrm{T}(V)$ and $\mathrm{T}\left(V^{\prime}\right)$.

The map $\tilde{\iota}$ sends $R_{\mathcal{A} \circ \mathcal{A}^{\prime}}=c_{\pi_{N}}\left(R \otimes V^{\prime \otimes N}+V^{\otimes N} \otimes R^{\prime}\right) \subseteq\left(V \otimes V^{\prime}\right)^{\otimes N}$ to $R \otimes V^{\prime \otimes N}+V^{\otimes N} \otimes R^{\prime}$, the kernel of the canonical epimorphism $V^{\otimes N} \otimes V^{\prime \otimes N} \rightarrow$ $\mathcal{A}_{N} \otimes \mathcal{A}_{N}^{\prime}$. Thus:

Proposition 3.10. The algebra map $\tilde{\imath}$ in (3.21) passes down to yield an injective homomorphism $\mathbb{k}$-superalgebras $i: \mathcal{A} \circ \mathcal{A}^{\prime} \longrightarrow \mathcal{A} \otimes \mathcal{A}^{\prime}$ which doubles degree. The image of $i$ is the super Segre product $\bigoplus_{n \geq 0} \mathcal{A}_{n} \otimes \mathcal{A}_{n}^{\prime}$ of $\mathcal{A}$ and $\mathcal{A}^{\prime}$.

3.6. Internal Hom. The isomorphisms (1.3) and (1.4) together with associativity lead to a functorial isomorphism

$$
\operatorname{Hom}_{\mathbb{k}}\left(U \otimes V, W^{*}\right) \cong \operatorname{Hom}_{\mathbb{k}}\left(U,(V \otimes W)^{*}\right)
$$

in $\operatorname{Vect}_{\mathbb{k}}^{s}$. Explicitly, if $g \in \operatorname{Hom}_{\mathbb{k}}\left(U \otimes V, W^{*}\right)$ and $g^{\prime} \in \operatorname{Hom}_{\mathbb{k}}\left(U,(V \otimes W)^{*}\right)$ correspond to each other under the above isomorphism then

$$
\langle g(u \otimes v), w\rangle=\left\langle g^{\prime}(u), v \otimes w\right\rangle
$$

holds for all $u \in U, v \in V$ and $w \in W$.

In particular, by restricting to $\overline{0}$-components, we have a $\mathbb{k}$-linear isomorphism

$$
\operatorname{Hom}_{\text {Vect }}\left(U \otimes V, W^{*}\right) \cong \operatorname{Hom}_{\text {Vect }}\left(U,(V \otimes W)^{*}\right) .
$$

This isomorphism leads to

Proposition 3.11. There is a functorial isomorphism

$$
\operatorname{Hom}_{\mathrm{H}_{N} \operatorname{Alg}_{\mathbb{k}}^{s}}(\mathcal{A} \bullet \mathcal{B}, \mathcal{C}) \cong \operatorname{Hom}_{\mathrm{H}_{N}} \operatorname{Alg}_{\mathbb{k}}^{s}\left(\mathcal{A}, \mathcal{C} \circ \mathcal{B}^{!}\right)
$$


Proof. We follow Manin [37], 4.2. Let $\mathcal{A}=A(U, R), \mathcal{B}=A(V, S)$ and $\mathcal{C}=$ $A(W, T)$ be $N$-homogeneous superalgebras. We will prove the proposition in the following equivalent form; see (3.12) and (3.18):

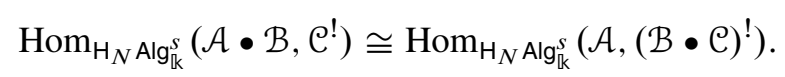

Recall that $\mathcal{C}^{!}=A\left(W^{*}, T^{\perp}\right)$ and $(\mathcal{B} \bullet \mathcal{C})^{!}=A\left((V \otimes W)^{*},\left(c_{\pi_{N}}(S \otimes T)\right)^{\perp}\right)$. Let $g: U \otimes V \rightarrow W^{*}$ be a morphism in Vect $t_{\mathrm{k}}^{s}$ and let $g^{\prime}: U \rightarrow(V \otimes W)^{*}$ be the morphism in $V t_{k \mathrm{k}}^{S}$ that corresponds to $g$ under (3.23). We must show that, for homogeneous subspaces $R \subseteq U^{\otimes N}, S \subseteq V^{\otimes N}$ and $T \subseteq W^{\otimes N}$,

$$
g^{\otimes N}\left(c_{\pi_{N}}(R \otimes S)\right) \subseteq T^{\perp} \Leftrightarrow g^{\prime \otimes N}(R)\left(c_{\pi_{N}}(S \otimes T)\right)^{\perp} .
$$

Identifying $T^{\perp \perp}$ with $T$ as in $\S 3.3$, the first inclusion is equivalent to

$$
\left\langle g^{\otimes N}\left(c_{\pi_{N}}(R \otimes S)\right), T\right\rangle=0,
$$

while the second inclusion states that

$$
\left\langle g^{\prime \otimes N}(R), c_{\pi_{N}}(S \otimes T)\right\rangle=0 .
$$

But (3.22) shows that (3.24) and (3.25) are equivalent, which proves the proposition.

Proposition 3.11 says that the tensor category $\left(\mathrm{H}_{N} \mathrm{Alg}_{\mathbb{k}}^{s}, \bullet\right)$ has an internal $\underline{\mathrm{Hom}}$ which is given by

$$
\underline{\operatorname{Hom}}(\mathcal{A}, \mathcal{B})=\mathcal{B} \circ \mathcal{A}^{!} .
$$

Explicitly, $\operatorname{Hom}(\mathcal{A}, \mathcal{B})$ is an object of $\mathrm{H}_{N} \mathrm{Alg}_{\mathbb{k}}^{s}$ which represents the functor $\left(\mathrm{H}_{N} \mathrm{Alg}_{\mathbb{k}}^{s}\right)^{\text {op }} \rightarrow$ Sets, $X \mapsto \operatorname{Hom}_{\mathrm{H}_{N} \mathrm{Alg}_{\mathrm{k}}^{s}}(X \bullet \mathcal{A}, \mathcal{B})$; so there is an isomorphism of functors

$$
\operatorname{Hom}_{\mathrm{H}_{N} \mathrm{Alg}_{\mathrm{k}}^{s}}(? \bullet \mathcal{A}, \mathcal{B}) \cong \operatorname{Hom}_{\mathrm{H}_{N} \operatorname{Alg}_{\mathrm{k}}^{s}}(?, \underline{\operatorname{Hom}}(\mathcal{A}, \mathcal{B})) .
$$

By general properties of $\underline{\operatorname{Hom}}$ (see [12], Def. 1.6), the morphism $\operatorname{Id}_{\underline{H o m}(\mathcal{A}, \mathcal{B})}$ corresponds to a morphism

$$
\mu: \underline{\operatorname{Hom}}(\mathcal{A}, \mathcal{B}) \bullet \mathcal{A} \rightarrow \mathcal{B}
$$

in $\mathrm{H}_{N} \mathrm{Alg}_{\mathfrak{k}}^{S}$ satisfying the following universal property: for any morphism $f: \mathcal{X} \bullet \mathcal{A} \rightarrow \mathcal{B}$ in $\mathrm{H}_{N} \operatorname{Alg}_{\mathbb{k}}^{S}$ there exists a unique morphism $g: X \rightarrow \underline{\operatorname{Hom}}(\mathcal{A}, \mathcal{B})$ such that the following diagram commutes:

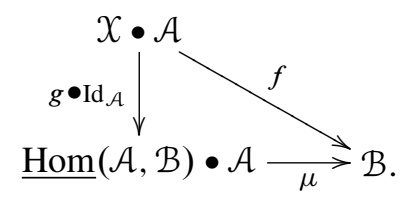


In degree 1, the map $\mu$ is simply $\operatorname{Id}_{V} \otimes \mathrm{ev}_{U}: V \otimes U^{*} \otimes U \rightarrow V \otimes \mathbb{k}=V$.

$\operatorname{From} \underline{\operatorname{Hom}}(\mathcal{B}, \mathcal{C}) \bullet \underline{\operatorname{Hom}}(\mathcal{A}, \mathcal{B}) \bullet \mathcal{A} \stackrel{\operatorname{Id} \bullet \mu}{\longrightarrow} \underline{\operatorname{Hom}}(\mathcal{B}, \mathcal{C}) \bullet \mathcal{B} \stackrel{\mu}{\rightarrow} \mathcal{C}$ one obtains in this way a composition morphism

$$
m: \underline{\operatorname{Hom}}(\mathcal{B}, \mathcal{C}) \bullet \underline{\operatorname{Hom}}(\mathcal{A}, \mathcal{B}) \rightarrow \underline{\operatorname{Hom}}(\mathcal{A}, \mathcal{C})
$$

in $\mathrm{H}_{N} \mathrm{Alg}_{\mathbb{k}}^{s}$. The morphisms $\mu$ and $m$ satisfy the obvious associativity properties.

3.7. The superbialgebra end $\mathcal{A}$. Following Manin [37], 4.2, we define

$$
\underline{\operatorname{hom}}(\mathcal{A}, \mathcal{B})=\underline{\operatorname{Hom}}\left(\mathcal{A}^{!}, \mathcal{B}^{!}\right)^{!}=\mathcal{A}^{!} \cdot \mathcal{B}
$$

for $\mathcal{A}, \mathcal{B}$ in $\mathrm{H}_{N} \mathrm{Alg}_{\mathbb{k}}^{S}$. Applying the dualization functor to (3.26), (3.27) and recalling (3.18), we obtain morphisms

$$
\begin{aligned}
\delta_{\circ}: \mathcal{A} \rightarrow \mathcal{B} \circ \underline{\operatorname{hom}}(\mathcal{B}, \mathcal{A}), \\
\Delta_{\circ}: \underline{\operatorname{hom}(\mathcal{A}, \mathcal{C}) \rightarrow \underline{\operatorname{hom}}(\mathcal{A}, \mathcal{B}) \circ \underline{\operatorname{hom}}(\mathcal{B}, \mathcal{C})}
\end{aligned}
$$

in $\mathrm{H}_{N} \mathrm{Alg}_{\mathfrak{k}}^{s}$. The associativity properties of $\mu$ and $m$ translate into corresponding coassociativity properties for $\delta_{\circ}$ and $\Delta_{\circ}$. Following $\delta_{\circ}$ and $\Delta_{\circ}$ by the algebra map $i$ of Proposition 3.10, we obtain superalgebra maps

$$
\begin{aligned}
\delta: \mathcal{A} \rightarrow \mathcal{B} \otimes \underline{\operatorname{hom}}(\mathcal{B}, \mathcal{A}), \\
\Delta: \underline{\operatorname{hom}(\mathcal{A}, \mathcal{C}) \rightarrow \underline{\operatorname{hom}}(\mathcal{A}, \mathcal{B}) \otimes \underline{\operatorname{hom}}(\mathcal{B}, \mathcal{C}) .}
\end{aligned}
$$

Now take $\mathcal{A}=\mathcal{B}=\mathcal{C}=A(V, R)$ and put end $\mathcal{A}=\underline{\operatorname{hom}}(\mathcal{A}, \mathcal{A})$; so

$$
\underline{\text { end }} \mathcal{A}=\mathcal{A}^{!} \bullet \mathcal{A}=A\left(V^{*} \otimes V, c_{\pi_{N}}\left(R^{\perp} \otimes R\right)\right) .
$$

Then (3.29) yields a coassociative superalgebra map

$$
\Delta: \underline{\text { end }} \mathcal{A} \rightarrow \underline{\text { end }} \mathcal{A} \otimes \underline{\text { end }} \mathcal{A} .
$$

Moreover, by Proposition 3.11, the morphism $\mathcal{A}^{!} \stackrel{\text { Id }}{\rightarrow} \mathcal{A}^{!} \cong \mathbb{k}[t] \circ \mathcal{A}^{!}$corresponds to a morphism end $\mathcal{A}=\mathcal{A}^{!} \bullet \mathcal{A} \rightarrow \mathbb{k}[t]$ in $\mathrm{H}_{N} \mathrm{Alg}_{\mathbb{k}}^{s}$. Following this morphism by the map $t \mapsto 1$ we obtain a superalgebra map

$$
\varepsilon: \underline{\text { end }} \mathcal{A} \rightarrow \mathbb{k}
$$

which in degree 1 is the usual evaluation pairing $\mathrm{ev}_{V}: V^{*} \otimes V \rightarrow \mathbb{k}$ in $\operatorname{Vect}_{\mathbb{k}}^{S}$. Finally, (3.28) provides us with a superalgebra map

$$
\delta_{\mathcal{A}}: \mathcal{A} \rightarrow \mathcal{A} \otimes \underline{\text { end }} \mathcal{A} .
$$


Note that $\delta_{\mathcal{A}}$ maps the degree $n$-component of $\mathcal{A}$ according to

$$
\mathcal{A}_{n} \stackrel{\delta_{\circ}}{\rightarrow}(\mathcal{A} \circ \underline{\text { end }} \mathcal{A})_{n} \stackrel{i}{\rightarrow} \mathcal{A}_{n} \otimes(\underline{\text { end }} \mathcal{A})_{n} \hookrightarrow \mathcal{A}_{n} \otimes \underline{\text { end }} \mathcal{A}
$$

Fixing a graded $k$-basis $x_{1}, \ldots, x_{d}$ of $V$ and denoting the dual basis of $V^{*}$ by $x^{1}, \ldots, x^{d}$ as before, end $\mathcal{A}$ has algebra generators

$$
z_{j}^{i}:=x^{i} \otimes x_{j}
$$

of degree 1 and parity $\hat{i}+\hat{j}$. In terms of these generators, the maps $\varepsilon, \delta_{\mathcal{A}}$ and $\Delta$ are given by

$$
\begin{array}{ll}
\varepsilon\left(z_{i}^{j}\right)=\delta_{i}^{j} & \text { or } \quad \varepsilon(Z)=1, \\
\delta_{\mathcal{A}}\left(x_{j}\right)=\sum_{i} x_{i} \otimes z_{j}^{i} & \text { or } \quad \delta_{\mathcal{A}}\left(x_{1}, \ldots, x_{d}\right)=\left(x_{1}, \ldots, x_{d}\right) \otimes Z, \\
\Delta\left(z_{j}^{i}\right)=\sum_{k} z_{k}^{i} \otimes z_{j}^{k} & \text { or } \quad \Delta(Z)=Z \otimes Z,
\end{array}
$$

where $Z=\left(z_{j}^{i}\right)_{d \times d}$.

Proposition 3.12. Let $\mathcal{A}=A(V, R)$ be an $N$-homogeneous $\mathbb{k}$-superalgebra.

(a) With $\Delta$ as comultiplication and $\varepsilon$ as counit, the superalgebra end $\mathcal{A}$ becomes a superbialgebra. Moreover, $\delta_{\mathcal{A}}$ makes $\mathcal{A}$ into a graded right $\underline{\mathrm{end}} \mathcal{A}$-comodule superalgebra.

(b) Let $\mathcal{B}$ be any $\mathbb{k}$-superalgebra and let $\delta: \mathcal{A} \rightarrow \mathcal{A} \otimes \mathcal{B}$ be a morphism of superalgebras satisfying $\delta(V) \subseteq V \otimes \mathcal{B}$. Then there is a unique morphism of superalgebras $\varphi:$ end $\mathcal{A} \rightarrow \mathcal{B}$ such that the following diagram commutes:

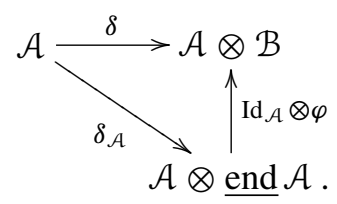

The proposition is proved as in [37], §5, or [7], Theorem 3.

Example 3.13. When $\mathcal{A}=A(V, 0)=\mathrm{T}(V)$, we have end $\mathcal{A}=A\left(V^{*} \otimes V, 0\right)=$ $\mathrm{T}\left(V^{*} \otimes V\right)$; so

$$
\text { end } \mathrm{T}(V)=\mathrm{T}\left(V^{*} \otimes V\right) \text {, }
$$

the free superalgebra generated by the elements $z_{j}^{i}$ in (3.33). 
Example 3.14. By Examples 3.3 and 3.8, we have

$$
\text { end } S_{N}(V)=A\left(V^{*} \otimes V, c_{\pi_{N}}\left(\left(1-c_{Y_{N}}\right)\left(V^{* \otimes N}\right) \otimes c_{Y_{N}}\left(V^{\otimes N}\right)\right)\right)
$$

For example, the algebra end $S_{2}(V)$ is generated by the elements $z_{j}^{i}$ with parity $\hat{i}+\hat{j}$ subject to the relations

$$
\left[z_{j_{1}}^{i_{1}}, z_{j_{2}}^{i_{2}}\right]+(-1)^{\hat{i_{1}} \hat{i_{2}}+\left(\hat{i_{1}}+\hat{i_{2}}\right) \hat{j_{1}}}\left[z_{j_{1}}^{i_{2}}, z_{j_{2}}^{i_{1}}\right]=0
$$

where $[\cdot, \cdot]$ is the supercommutator (2.2). This algebra is highly noncommutative, even for a pure even space $V$.

Let $\mathcal{O}(\mathrm{E}(V))=\mathrm{S}\left(V^{*} \otimes V\right)$ be the supercommutative superbialgebra as in $§ 2.5 .3$, with generators $x_{j}^{i}$. There is a map of superbialgebras

$$
\varphi: \underline{\text { end }} \mathrm{S}_{N}(V) \rightarrow \mathcal{O}(\mathrm{E}(V)), \quad z_{j}^{i} \mapsto x_{j}^{i} .
$$

Indeed, write $\mathcal{B}=\mathcal{O}(\mathrm{E}(V))$ for brevity and recall the coaction $\delta: V \rightarrow V \otimes \mathcal{B}$, $x_{j} \mapsto \sum_{i} x_{i} \otimes x_{j}^{i}$, from (2.11). Since $c_{Y_{N}} \in \operatorname{End}_{\text {comod }_{\mathcal{B}}^{s}}\left(V^{\otimes N}\right)$ (see 2.6 .2$)$, the map $\delta$ extends to a map of superalgebras

$$
\delta: \mathrm{S}_{N}(V) \rightarrow \mathrm{S}_{N}(V) \otimes \mathcal{B} .
$$

Therefore, Proposition 3.12 (b) yields the desired $\varphi$. Note that the coaction of end $\mathrm{S}_{N}(V)$ on $V$, when restricted along $\varphi$, becomes the canonical coaction of $\mathcal{O}(\mathrm{E}(V))$ on $V$; see (2.11) and (3.34).

\section{4. $N$-Koszul superalgebras}

Throughout this section, we fix an $N$-homogeneous superalgebra $\mathcal{A}=A(V, R)$.

4.1. The graded dual $\mathcal{A}^{! *}$. The graded dual

$$
\mathcal{A}^{! *}=\bigoplus_{n} \mathcal{A}_{n}^{! *}
$$

of $\mathcal{A}^{!}$has a natural structure of a graded right end $\mathcal{A}$-comodule. Indeed, the linear dual $\mathcal{A}_{n}^{! *}$ of the degree $n$-component of $\mathcal{A}^{!}$embeds into $V^{\otimes n}$ as follows. Recall from (3.11) that

$$
\mathcal{A}_{n}^{! *}= \begin{cases}V^{\otimes n} & \text { if } n<N, \\ \bigcap_{i+j+N=n} V^{\otimes i} \otimes R \otimes V^{\otimes j} & \text { if } n \geq N .\end{cases}
$$

This identification makes the graded dual $\mathcal{A}^{! *}$ into a graded right end $\mathcal{A}$-comodule. For, by (3.32) the coaction $\delta_{\mathcal{A}}$ restricts in degree 1 to a map $V \rightarrow \overline{V \otimes}$ end $\mathcal{A}$ which 
makes $\mathrm{T}(V)$ into a graded right end $\mathcal{A}$-comodule superalgebra. The structure map $\mathrm{T}(V) \rightarrow \mathrm{T}(V) \otimes \underline{\text { end }} \mathcal{A}$ sends $R \rightarrow R \otimes \underline{\text { end }} \mathcal{A}$. Therefore, each $V^{\otimes i} \otimes R \otimes V^{\otimes j}$ is a end $\mathcal{A}$-subcomodule of $V^{\otimes(i+j+N)}$, and hence $\mathcal{A}_{n}^{! *}$ is a end $\mathcal{A}$-subcomodule of $V^{\otimes n}$. Finally, for all $n \geq 0$,

$$
\mathcal{A}_{n+1}^{! *} \subseteq V \otimes \mathcal{A}_{n}^{! *} \quad \text { and } \quad \mathcal{A}_{n+N}^{! *} \subseteq V^{\otimes N} \otimes \mathcal{A}_{n}^{! *} \cap R \otimes V^{\otimes n}=R \otimes \mathcal{A}_{n}^{! *} .
$$

4.2. The Koszul complex. The map

$$
\begin{aligned}
\mathcal{A} \otimes V^{\otimes(i+1)} & \rightarrow \mathcal{A} \otimes V^{\otimes i}, \\
a \otimes\left(v_{1} \otimes \cdots \otimes v_{i+1}\right) & \mapsto a v_{1} \otimes\left(v_{2} \otimes \cdots \otimes v_{i+1}\right),
\end{aligned}
$$

is a morphism in the category $\operatorname{Comod}_{\text {end } \mathcal{A}}^{s}$ of right end $\mathcal{A}$-comodules, because the end $\mathcal{A}$-coaction $\delta_{\mathcal{A}}$ in (3.31) is a superalgebra map. Furthermore, this map is a left $\mathcal{A}$-module map which preserves total degree, and it restricts to a map of end $\mathcal{A}$-subcomodules

$$
d: \mathcal{A} \otimes \mathcal{A}_{i+1}^{! *} \rightarrow \mathcal{A} V \otimes \mathcal{A}_{i}^{! *} \hookrightarrow \mathcal{A} \otimes \mathcal{A}_{i}^{! *}
$$

which is the $\mathcal{A}$-linear extension of the embedding (4.2). The map $d^{N}$ sends $\mathcal{A}_{i+N}^{! *}$ to $\mathcal{A} R \otimes \mathcal{A}_{i}^{! *}=0 ;$ so $d^{N}=0$. In other words, we have an $N$-complex

$$
K(\mathcal{A}): \cdots \stackrel{d}{\rightarrow} \mathcal{A} \otimes \mathcal{A}_{i+1}^{! *} \stackrel{d}{\rightarrow} \mathcal{A} \otimes \mathcal{A}_{i}^{! *} \stackrel{d}{\rightarrow} \cdots \stackrel{d}{\rightarrow} \mathcal{A} \rightarrow 0
$$

in Comod end $\mathcal{A}^{S}$ consisting of graded-free left $\mathcal{A}$-modules and $\mathcal{A}$-module maps which preserve total degree. Therefore, $K(\mathcal{A})$ splits into a direct sum of $N$-complexes $K(\mathcal{A})^{n}=\bigoplus_{i+j=n} \mathcal{A}_{i} \otimes \mathcal{A}_{j}^{! *}$ in $\operatorname{comod}_{\text {end } \mathcal{A}}^{S}$.

Following [7], the Koszul complex $\mathrm{K}(\overline{\mathcal{A}})$ defined by Berger in [5] can be described as the following contraction of $K(\mathcal{A})$ :

$$
\mathrm{K}(\mathcal{A}): \cdots \stackrel{d^{N-1}}{\longrightarrow} \mathcal{A} \otimes \mathcal{A}_{N+1}^{! *} \stackrel{d}{\rightarrow} \mathcal{A} \otimes \mathcal{A}_{N}^{! *} \stackrel{d^{N-1}}{\longrightarrow} \mathcal{A} \otimes \mathcal{A}_{1}^{! *} \stackrel{d}{\rightarrow} \mathcal{A} \rightarrow 0 .
$$

This is an ordinary complex in Comod end $\mathcal{A}^{S}$ which splits into a direct sum of complexes $\mathrm{K}(\mathcal{A})^{n}$ in $\operatorname{comod}_{\text {end }}^{s} \mathcal{A}$. The $i^{\text {th }}$ components of $\mathrm{K}(\mathcal{A})$ and of $\mathrm{K}(\mathcal{A})^{n}$ are given by

$$
\mathrm{K}(\mathcal{A})_{i}=\mathcal{A} \otimes \mathcal{A}_{v(i)}^{! *} \quad \text { and } \quad \mathrm{K}(\mathcal{A})_{i}^{n}=\mathcal{A}_{n-v(i)} \otimes \mathcal{A}_{v(i)}^{! *}
$$

with $v(i)=v_{N}(i)$ as in equation $(\star)$ in the Introduction. The differential on $\mathrm{K}(\mathcal{A})$ is

$$
\delta_{i}: \mathrm{K}(\mathcal{A})_{i} \rightarrow \mathrm{K}(\mathcal{A})_{i-1} \quad \text { where } \delta_{i}= \begin{cases}d^{N-1} & \text { for } i \text { even, } \\ d & \text { for } i \text { odd }\end{cases}
$$

Writing $\mathcal{A}_{+}=\bigoplus_{n>0} \mathcal{A}_{n}=\mathcal{A} V$ as usual, we have

$\operatorname{Ker} \delta_{i} \subseteq \mathcal{A}_{+} \mathrm{K}(\mathcal{A})_{i}$ 
for all $i$. Indeed, this is clear for odd $i$, since $\delta_{i}=d$ is injective on $\mathcal{A}_{v(i)}^{!^{*}}$. For even $i$, the restriction of $\delta_{i}=d^{N-1}$ to $\mathcal{A}_{v(i)}^{! *}$ is given by $d^{N-1}: \mathcal{A}_{v(i)}^{!^{*}}=\mathcal{A}_{v(i-1)+N-1}^{!^{*}} \hookrightarrow$ $V^{\otimes(N-1)} \otimes \mathcal{A}_{v(i-1)}^{! *} \stackrel{\sim}{\longrightarrow} \mathcal{A}_{N-1} \otimes \mathcal{A}_{v(i-1)}^{! *} \hookrightarrow \mathcal{A} \otimes \mathcal{A}_{v(i-1)}^{! *}$ where the first embedding comes from (4.2).

Since $\mathcal{A}_{v(1)}^{! *}=\mathcal{A}_{1}^{! *}=V$ and $\mathcal{A}_{v(2)}^{! *}=\mathcal{A}_{N}^{! *}=R$ by (4.1), the start of the Koszul complex, augmented by the canonical map

$$
\mathcal{A} \rightarrow \mathbb{k}=\mathcal{A} / \mathcal{A}_{+}
$$

is as follows:

$$
\mathcal{A} \otimes R \stackrel{\delta_{2}}{\rightarrow} \mathcal{A} \otimes V \stackrel{\delta_{1}=\text { mult }}{\longrightarrow} \mathcal{A} \rightarrow \mathbb{k} \rightarrow 0
$$

This piece is easily seen to be exact: writing $\mathcal{A}=\mathrm{T}(V) / I$ with $I=(R)=I \otimes V+$ $\mathrm{T}(V) \otimes R$ as in equation $(\star \star)$ in the Introduction, the map $\mathrm{T}(V)_{+}=\mathrm{T}(V) \otimes V \rightarrow$ $\mathcal{A} \otimes V \stackrel{\delta_{1}}{\rightarrow} \mathcal{A}_{+}$has kernel $I$. Thus, $\operatorname{Ker} \delta_{1}=I / I \otimes V=\operatorname{Im} \delta_{2}$. Hence (4.5) is the start of the minimal graded-free resolution of the left $\mathcal{A}$-module $\mathbb{k}$.

4.3. $N$-homogeneous Koszul superalgebras. Recall from the Introduction that an $N$-homogeneous superalgebra $\mathcal{A}$ is called $N$-Koszul if $\operatorname{Tor}_{i}^{\mathcal{A}}(\mathbb{k}, \mathbb{k})$ is concentrated in degree $v_{N}(i)$ for all $i \geq 0$. By [5], Proposition 2.12, or [8], Theorem 2.4, this happens exactly if the $\operatorname{Koszul}$ complex $\mathrm{K}(\mathcal{A})$ is exact in degrees $i>0$ and in view of (4.5), this amounts to exactness of $\mathrm{K}(\mathcal{A})$ in degrees $i \geq 2$. In this case,

$$
\mathrm{K}(\mathcal{A}) \rightarrow \mathbb{k} \rightarrow 0
$$

is the minimal graded-free resolution of the trivial left $\mathcal{A}$-module $\mathrm{k}$.

The Yoneda Ext-algebra $E(\mathcal{A})=\bigoplus_{i \geq 0} \operatorname{Ext}_{\mathcal{A}}^{i}(\mathbb{k}, \mathbb{k})$ of an $N$-Koszul superalgebra $\mathcal{A}$ has the following description in terms of the dual algebra $\mathcal{A}^{!}$:

$$
\operatorname{Ext}_{\mathcal{A}}^{i}(\mathrm{k}, \mathrm{k}) \cong \mathcal{A}_{v(i)}^{!} \quad(i \geq 0) \text {. }
$$

Moreover, identifying $\operatorname{Ext}_{\mathcal{A}}^{i}(\mathbb{k}, \mathbb{k})$ and $\mathcal{A}_{v(i)}^{!}$, the Yoneda product $f \cdot g$ and the $\mathcal{A}^{!}$product $f g$ for $f \in \operatorname{Ext}_{\mathcal{A}}^{i}(\mathbb{k}, \mathbb{k})=\mathcal{A}_{v(i)}^{!}$and $g \in \operatorname{Ext}_{\mathcal{A}}^{j}(\mathbb{k}, \mathbb{k})=\mathcal{A}_{v(j)}^{!}$are related by $f \cdot g=(-1)^{i j} f g$ when $N=2$, and

$$
f \cdot g= \begin{cases}f g & \text { if } i \text { or } j \text { is even, } \\ 0 & \text { if } i \text { and } j \text { are both odd }\end{cases}
$$

for $N>2$; see [21], Theorem 9.1, [8], Proposition 3.1.

Example 4.1. Quadratic algebras having a PBW-basis are 2-Koszul; see, e.g., [41], Chap. 4, Theorem 3.1. This applies in particular to quantum superspace $\mathcal{A}=A_{\boldsymbol{q}}^{\boldsymbol{f}}$; 
see Example 3.1. A PBW-basis in this case is given by the collection of ordered monomials $x_{1}^{m_{1}} x_{2}^{m_{2}} \ldots x_{d}^{m_{d}}$ with $m_{i} \geq 0$ for all $i$ and $m_{i} \leq 1$ if $\hat{i}=\overline{1}$, as in Example 3.1. For a more general result, see [41], Chap. 4, Theorem 8.1.

Example 4.2. The unmixed Yang-Mills algebras $\mathcal{A}=\operatorname{y\mathcal {M}}^{ \pm}$(see Example 3.2) were shown to be 3-Koszul in [10], [11]. Indeed, letting $\mathcal{A}[\ell]$ denotes the shift of $\mathcal{A}$ that is defined by $\mathcal{A}[\ell]_{n}=\mathcal{A}_{\ell+n}$, the defining relations for $\mathcal{A}$ in the form (3.7) imply that the following complex of graded-free left $\mathcal{A}$-modules is exact:

$$
0 \rightarrow \mathcal{A}[-4] \stackrel{\cdot Y}{\longrightarrow} \mathcal{A}[-3]^{d} \stackrel{\cdot M}{\longrightarrow} \mathcal{A}[-1]^{d} \stackrel{\cdot Y^{\mathrm{tr}}}{\longrightarrow} \mathcal{A} \rightarrow \mathbb{k} \rightarrow 0 .
$$

The piece $\mathcal{A}[-3]^{d} \stackrel{\cdot M}{\longrightarrow} \mathcal{A}[-1]^{d} \stackrel{\cdot Y^{\mathrm{tr}}}{\longrightarrow} \mathcal{A} \rightarrow \mathbb{k} \rightarrow 0$ is identical with (4.5). Therefore, (4.6) is the minimal graded-free resolution of $\mathbb{k}$. The resolution shows that each $\operatorname{Tor}_{i}^{\mathcal{A}}(\mathbb{k}, \mathbb{k})$ is concentrated in degree $v_{3}(i)$, and hence $\mathcal{A}$ is 3-Koszul. It also follows that (4.6) is isomorphic to $\mathrm{K}(\mathcal{A}) \rightarrow \mathbb{k} \rightarrow 0$. In particular, (4.6) confirms the dimensions of the corresponding components $\mathcal{A}_{n}^{!}$in Example 3.7. As has been pointed out in [10], [11], it follows from (4.6) that the Hilbert series $H_{\mathcal{A}}(t)=\sum_{n \geq 0} \operatorname{dim}_{\mathbb{k}} \mathcal{A}_{n} t^{n}$ of $\mathcal{A}=\mathcal{y \mathcal { N }}^{ \pm}$has the form

$$
H_{\mathcal{A}}(t)=\frac{1}{1-d t+d t^{3}-t^{4}}=\frac{1}{\left(1-t^{2}\right)\left(1-d t+t^{2}\right)} .
$$

If $d>2$ then the series has a pole in the interval $(0,1)$, and hence $\operatorname{dim}_{\mathbb{k}} \mathcal{A}_{n}$ grows exponentially with $n$. Therefore, $\mathcal{A}$ is not noetherian in this case; see Stephenson and Zhang [42].

The mixed Yang-Mills algebras $\mathcal{A}=\operatorname{y\mathcal {M}}^{p \mid q}$ with $p \neq 0$ and $q \neq 0$, on the other hand, are never 3-Koszul. For $\mathcal{Y \mathcal { M }}^{1 \mid 1}$ this follows from the description given in Example 3.2: this algebra has infinite global dimension. In general, one can check that the so-called extra condition (see (4.10) below) fails for $\mathcal{A}$, and so $\mathcal{A}$ cannot be Koszul by [5], Prop. 2.7.

Example 4.3. It has been shown in [5], Theorem 3.13, that the $N$-symmetric algebra $\mathrm{S}_{N}(V)$ of a pure even space $V$ over a field of characteristic 0 is $N$-Koszul. An extension of this result will be offered in Theorem 4.5 below.

4.4. Confluence and Koszulity. For the convenience of the reader, we recall the notions of reduction operators and confluence and their relation to the Koszul property. Complete details can be found in Berger [4], [5].

Let $V$ be in Vect $t_{\mathbb{k}}^{s}$ with a graded basis $X=\left\{x_{1}, \ldots x_{d}\right\}$ that is ordered by $x_{1}>x_{2}>\cdots>x_{d}$. The tensors ("monomials") $x_{i}=x_{i_{1}} \otimes x_{i_{2}} \otimes \cdots \otimes x_{i_{N}}$ for $\boldsymbol{i}=\left(i_{1}, i_{2}, \ldots, i_{N}\right) \in\{1,2, \ldots, d\}^{N}$ form a basis of $V^{\otimes N}$ which will be given the lexicographical ordering. An $X$-reduction operator on $V^{\otimes N}$ is a projection 
$S \in \operatorname{End}_{V_{\text {ect }}^{S}}\left(V^{\otimes N}\right)$ such that either $S\left(x_{i}\right)=x_{i}$ or $S\left(x_{i}\right)<x_{i}$ holds for each $\boldsymbol{i}$, where the latter inequality means that $S\left(x_{i}\right)$ is a linear combination (possibly 0 ) of monomials $<x_{i}$. The monomials $x_{i}$ satisfying $S\left(x_{i}\right)=x_{i}$ are called $S$-reduced, all other monomials are $S$-nonreduced. We denote by $\operatorname{Red}(S)$ and $\operatorname{NRed}(S)$ ) the (super) subspaces of $V^{\otimes N}$ that are generated by the $S$-reduced monomials and the $S$-nonreduced monomials, respectively; so $V^{\otimes N}=\operatorname{Red}(S) \oplus \operatorname{NRed}(S)$ and $\operatorname{Im}(S)=\operatorname{Red}(S)$.

Let $\mathcal{L}_{X}\left(V^{\otimes N}\right)$ denote the collection of all $X$-reduction operators on $V^{\otimes N}$. The proof of [4], Theorem 2.3, shows that the application $S \mapsto \operatorname{Ker}(S)$ is a bijection between $\mathcal{L}_{X}\left(V^{\otimes N}\right)$ and the set of all supersubspaces of $V^{\otimes N}$. Hence $\mathcal{L}_{X}\left(V^{\otimes N}\right)$ inherits a lattice structure: for $S, S^{\prime} \in \mathcal{L}_{X}\left(V^{\otimes N}\right)$ one has $X$-reduction operators $S \wedge S^{\prime}$ and $S \vee S^{\prime}$ on $V^{\otimes N}$ which are defined by

$$
\begin{aligned}
& \operatorname{Ker}\left(S \wedge S^{\prime}\right)=\operatorname{Ker}(S)+\operatorname{Ker}\left(S^{\prime}\right), \\
& \operatorname{Ker}\left(S \vee S^{\prime}\right)=\operatorname{Ker}(S) \cap \operatorname{Ker}\left(S^{\prime}\right) .
\end{aligned}
$$

A pair $\left(S, S^{\prime}\right)$ of $X$-reduction operators is said to be confluent if

$$
\operatorname{Red}\left(S \vee S^{\prime}\right)=\operatorname{Red}(S)+\operatorname{Red}\left(S^{\prime}\right) .
$$

Since the inclusion $\supseteq$ is always true, confluence of $\left(S, S^{\prime}\right)$ is equivalent to the inequality

$$
\operatorname{dim}_{\mathbb{k}} \operatorname{Im}\left(S \vee S^{\prime}\right) \leq \operatorname{dim}_{\mathbb{k}}\left(\operatorname{Im}(S)+\operatorname{Im}\left(S^{\prime}\right)\right) .
$$

Let $n \geq N$. Any $X$-reduction operator $S$ on $V^{\otimes N}$ gives rise to $X$-reduction operators $\bar{S}_{n, i}$ on $V^{\otimes n}$ which are defined by

$$
S_{n, i}:=\operatorname{Id}_{V \otimes i} \otimes S \otimes \operatorname{Id}_{V \otimes j} \quad(i+j+N=n) .
$$

A monomial $x_{i}=x_{i_{1}} \otimes x_{i_{2}} \otimes \cdots \otimes x_{i_{n}}$ of length $n \geq N$ is said to be $S$-reduced if $x_{i}$ is $S_{n, i}$-reduced for all $i$, that is, if every connected submonomial of $x_{i}$ of length $N$ is $S$-reduced.

Now let $\mathcal{A}=A(V, R)$ be an $N$-homogeneous superalgebra, and let $S$ be the $X$-reduction operator on $V^{\otimes N}$ such that $\operatorname{Ker}(S)=R$. The algebra $\mathcal{A}$ is said to be $X$-confluent if the pairs $\left(S_{N+i, i}, S_{N+i, 0}\right)$ of $X$-reduction operators on $V^{\otimes N+i}$ are confluent for $i=1, \ldots, N-1$. By (4.7) this amounts to the inequalities

$$
\operatorname{dim}_{\mathbb{k}} \operatorname{Im}\left(S_{N+i, i} \vee S_{N+i, 0}\right) \leq \operatorname{dim}_{\mathbb{k}}\left(\operatorname{Im}\left(S_{N+i, i}\right)+\operatorname{Im}\left(S_{N+i, 0}\right)\right)
$$

being satisfied for $i=1, \ldots, N-1$.

Following Berger [5], we denote by $\mathcal{T}_{n}$ the lattice of supersubspaces of $V^{\otimes n}$ that is generated by the subspaces

$$
R_{n, i}:=V^{\otimes i} \otimes R \otimes V^{\otimes j}=\operatorname{Ker}\left(S_{n, i}\right) \quad(i+j+N=n) .
$$


The superalgebra $\mathcal{A}$ is said to be distributive if the lattices $\mathcal{T}_{n}$ are distributive for all $n$, that is, $C \cap(D+E)=(C \cap D)+(C \cap E)$ holds for all $C, D, E \in \mathcal{T}_{n}$.

The following proposition states the operative facts concerning Koszulity for our purposes. Part (a) is identical with [5], Thm. 3.11, while (b) is [5], Prop. 3.4.

Proposition 4.4. Let $\mathcal{A}=A(V, R)$ be an $N$-homogeneous superalgebra.

(a) If $\mathcal{A}$ is $X$-confluent for some totally ordered graded basis $X$ of $V$ then $\mathcal{A}$ is distributive. Moreover, let $S$ be the $X$-reduction operator on $V^{\otimes N}$ such that $\operatorname{Ker}(S)=R$. Then, for all $n \geq N$, the classes in $\mathcal{A}$ of the $S$-reduced monomials $x_{i_{1}} \otimes x_{i_{2}} \otimes \cdots \otimes x_{i_{n}}$ with $x_{i_{j}} \in X$ form a $\mathbb{k}$-basis of $\mathcal{A}_{n}$.

(b) Assume that $\mathcal{A}$ is distributive and the following "extra condition" is satisfied:

$$
R_{n+N, 0} \cap R_{n+N, n} \subseteq R_{n+N, n-1} \quad(2 \leq n \leq N-1) .
$$

Then $\mathcal{A}$ is $N$-Koszul.

After these preparations, we are now ready to prove the following result. The quadratic case $N=2$ is due to Gurevich [22]; see also Wambst [44].

Theorem 4.5. Let $N \geq 2$ and $0 \neq q \in \mathbb{k}$ and assume that $[n]_{q} \neq 0$ for all $n \geq 1$. Then, for every Hecke operator $\mathcal{R}$ associated with $q$, the $N$-homogeneous superalgebra $\wedge_{\mathcal{R}, N}$ defined in (3.8) is $N$-Koszul.

Proof. Put $\mathcal{A}=\wedge_{\mathcal{R}, N}$ and recall that $\mathcal{A}=A(V, R)$ with

$$
R=\operatorname{Im} \rho_{N, \mathcal{R}}\left(X_{N}\right) \subseteq V^{\otimes N} .
$$

The extra condition (4.10) is a consequence of equation (1.19). Indeed, (1.19) implies that the spaces $R_{n, i}$ in (4.9) have the form

$$
R_{n, i}=\bigcap_{s=i+1}^{i+N-1} \operatorname{Im}\left(\rho_{n, \mathcal{R}}\left(T_{s}\right)+1\right) \subseteq V^{\otimes n} .
$$

Applying (4.11) with $\rho=\rho_{n+N, \mathcal{R}}$ we see that the left-hand side of (4.10) is identical to

$$
\bigcap_{i=1}^{N-1} \operatorname{Im}\left(\rho\left(T_{i}\right)+1\right) \cap \bigcap_{i=n+1}^{n+N-1} \operatorname{Im}\left(\rho\left(T_{i}\right)+1\right)=\bigcap_{i=1}^{n+N-1} \operatorname{Im}\left(\rho\left(T_{i}\right)+1\right)
$$

where the equality holds because $n+1 \leq N$. The last expression is clearly contained in $\bigcap_{i=n}^{n+N-2} \operatorname{Im}\left(\rho\left(T_{i}\right)+1\right)$, which is identical to the right-hand side of (4.10). This establishes the extra condition (4.10).

In order to prove the distributivity of $\mathcal{A}$, we follow the approach taken in [25]. We first prove the claim for the standard solution $\mathcal{R}^{D J}$, i.e., the operator given in 
Example 1.2 with $d=p$ and $q=0$. As above, fix a basis $X=\left\{x_{1}, \ldots, x_{d}\right\}$ of $V$, ordered by $x_{1}>x_{2}>\cdots>x_{d}$, and consider the basis of $V^{\otimes n}$ consisting of the monomials $x_{\boldsymbol{i}}=x_{i_{1}} \otimes x_{i_{2}} \otimes \cdots \otimes x_{i_{n}}$ for $\boldsymbol{i}=\left(i_{1}, i_{2}, \ldots, i_{n}\right) \in\{1,2, \ldots, d\}^{n}$ with the lexicographical ordering. By equation (1.21), the action of the generators $T_{j}$ of the Hecke algebra $\mathscr{H}=\mathscr{H}_{n, q^{2}}$ on this basis is given by

$$
T_{j}\left(x_{\boldsymbol{i}}\right)= \begin{cases}q^{2} x_{\boldsymbol{i}} & \text { if } i_{j}=i_{j+1}, \\ \left(q^{2}-1\right) x_{\boldsymbol{i}}+q x_{\sigma_{j}(i)} & \text { if } i_{j}<i_{j+1}, \\ q x_{\sigma_{j}(i)} & \text { if } i_{j}>i_{j+1} .\end{cases}
$$

Here, $\sigma_{j}=(j, j+1) \in \mathbb{S}_{n}$ and $\sigma(\boldsymbol{i})=\left(i_{\sigma^{-1}(1)}, i_{\sigma^{-1}(2)}, \ldots, i_{\sigma^{-1}(n)}\right)$ for $\sigma \in \mathbb{S}_{n}$, as in Example 3.3.

We claim that the $\mathscr{H}$-submodule of $V^{\otimes n}$ that is generated by $x_{i}$ is given by

$$
\mathscr{H}\left(x_{i}\right)=\bigoplus_{i^{\prime} \in \subseteq_{n}(i)} \mathbb{k} x_{i^{\prime}}
$$

where $\widetilde{S}_{n}(\boldsymbol{i})$ is the $\widetilde{S}_{n}$-orbit of $\boldsymbol{i}$. Indeed, (4.12) implies that each $T_{\sigma}\left(x_{\boldsymbol{i}}\right)$ with $\sigma \in \widetilde{S}_{n}$ is a linear combination of basis vectors $x_{\boldsymbol{i}^{\prime}}$, with $\boldsymbol{i}^{\prime} \in \widetilde{S}_{n}(\boldsymbol{i})$. Hence, $\subseteq$ certainly holds in (4.13). For the reverse inclusion, let $\boldsymbol{i}^{*}$ denote the unique nondecreasing sequence in $\mathfrak{S}_{n}(\boldsymbol{i})$; so $x_{\boldsymbol{i}^{*}}=\max \left\{x_{\boldsymbol{i}^{\prime}} \mid \boldsymbol{i}^{\prime} \in \mathfrak{S}_{n}(\boldsymbol{i})\right\}$. The last formula in (4.12) implies that

$$
T\left(x_{i}\right)=q^{r(i)} x_{i}
$$

where $T$ is a suitable finite product of length $r(i) \geq 0$ in the generators $T_{j}$. Since $T$ is a unit in $\mathscr{H}$, the inclusion $\supseteq$ holds in (4.13), thereby proving the asserted equality.

Furthermore, (4.14) and (1.18) (with $q$ replaced by $q^{2}$ ) give

$$
q^{r(i)} X_{n}\left(x_{i}\right)=X_{n}\left(x_{i}^{*}\right) .
$$

These elements are nonzero. For, (4.15) implies that the elements $X_{n}\left(x_{i} *\right)$ span the image of $X_{n}$ on $V^{\otimes n}$, and their number is $\left(\begin{array}{c}d+n-1 \\ n\end{array}\right)$ which is equal to the rank of $X_{n}$ (cf. [25], Eq. (5)). It follows that $X_{n}\left(V^{\otimes n}\right)^{n}=\operatorname{Im} \rho_{n, \mathcal{R} D J}\left(X_{n}\right)$ has a k-basis consisting of the elements

$$
\left\{X_{n}\left(x_{\boldsymbol{i}^{*}}\right) \mid \boldsymbol{i}^{*}=\left(i_{1} \leq i_{2} \leq \cdots \leq i_{n}\right) \in\{1,2, \ldots, d\}^{n}\right\} .
$$

Next, writing

$$
X_{n}\left(x_{i}\right)=\sum_{i^{\prime} \in \mathbb{S}_{n}(i)} \lambda_{\boldsymbol{i}^{\prime}} x_{\boldsymbol{i}^{\prime}}
$$

with $\lambda_{\boldsymbol{i}^{\prime}} \in \mathbb{k}$, we claim that

$$
\lambda_{\sigma_{j}\left(\boldsymbol{i}^{\prime}\right)}= \begin{cases}\lambda_{\boldsymbol{i}^{\prime}} & \text { if } \boldsymbol{i}^{\prime}=\sigma_{j}\left(\boldsymbol{i}^{\prime}\right) \\ q^{ \pm 1} \lambda_{\boldsymbol{i}^{\prime}} & \text { otherwise }\end{cases}
$$


To prove this, we may assume that $\boldsymbol{i}^{\prime} \neq \sigma_{j}\left(\boldsymbol{i}^{\prime}\right)$. We compute the coefficient of $x_{\sigma_{j}\left(\boldsymbol{i}^{\prime}\right)}$ in $T_{j} X_{n}\left(x_{i}\right)$ in two ways: by (1.18) this coefficient is equal to $q^{2} \lambda_{\sigma_{j}\left(i^{\prime}\right)}$ while (4.12) yields the expression $q \lambda_{\boldsymbol{i}^{\prime}}+\left(q^{2}-q^{1 \pm 1}\right) \lambda_{\sigma_{j}\left(\boldsymbol{i}^{\prime}\right)}$. The claim follows from this. Writing an arbitrary $\sigma \in \mathbb{S}_{n}$ as a product of the inversions $\sigma_{j}$, we see that the coefficients $\lambda_{\boldsymbol{i}^{\prime}}$ in (4.16) only differ by a nonzero scalar, and hence they are all nonzero since $X_{n}\left(x_{i}\right) \neq 0$.

By Proposition 4.4, it suffices to check the $X$-confluence conditions (4.8) for $i=1, \ldots, N-1$. So let $S$ be the $X$-reduction operator on $V^{\otimes N}$ with $\operatorname{Ker}(S)=$ $R$. It is easy to see from the discussion above (with $n=N$ ) that $S$ is given by $S\left(x_{\boldsymbol{i}^{*}}\right)=\left(1-X_{N} / \lambda_{\boldsymbol{i}^{*}}\right)\left(x_{\boldsymbol{i}} *\right)$ and $S\left(x_{\boldsymbol{i}}\right)=x_{\boldsymbol{i}}$ for $\boldsymbol{i} \neq \boldsymbol{i}^{*}$. According to (4.11) and the discussion above, the dimension of $\left(R \otimes V^{\otimes i}\right) \cap\left(V^{\otimes i} \otimes R\right)$ is $\left(\begin{array}{c}d+N+i-1 \\ N+i\end{array}\right)$. Thus, the dimension of the left-hand side of (4.8) is $d^{N+i}-\left(\begin{array}{c}d+N+i-1 \\ N+i\end{array}\right)$. On the other hand the monomials in $V^{\otimes N+i}$ that belong to $\operatorname{NRed}\left(S_{N+i, i}\right) \cap \operatorname{NRed}\left(S_{N+i, 0}\right)$ are exactly those of the form $x_{\boldsymbol{i}} *$ with $\boldsymbol{i}^{*} \in\{1, \ldots, d\}^{N+i}$ non-decreasing. Their number is precisely $\left(\begin{array}{c}d+N+i-1 \\ N+i\end{array}\right)$. Therefore, the dimension of $\operatorname{Im}\left(S_{N+i, i}\right)+\operatorname{Im}\left(S_{N+i, 0}\right)=$ $\operatorname{Red}\left(S_{N+i, i}\right)+\operatorname{Red}\left(S_{N+i, 0}\right)$ is at least $d^{N+i}-\left(\begin{array}{c}d+N+i-1 \\ N+i\end{array}\right)$. This proves the inequality in (4.8), thereby finishing the proof of the theorem for the case $\mathcal{R}=\mathcal{R}^{D J}$.

In order to deal with an arbitrary Hecke operator $\mathcal{R}$, recall that $\mathscr{H}_{n, q}$ is split semisimple, having a representative set of simple modules $M_{\lambda}$ indexed by the partitions $\lambda \vdash n$; see (1.15). We denote the representation of $\mathscr{H}_{n, q}$ on $M_{\lambda}$ by $\rho_{\lambda}$; it does not depend on the operator $\mathcal{R}$ but only on the partition $\lambda$.

Let us fix a decomposition

$$
V^{\otimes n}=\bigoplus_{t \in T} M_{t}
$$

into simple $\mathscr{H}_{n, q}$-submodules $M_{t}$. Since all $M_{t}$ are invariant under the operators $\rho_{n, \mathcal{R}}\left(T_{j}\right)$, formula (4.11) yields the decomposition

$$
R_{n, i}=\bigoplus_{t \in T} \bigcap_{s=i+1}^{i+N-1}\left(\rho_{n, \mathcal{R}}\left(T_{s}\right)+1\right)\left(M_{t}\right)=\bigoplus_{t \in T} R_{n, i} \cap M_{t}
$$

for all $i$. Thus, by [25], Lemma 1.2, distributivity of the lattice $\mathcal{T}_{n}$ that is generated by the subspaces $R_{n, i}$ of $V^{\otimes n}$ is equivalent to distributivity of the lattices $\mathcal{T}_{n} \cap M_{t}$ $(t \in T)$ that are generated by the subspaces

$$
R_{n, i} \cap M_{t}=\bigcap_{s=i+1}^{i+N-1}\left(\rho_{n, \mathcal{R}}\left(T_{s}\right)+1\right)\left(M_{t}\right)
$$

of $M_{t}$. Now, each $M_{t}$ is isomorphic to $M_{\lambda}$ for some $\lambda \vdash n$. Therefore, the lattice $\tau_{n} \cap M_{t}$ is isomorphic to the lattice of subspaces of $M_{\lambda}$ that is generated by the 
subspaces

$$
\bigcap_{s=i+1}^{i+N-1}\left(\rho_{\lambda}\left(T_{S}\right)+1\right)\left(M_{\lambda}\right)
$$

with $i+N \leq n$. Finally, when $d=\operatorname{dim} V>n$, then all simple $\mathscr{H}_{n, q}$-modules $M_{\lambda}$ appear in $V^{\otimes n}$; see [15], Proposition 5.1. Thus, the distributivity of the lattice associated to $\mathcal{R}^{D J}$, which we have already verified, implies the distributivity of the corresponding lattice for any Hecke operator $\mathcal{R}$. This completes the proof.

\section{Koszul duality and master theorem}

In this section, $\mathcal{A}=A(V, R)$ denotes an $N$-homogeneous superalgebra that is assumed to be $N$-Koszul $(N \geq 2)$.

5.1. By Koszulity, the complexes

$$
\mathrm{K}(\mathcal{A})^{n}: \cdots \rightarrow \mathcal{A}_{n-v_{N}(i)} \otimes \mathcal{A}_{v_{N}(i)}^{! *} \rightarrow \mathcal{A}_{n-v_{N}(i-1)} \otimes \mathcal{A}_{v_{N}(i-1)}^{!^{*}} \rightarrow \ldots \rightarrow \mathcal{A}_{n} \rightarrow 0
$$

are exact for $n>0$. This yields equations in the Grothendieck ring $R_{\text {end }} \mathcal{A}$ of the category comod $\underline{\underline{\text { end }} \mathcal{A}}$ :

$$
\sum_{i \geq 0}(-1)^{i}\left[\mathcal{A}_{n-v_{N}(i)}\right]\left[\mathcal{A}_{v_{N}(i)}^{! *}\right]=0 \quad(n>0) .
$$

In the power series ring $R_{\underline{\text { end }}} \mathcal{A} \llbracket t \rrbracket$ over the Grothendieck ring $\mathrm{R}_{\underline{\text { end }}} \mathcal{A}$, define the Poincaré series

$$
P_{\mathcal{A}}(t)=\sum_{n \geq 0}\left[\mathcal{A}_{n}\right] t^{n} \quad \text { and } \quad P_{\mathcal{A} ! *}(t)=\sum_{n \geq 0}\left[A_{n}^{!^{*}}\right] t^{n} .
$$

For any power series $P(t)=\sum_{n} a_{n} t^{n}$, we use the notation

$$
P_{N}(t):=\sum_{n \equiv 0,1 \bmod N}(-1)^{\alpha_{N}(n)} a_{n} t^{n}
$$

where $\alpha_{N}(n)=n-(n \bmod N)$ denotes the largest multiple of $N$ less than or equal to $n$. Thus, $P_{2}(t)=P(t)$ and in general

$$
P_{N}(-t)=\sum_{n \equiv 0,1 \bmod N}(-1)^{n \bmod N} a_{n} t^{n}=\sum_{i \geq 0}(-1)^{i} a_{v_{N}(i)} t^{v_{N}(i)}
$$

In particular,

$$
P_{\mathcal{A}^{! *, N}}(-t)=\sum_{i \geq 0}(-1)^{i}\left[\mathcal{A}_{v_{N}(i)}^{! *}\right] t^{v_{N}(i)} .
$$

Equations (5.1) are equivalent to the following Koszul duality formula: 
Proposition 5.1. For any $N$-homogeneous Koszul superalgebra $\mathcal{A}$, the identity

$$
P_{\mathcal{A}}(t) P_{\mathcal{A}^{! *}, N}(-t)=1
$$

holds in $R_{\text {end }} \mathcal{A} \llbracket t \rrbracket$.

Applying the ring homomorphism $\left.\chi^{s} \llbracket t \rrbracket: R_{\text {end } \mathcal{A} \llbracket t \rrbracket \rightarrow(\text { end } \mathcal{A})}\right)_{\overline{0}} \llbracket t \rrbracket$, where $\chi^{s}$ is the supercharacter map as in Corollary 2.3, the formula in Proposition 5.1 takes the following form in $(\underline{\text { end }} \mathcal{A}) \overline{0} \llbracket t \rrbracket$ :

\section{Corollary 5.2.}

$$
\left(\sum_{\ell} \chi_{\mathcal{A}_{\ell}}^{s} t^{\ell}\right) \cdot\left(\sum_{m \equiv 0,1 \bmod N}(-1)^{m \bmod N} \chi_{\mathcal{A}_{m}^{s *}}^{s} t^{m}\right)=1 .
$$

Analogous formulas hold with the supercharacter $\chi^{s}$ replaced by the ordinary character $\chi$ or by one of the dimensions dim and sdim.

By (3.32) the coaction of end $\mathcal{A}$ on $\mathcal{A}$ sends $\mathcal{A}_{n}$ to $\mathcal{A}_{n} \otimes(\text { end } \mathcal{A})_{n}$. A similar remark holds for the end $\mathcal{A}$-coaction on $\mathcal{A}^{! *}$; see $\S 4.1$. Therefore, both factors in Corollary 5.2 actually belong to the Rees subring $\prod_{n \geq 0} B_{n} t^{n}$ of $B \llbracket t \rrbracket$, where we have put $B=(\underline{\text { end }} \mathcal{A})_{\overline{0}}$.

Example 5.3. As an application of the Hilbert series version of Corollary 5.2, we see that the duals $\mathcal{A}^{!}$of the Yang-Mills algebras $\mathcal{A}=\operatorname{y\mathcal {N}}^{p \mid q}$ are never 3-Koszul. In fact, by Example 3.7, we have $H_{\mathcal{A}^{!}}(t)=1+d t+d^{2} t^{2}+d t^{3}+t^{4}$ if $p=0$ or $q=0$ and $H_{\mathcal{A}^{!}}(t)=1+d t+d^{2} t^{2}+d t^{3}$ otherwise. In either case, $H_{\mathcal{A}^{!}}(t)^{-1}$ has a nonzero coefficient at $t^{5}$, which rules out Koszulity.

5.2. A master theorem modeled on the $N$-symmetric superalgebra $\mathbf{S}_{N}(V)$. We put $\mathcal{A}=\mathrm{S}_{N}(V)$ and use the notation of Examples 3.3 and 3.8. In particular, we assume that char $\mathrm{k}=0$ and work with a fixed basis $x_{1}, \ldots, x_{d}$ of $V=\mathcal{A}_{1}$ so that $\hat{i}=\overline{0}$ for $i \leq p$ and $\hat{i}=\overline{1}$ for $i>p$.

From Example 3.3 (see also Proposition 4.4 (a)), we know that a basis of $\mathcal{A}_{\ell}$ is given by the monomials $x_{i}=x_{i_{1}} x_{i_{2}} \ldots x_{i_{\ell}}$ for sequences $i=\left(i_{1}, \ldots, i_{\ell}\right) \in\{1, \ldots, d\}^{\ell}$ such that $\boldsymbol{i}$ has no connected subsequence $\boldsymbol{j}=\left(j_{1}, \ldots, j_{N}\right)$ of length $N$ satisfying

$$
1 \leq j_{1}<\cdots<j_{m} \leq p<j_{m+1} \leq \cdots \leq j_{N} \leq d=p+q
$$

for some $m$. Adapting notation of Etingof and Pak [16] to our setting, we denote this set of sequences $\boldsymbol{i}$ by

$$
\Lambda(p \mid q, N)_{\ell} .
$$

For example, $\Lambda(p \mid q, 2)_{\ell}$ consists of all weakly decreasing sequences $\boldsymbol{i}=\left(i_{1}, \ldots, i_{\ell}\right)$ with entries from $\{1, \ldots, d\}$ and such that no repetition occurs in the range $\{p+1, \ldots, d\}$. 
In order to evaluate the character $\chi_{\mathcal{A}_{\ell}}^{s}$ in Corollary 5.2, recall from (3.34) that the coaction $\delta_{\mathcal{A}}: \mathcal{A} \rightarrow \mathcal{A} \otimes \underline{\text { end }} \mathcal{A}$ is given on the generators $x_{i}$ of $\mathcal{A}$ by

$$
\delta_{\mathcal{A}}\left(x_{i}\right)=\sum_{j} x_{j} \otimes z_{i}^{j} \in \mathcal{A} \otimes \underline{\text { end }} \mathcal{A}
$$

where $z_{i}^{j}=x^{j} \otimes x_{i}$ are the canonical generators of the algebra end $\mathcal{A}$. For $\boldsymbol{i}=$ $\left(i_{1}, \ldots, i_{\ell}\right) \in \Lambda(p \mid q, N)_{\ell}$, we have

$$
\delta_{\mathcal{A}}\left(x_{\boldsymbol{i}}\right)=\delta_{\mathcal{A}}\left(x_{i_{1}}\right) \delta_{\mathcal{A}}\left(x_{i_{2}}\right) \ldots \delta_{\mathcal{A}}\left(x_{i_{\ell}}\right) \in \mathcal{A}_{\ell} \otimes \underline{\text { end }} \mathcal{A} .
$$

Since $\mathcal{A}_{\ell} \otimes \underline{\text { end }} \mathcal{A}=\bigoplus_{i \in \Lambda(p \mid q, N)_{\ell}} x_{i} \otimes \underline{\text { end }} \mathcal{A}$, we can define $Z(i) \in(\underline{\text { end }} \mathcal{A})_{\overline{0}}$ by

$$
\delta_{\mathcal{A}}\left(x_{\boldsymbol{i}}\right)=x_{\boldsymbol{i}} \otimes Z(\boldsymbol{i})+\left(\text { terms supported on } \Lambda(p \mid q, N)_{\ell} \backslash\{\boldsymbol{i}\}\right) .
$$

Then (2.8) becomes

$$
\chi_{\mathcal{A}_{\ell}}^{s}=\sum_{i \in \Lambda(p \mid q, N)_{\ell}}(-1)^{\hat{i}} Z(\boldsymbol{i})
$$

with $\hat{i}=\hat{i_{1}}+\cdots+\hat{i_{\ell}}$.

Now consider the superbialgebra $\mathcal{B}=\mathcal{O}(\mathrm{E}(V))=\mathbb{k}\left[x_{j}^{i} \mid 1 \leq i, j \leq d\right]$ defined in $\S 2.5 .3$ and recall that the $x_{j}^{i}$ are supercommuting variables of parity $\hat{i}+\hat{j}$ over $\mathbb{k}$. Restricting the comodule $\mathcal{A}_{\ell}$ to $\mathcal{B}$ along the map $\varphi$ : end $S_{N}(V) \rightarrow \mathcal{B}, z_{j}^{i} \mapsto x_{j}^{i}$ in (3.35) we must replace $Z(\boldsymbol{i})$ in (5.4) by $X(\boldsymbol{i}):=\varphi\left(\overline{Z(\boldsymbol{i}))} \in \mathcal{B}_{\overline{0}}\right.$. Thus, writing

$$
y_{i}=\sum_{j} x_{j} \otimes x_{i}^{j} \in \mathcal{A} \otimes \mathcal{B}
$$

and $y_{\boldsymbol{i}}=y_{i_{1}} \ldots y_{i_{\ell}} \in \mathcal{A}_{\ell} \otimes \mathcal{B}=\bigoplus_{\boldsymbol{j} \in \Lambda(p \mid q, N)_{\ell}} x_{\boldsymbol{j}} \otimes \mathcal{B}$ for $\boldsymbol{i}=\left(i_{1}, \ldots, i_{\ell}\right)$, we have

$$
y_{\boldsymbol{i}}=x_{\boldsymbol{i}} \otimes X(\boldsymbol{i})+\left(\text { terms supported on } \Lambda(p \mid q, N)_{\ell} \backslash\{\boldsymbol{i}\}\right) .
$$

As for the supercharacter of $\mathcal{A}_{m}^{! *}$, recall from (4.1) and (3.15) that, for all $n \geq N$,

$$
\mathcal{A}_{n}^{! *}=\bigcap_{i+j+N=n} V^{\otimes i} \otimes R \otimes V^{\otimes j}=\Lambda^{n} V .
$$

Viewing $\mathcal{A}_{n}^{! *}=\Lambda^{n} V$ as a comodule over $\mathcal{B}=\mathcal{O}(\mathrm{E}(V))$, the supercharacter of $\mathcal{A}_{n}^{! *}$ is the $n^{\text {th }}$ elementary supersymmetric function $e_{n}$ which we know, by Proposition 2.4, to be identical to the coefficient at $t^{n}$ of the characteristic function ber $(1+t X)$ of the generic supermatrix $X=\left(x_{j}^{i}\right)_{1 \leq i, j \leq d}$ of type $p \mid q$; so the diagonal blocks $X_{11}=\left(x_{j}^{i}\right)_{1 \leq i, j \leq p}$ and $X_{22}=\left(x_{j}^{i}\right)_{p+1 \leq i, j \leq p+q}$ consist of even entries while all other entries are odd.

To summarize, we obtain the following super-version of [16], Theorem 2. 
Theorem 5.4. Let $X=\left(x_{j}^{i}\right)_{d \times d}$ be the generic supermatrix of type $p \mid q$. Then

$$
\left(\sum_{\ell} \sum_{i \in \Lambda(p \mid q, N)_{\ell}}(-1)^{\hat{i}} X(i) t^{\ell}\right) \cdot\left(\sum_{m \equiv 0,1 \bmod N}(-1)^{m \bmod N} e_{m} t^{m}\right)=1
$$

holds in the power series ring $\mathbb{k}\left[x_{j}^{i} \mid \text { all } i, j\right]_{\overline{0}} \llbracket t \rrbracket$. Here $\Lambda(p \mid q, N)_{\ell}$ and $X(i)$ are defined by (5.3) and (5.5), respectively, and the $e_{m}$ are the coefficients of the characteristic function ber $(1+t X)=\sum_{n \geq 0} e_{n} t^{n}$ of $X$.

5.3. As an application of Theorem 5.4, we determine the superdimension Hilbert series

$$
H_{\mathcal{A}}^{s}(t)=\sum_{\ell \geq 0} \operatorname{sdim}_{\mathbb{k}} \mathcal{A}_{\ell} t^{\ell}
$$

for the $N$-symmetric superalgebra $\mathcal{A}=\mathrm{S}_{N}(V)$. For the pure even case, this was already done by Etingof and Pak [16]. The notations of $\$ 5.2$ remain in effect.

In view of Corollary 2.3, the superdimension Poincaré series follows by applying the counit $\varepsilon: \mathcal{B} \rightarrow \mathbb{k}$ to the equation in Theorem 5.4. Indeed, by (2.11), the counit $\varepsilon$ sends $X \mapsto 1_{d \times d}$, and hence the elements $X(\boldsymbol{i})$ in (5.5) all map to 1 . Therefore, the first factor in Theorem 5.4 becomes

$$
H_{\mathcal{A}}^{s}(t)=\sum_{\ell \geq 0}\left(\sum_{i \in \Lambda(p \mid q, N)_{\ell}}(-1)^{\hat{i}}\right) t^{\ell}
$$

For the second factor, note that

$$
\operatorname{ber}\left(1+t 1_{d \times d}\right)=(1+t)^{p-q}
$$

by (2.12). Thus,

$$
\begin{aligned}
H_{\mathcal{A}}^{s}(t) & =\sum_{\ell \geq 0}\left(\sum_{i \in \Lambda(p \mid q, N)_{\ell}}(-1)^{\hat{\boldsymbol{i}}}\right) t^{\ell} \\
& = \begin{cases}\left(\sum_{m \equiv 0,1 \bmod N}(-1)^{m \bmod N}\left(\begin{array}{c}
p-q \\
m
\end{array}\right) t^{m}\right)^{-1} & \text { if } p \geq q, \\
\left(\sum_{m \equiv 0,1 \bmod N}(-1)^{\alpha_{N}(m)}\left(\begin{array}{c}
m+q-p-1 \\
q-p-1
\end{array}\right) t^{m}\right)^{-1} & \text { if } p<q,\end{cases}
\end{aligned}
$$

where $\alpha_{N}(m)=m-(m \bmod N)$ denotes the largest multiple of $N$ less than or equal to $m$ as in $\$ 5.1$.

5.4. The ordinary Hilbert series $H_{\mathcal{A}}(t)=\sum_{\ell \geq 0} \operatorname{dim}_{\mathbb{k}} \mathcal{A}_{\ell} t^{\ell}$ of the $N$-symmetric superalgebra $\mathcal{A}=\mathrm{S}_{N}(V)$ is as follows. Recall from $\S 5.2$ that

$$
\operatorname{dim}_{\mathbb{k}} \mathcal{A}_{\ell}=\left|\Lambda(p \mid q, N)_{\ell}\right|
$$


and from (3.16) that

$$
\operatorname{dim}_{\mathbb{k}} \mathcal{A}_{n}^{!}= \begin{cases}d^{n} & \text { if } n<N, \\
\sum_{r+s=n}\left(\begin{array}{l}
p \\
r
\end{array}\right)\left(\begin{array}{c}
q+s-1 \\
s
\end{array}\right) & \text { if } n \geq N .\end{cases}
$$

Therefore, the Hilbert series is

$$
\begin{aligned}
H_{\mathcal{A}}(t) & =\sum_{\ell \geq 0}\left|\Lambda(p \mid q, N)_{\ell}\right| t^{\ell} \\
& =\left(\sum_{m=0,1 \bmod N}(-1)^{m \bmod N}\left(\sum_{r+s=m}\left(\begin{array}{c}
p \\
r
\end{array}\right)\left(\begin{array}{c}
q+s-1 \\
s
\end{array}\right)\right) t^{m}\right)^{-1} .
\end{aligned}
$$

5.5. Less is known about the Hilbert series of the $N$-homogeneous superalgebras $\mathcal{A}=\Lambda_{\mathcal{R}, N}$ associated to an arbitrary Hecke operator $\mathcal{R}: V^{\otimes 2} \rightarrow V^{\otimes 2}$ on a vector superspace $V$; see Example 3.4. Recall that $\mathcal{A}=A(V, R)$ with $R=\operatorname{Im} \rho_{\mathcal{R}}\left(X_{N}\right) \subseteq$ $V^{\otimes N}$. For any $N$-homogeneous algebra $\mathcal{A}=A(V, R)$, we have

$$
\operatorname{dim}_{\mathbb{k}} \mathcal{A}_{n}^{!}=\operatorname{dim}_{\mathbb{k}} \bigcap_{i+j+N=n} V^{\otimes j} \otimes R \otimes V^{\otimes i}
$$

by (3.10) and (3.11). For $R=\operatorname{Im} \rho_{\mathcal{R}}\left(X_{N}\right)$ in particular, (1.19) further implies that

$$
\bigcap_{i+j+N=n} V^{\otimes j} \otimes R \otimes V^{\otimes i}=\rho_{\mathcal{R}}\left(X_{n}\right)\left(V^{\otimes n}\right)
$$

holds for $n \geq N$. Now [26], Theorem 3.5, implies that

$$
H_{\wedge_{\mathcal{R}, 2} !}(t)=\frac{\prod_{\ell=1}^{r}\left(1+a_{\ell} t\right)}{\prod_{m=1}^{s}\left(1-b_{m} t\right)},
$$

where $(r, s)$ is the birank of $\mathcal{R}$ and $a_{\ell}$ and $b_{m}$ are positive real numbers. For example, in the situation of 5.4, $(r, s)=(p, q)$ and $a_{\ell}=b_{m}=1$.

For any complex power series $P(t)$, the power series $P_{N}(-t)$ in (5.2) can be written as

$$
P_{N}(-t)=\frac{1}{N} \sum_{i=1}^{N-1}\left(1-\zeta_{N}^{-i}\right) P\left(\zeta_{N}^{i} t\right)
$$

where $\zeta_{N}=e^{2 \pi i / N}$. In particular,

$$
\begin{aligned}
H_{\mathcal{A}^{! *}, N}(-t) & =\frac{1}{N} \sum_{i=1}^{N-1}\left(1-\zeta_{N}^{-i}\right) \frac{\prod_{\ell=1}^{r}\left(1+a_{\ell} \zeta_{N}^{i} t\right)}{\prod_{m=1}^{s}\left(1-b_{m} \zeta_{N}^{i} t\right)} \\
& =\frac{Q_{N, \boldsymbol{a}, \boldsymbol{b}}(t)}{\prod_{m=1}^{s}\left(1+b_{m} t+\cdots+b_{m}^{N-1} t^{N-1}\right)}
\end{aligned}
$$


for some real polynomial $Q_{N, \boldsymbol{a}, \boldsymbol{b}}(t)$ with coefficients being polynomial in $\boldsymbol{a}=\left(a_{\ell}\right)$ and $\boldsymbol{b}=\left(b_{m}\right)$. Therefore, the Hilbert series of $\mathcal{A}$ has the form

$$
H_{\mathcal{A}}(t)=\frac{\prod_{m=1}^{s}\left(1+b_{m} t+\cdots+b_{m}^{N-1} t^{N-1}\right)}{Q_{N, \boldsymbol{a}, \boldsymbol{b}}(t)} .
$$

Notice that the fraction on the right-hand side is reduced.

In particular, (5.7) has the form

$$
H_{\mathcal{A}}(t)=\frac{\left(1-t^{N}\right)^{s}}{(1-t)^{s} Q_{N, \mathbf{1}, \mathbf{1}}(t)} .
$$

\section{Appendix}

For lack of a suitable reference, we include here a proof of the proposition that was stated in the Introduction. Our proof is based on the proof of [8], Proposition 2.1, and on additional details that were communicated to us by Roland Berger. For the basics concerning graded algebras, we refer the reader to [9], Chap. II §11, or [6].

As in the Introduction, $\mathcal{A}=\bigoplus_{n \geq 0} \mathcal{A}_{n}$ denotes an arbitrary connected $\mathbb{Z}_{\geq 0}$-graded kk-algebra and $V$ is a graded subspace of $\mathcal{A}_{+}=\bigoplus_{n>0} \mathcal{A}_{n}$ satisfying $\mathcal{A}_{+}=V \oplus \mathcal{A}_{+}^{2}$. Thus, $\mathrm{T}(V) / I \stackrel{\sim}{\longrightarrow} \mathcal{A}$ for some graded ideal $I$ of $\mathrm{T}(V)$. For convenience, we state the proposition again:

Proposition. The relation ideal I of $\mathcal{A}$ lives in degrees $\geq N$ if and only if $\operatorname{Tor}_{i}^{\mathcal{A}}(\mathbb{k}, \mathbb{k})$ lives in degrees $\geq v_{N}(i)= \begin{cases}\frac{i}{2} N & \text { if } i \text { is even, } \\ \frac{i-1}{2} N+1 & \text { if } i \text { is odd. }\end{cases}$

Proof. Let

$$
P: \cdots \rightarrow P_{i} \stackrel{d_{i}}{\longrightarrow} P_{i-1} \stackrel{d_{i-1}}{\longrightarrow} \ldots \stackrel{d_{1}}{\longrightarrow} P_{0} \stackrel{d_{0}}{\longrightarrow} \mathbb{k} \rightarrow 0
$$

be a minimal graded-free resolution of the trivial left $\mathcal{A}$-module k. Thus, all $P_{i}$ have the form $P_{i}=\mathcal{A} \otimes E_{i}$ for some graded subspace $E_{i} \subseteq \operatorname{Ker} d_{i-1}$ which is chosen so that

$$
\operatorname{Ker} d_{i-1}=E_{i} \oplus \mathcal{A}_{+} \operatorname{Ker} d_{i-1} .
$$

In particular, we may take $E_{0}=\mathbb{k}$ and $E_{1}=V$. The differential $d_{i}: P_{i} \rightarrow P_{i-1}$ is the graded $\mathcal{A}$-module map that is defined by the inclusion $E_{i} \hookrightarrow P_{i-1}$. By the graded Nakayama Lemma (e.g., [9], Prop. 6, p. AII.171) our choice of $E_{i}$ implies that

$$
\operatorname{Im} d_{i}=\mathcal{A} E_{i}=\operatorname{Ker} d_{i-1} \quad \text { and } \quad \operatorname{Ker} d_{i} \subseteq \mathcal{A}_{+} \otimes E_{i}=\mathcal{A}_{+} P_{i}
$$


for all $i$. Consequently, the complex $\mathbb{k} \otimes_{\mathcal{A}} P$ has zero differential, and hence

$$
\operatorname{Tor}_{i}^{\mathcal{A}}(\mathbb{k}, \mathbb{k}) \cong \mathbb{k} \otimes_{\mathcal{A}} P_{i} \cong E_{i} .
$$

In particular,

$$
\operatorname{Tor}_{0}^{\mathcal{A}}(\mathbb{k}, \mathbb{k}) \cong \mathbb{k} \text { and } \operatorname{Tor}_{1}^{\mathcal{A}}(\mathbb{k}, \mathbb{k}) \cong V=\mathcal{A}_{+} / \mathcal{A}_{+}^{2}
$$

live in degrees $0=v_{N}(0)$ and $\geq 1=v_{N}(1)$, respectively. Moreover, the kernel of $d_{1}: P_{1}=(\mathrm{T}(V) / I) \otimes V \rightarrow P_{0}=\mathcal{A}$ is exactly $I / I \otimes V$, and so

$$
\operatorname{Tor}_{2}^{\mathcal{A}}(\mathbb{k}, \mathbb{k}) \cong \operatorname{Ker} d_{1} / \mathcal{A}_{+} \operatorname{Ker} d_{1} \cong I /(V \otimes I+I \otimes V) .
$$

Thus, $I$ lives in degrees $\geq N$ if and only if $\operatorname{Tor}_{2}^{\mathcal{A}}(\mathbb{k}, \mathbb{k})$ lives in degrees $\geq N=v_{N}(2)$.

For the remainder of the proof, assume that $I$ lives in degrees $\geq N$. We will show by induction on $i$ that $\operatorname{Tor}_{i}^{\mathcal{A}}(\mathbb{k}, \mathbb{k})=E_{i}$ lives in degrees $\geq v_{N}(i)$ for all $i$. The cases $i \leq 2$ have been checked above. Assume that $E_{i}$ lives in degrees $\geq v_{N}(i)$ and similarly for $E_{i-1}$. By (A.2), we know that $E_{i+1} \subseteq \operatorname{Ker} d_{i} \subseteq \mathcal{A}_{+} \otimes E_{i}$ and so $E_{i+1}$ certainly lives in degrees $\geq v_{N}(i)+1$. Since $v_{N}(i)+1=v_{N}(i+1)$ when $i$ is even (or when $i$ is arbitrary and $N=2$ ), we are done in these cases. From now on, we assume that $i$ is odd. We must show that $E_{i+1}$ lives in degrees $\geq v_{N}(i+1)=\frac{i+1}{2} N$. Since $E_{i+1} \subseteq \operatorname{Ker} d_{i}$, it suffices to show that $d_{i}$ is injective in degrees $<\frac{i+1}{2} N$, and since $E_{i}$ lives in degrees $\geq v_{N}(i)=\frac{i-1}{2} N+1$, our goal is to show that $d_{i}$ is injective on all homogeneous components $P_{i, n}$ of $P_{i}$ in degrees $n=\frac{i-1}{2} N+j$ with $j=1, \ldots, N-1$. Put $m=\frac{i-1}{2} N$ for simplicity and note that

$$
P_{i, m+j}=\bigoplus_{\ell=1}^{j} \mathcal{A}_{j-\ell} \otimes E_{i, m+\ell}
$$

and

$$
P_{i-1, m+j}=\bigoplus_{k=0}^{j} \mathcal{A}_{j-k} \otimes E_{i-1, m+k}
$$

since $E_{i-1}$ lives in degrees $\geq v_{N}(i-1)=m$. The proposition will be a consequence of the following claims:

(a) $d_{i}$ is injective on all summands $\mathcal{A}_{j-\ell} \otimes E_{i, m+\ell}$ in (A.3), and

(b) the subspaces $d_{i}\left(\mathcal{A}_{j-\ell} \otimes E_{i, m+\ell}\right)=\mathcal{A}_{j-\ell} E_{i, m+\ell}$ for $\ell=1, \ldots, j$ form a direct sum inside $P_{i-1, m+j}$.

In order to prove (a), recall that the restriction of $d_{i}$ to $E_{i, m+\ell}$ is the inclusion

$$
E_{i, m+\ell} \hookrightarrow P_{i-1, m+\ell}=\bigoplus_{k=0}^{\ell} \mathcal{A}_{\ell-k} \otimes E_{i-1, m+k} .
$$


Hence, the effect of $d_{i}$ on the $\ell^{\text {th }}$ summand in (A.3) is the embedding

$$
\mathcal{A}_{j-\ell} \otimes E_{i, m+\ell} \hookrightarrow \bigoplus_{k=0}^{\ell} \mathcal{A}_{j-\ell} \otimes \mathcal{A}_{\ell-k} \otimes E_{i-1, m+k},
$$

followed by the map

$$
\bigoplus_{k=0}^{\ell} \mathcal{A}_{j-\ell} \otimes \mathcal{A}_{\ell-k} \otimes E_{i-1, m+k} \rightarrow \bigoplus_{k=0}^{\ell} \mathcal{A}_{j-k} \otimes E_{i-1, m+k} \subseteq P_{i-1, m+j}
$$

which is given by the multiplication map $\mathcal{A}_{j-\ell} \otimes \mathcal{A}_{\ell-k} \rightarrow \mathcal{A}_{j-k}$. Since $j-k<N$, our hypothesis on $I$ implies that $\mathcal{A}_{j-k} \cong \mathrm{T}(V)_{j-k}$, and similarly $\mathcal{A}_{j-\ell} \cong \mathrm{T}(V)_{j-\ell}$ and $\mathcal{A}_{\ell-k} \cong \mathrm{T}(V)_{\ell-k}$. Therefore, the above multiplication map is identical with the injection $\mathrm{T}(V)_{j-\ell} \otimes \mathrm{T}(V)_{\ell-k} \hookrightarrow \mathrm{T}(V)_{j-k}$ in $\mathrm{T}(V)$. This proves (a).

For (b), we proceed by induction on $j$. The case $j=1$ being obvious, let $1 \leq$ $j \leq N-2$ and assume that (ii) holds for $1, \ldots, j$. We wish to show that the subspaces $\mathcal{A}_{j+1-\ell} E_{i, m+\ell}(\ell=1, \ldots, j+1)$ of $P_{i-1, m+j+1}$ form a direct sum. First, by (A.1) we have $E_{i, m+j+1} \cap \mathcal{A}_{+} \operatorname{Ker} d_{i-1}=0$ while $\sum_{\ell=1}^{j} \mathcal{A}_{j+1-\ell} E_{i, m+\ell} \subseteq \mathcal{A}_{+} \operatorname{Ker} d_{i-1}$. Therefore, it suffices to show that the sum $\sum_{\ell=1}^{j} \mathcal{A}_{j+1-\ell} E_{i, m+\ell}$ is direct. To this end, note that $\mathcal{A}_{j+1-\ell}=\sum_{d \geq 1} V_{d} \mathcal{A}_{j+1-d-\ell}$ holds for all $\ell \leq j$. Hence,

$$
\sum_{\ell=1}^{j} \mathcal{A}_{j+1-\ell} E_{i, m+\ell}=\sum_{d \geq 1} V_{d} \sum_{\ell=1}^{j} \mathcal{A}_{j+1-d-\ell} E_{i, m+\ell} .
$$

By induction, $\sum_{\ell=1}^{j} \mathcal{A}_{j+1-d-\ell} E_{i, m+\ell}$ is a direct sum inside $P_{i-1, m+j+1-d}$. Thus, it suffices to show that the sum $\sum_{d \geq 1} V_{d} P_{i-1, m+j+1-d} \subseteq P_{i-1, m+j+1}$ is direct. But (A.4) gives

$$
P_{i-1, m+j+1}=\bigoplus_{k=0}^{j+1} \mathcal{A}_{j+1-k} \otimes E_{i-1, m+k}=\bigoplus_{k=0}^{j+1} \mathrm{~T}(V)_{j+1-k} \otimes E_{i-1, m+k},
$$

where the last equality holds since all $j+1-k<N$. Therefore,

$$
\sum_{d \geq 1} V_{d} P_{i-1, m+j+1-d}=\bigoplus_{d \geq 1} V_{d} \otimes \bigoplus_{k=0}^{j+1-d} \mathrm{~T}(V)_{j+1-d-k} \otimes E_{i-1, m+k}
$$

as desired. This proves (b), thereby completing the proof of the proposition.

Acknowledgement. The authors wish to thank Roland Berger for his helpful comments throughout the completion of this paper. Work on this article was initiated during a visit of ML to the Université Montpellier 2 in June 2006. ML would like to thank Claude Cibils for arranging this visit and for his warm hospitality in Montpellier, and James Zhang for help with some examples in this article. 


\section{References}

[1] M. Artin and W. F. Schelter, Graded algebras of global dimension 3. Adv. Math. 66 (1987), 171-216. Zbl 0633.16001 MR 917738

[2] A. Beilinson, V. Ginzburg, and W. Soergel, Koszul duality patterns in representation theory. J. Amer. Math. Soc. 9 (1996), 473-527. Zbl 0864.17006 MR 1322847

[3] F. A. Berezin, Introduction to superanalysis. Math. Phys. Appl. Math. 9, D. Reidel Publishing Co., Dordrecht 1987. Zbl 0659.58001 MR 914369

[4] R. Berger, Confluence and Koszulity. J. Algebra 201 (1998), 243-283. Zbl 0915.16025 MR 1608711

[5] R. Berger, Koszulity for nonquadratic algebras. J. Algebra 239 (2001), 705-734. Zbl 1035.16023 MR 1832913

[6] R. Berger, Dimension de Hochschild des algèbres graduées. C. R. Acad. Sci. Paris Sér. I Math. 341 (2005), 597-600. Zbl 1082.16009 MR 2179797

[7] R. Berger, M. Dubois-Violette, and M. Wambst, Homogeneous algebras. J. Algebra 261 (2003), 172-185. Zbl 1061.16034 MR 1967160

[8] R. Berger and N. Marconnet, Koszul and Gorenstein properties for homogeneous algebras. Algebr. Represent. Theory 9 (2006), 67-97. Zbl 05052958 MR 2233117

[9] N. Bourbaki, Algèbre, Chapitres 1 à 3, Hermann, Paris 1970. Zbl 0211.02401 MR 0274237

[10] A. Connes and M. Dubois-Violette, Yang-Mills algebra. Lett. Math. Phys. 61 (2002), 149-158. Zbl 1028.53025 MR 1936574

[11] A. Connes and M. Dubois-Violette, Yang-Mills and some related algebras. In Rigorous quantum field theory, Progr. Math. 251, Birkhäuser, Basel 2007, 65-78. MR 2279211

[12] P. Deligne and J. S. Milne, Tannakian categories. In Hodge cycles, motives, and Shimura varieties. Lecture Notes in Math. 900, Springer-Verlag, Berlin 1982, 101-228. Zbl 0477.14004 MR 0654325

[13] R. Dipper and G. James, Representations of Hecke algebras of general linear groups. Proc. London Math. Soc. (3) 52 (1986), 20-52. Zbl 0587.20007 MR 812444

[14] R. Dipper and G. James, $q$-tensor space and $q$-Weyl modules. Trans. Amer. Math. Soc. 327 (1991), 251-282. Zbl 0798.20009 MR 1012527

[15] J. Du, B. Parshall, and J. P. Wang, Two-parameter quantum linear groups and the hyperbolic invariance of $q$-Schur algebras. J. London Math. Soc. (2) 44 (1991), 420-436. Zbl 0694.22014 MR 1149005

[16] Pavel Etingof and Igor Pak, An algebraic extension of the MacMahon Master Theorem. Proc. Amer. Math. Soc., to appear.

[17] D. Foata and G.-N. Han, A basis for the right quantum algebra and the " $1=q$ " principle. Preprint 2006. arXiv:math.CO/0603463

[18] D. Foata and G.-N. Han, A new proof of the Garoufalidis-Lê-Zeilberger Quantum MacMahon Master Theorem. J. Algebra 307 (2007), 424-431. Zbl 1108.05014 MR 2278064 
[19] D. Foata and G.-N. Han, Specializations and extensions of the quantum MacMahon Master Theorem. Linear Algebra Appl. 423 (2007), 445-455. Zbl 1115.05007 MR 2312419

[20] S. Garoufalidis, T. T. Q. Lê, and D. Zeilberger, The quantum MacMahon Master Theorem. Proc. Natl. Acad. Sci. USA 103 (2006), 13928-13931. MR 2270337

[21] E. L. Green, E. N. Marcos, R. Martínez-Villa, and P. Zhang, D-Koszul algebras. J. Pure Appl. Algebra 193 (2004), 141-162. Zbl 1075.16013 MR 2076383

[22] D. I. Gurevich, Algebraic aspects of the quantum Yang-Baxter equation. Algebra i Analiz 2 (1990), 119-148; English transl. Leningrad Math. J. 2 (1991), 801-828. Zbl 0728.17012 MR 1080202

[23] D. I. Gurevich, P. N. Pyatov, and P. A. Saponov, The Cayley-Hamilton theorem for quantum matrix algebras of GL $(m \mid n)$ type. Algebra i Analiz 17 (2005), 160-182; English transl. St. Petersburg Math. J. 17 (2006), 119-135. Zbl 05044646 MR 2140677

[24] D. I. Gurevich, P. N. Pyatov, and P. A. Saponov, Quantum matrix algebras of GL $(m \mid n)$ type: the structure of the characteristic subalgebra and its spectral parametrization. Teoret. Mat. Fiz. 147 (2006), 14-46; English transl. Theoret. and Math. Phys. 147 (2006), 460-485. MR 2254713

[25] P. H. Hai, Koszul property and Poincaré series of matrix bialgebras of type $A_{n}$. J. Algebra 192 (1997), 734-748. Zbl 0874.17008 MR 1452685

[26] P. H. Hai, Poincaré series of quantum spaces associated to Hecke operators. Acta Math. Vietnam. 24 (1999), 235-246. Zbl 0949.16025 MR 1710780

[27] P. H. Hai, Realizations of quantum hom-spaces, invariant theory, and quantum determinantal ideals. J. Algebra 248 (2002), 50-84. Zbl 1033.17021 MR 1879007

[28] P. H. Hai and M. Lorenz, Koszul algebras and the quantum MacMahon Master Theorem, Bull. London Math. Soc. 39 (2007), 667-676. Zbl 05190255 MR 2346948

[29] C. Kassel, Quantum groups. Graduate Texts in Math. 155, Springer-Verlag, New York 1995. Zbl 0808.17003 MR 1321145

[30] H. M. Khudaverdian and T. T. Voronov, Berezinians, exterior powers and recurrent sequences. Lett. Math. Phys. 74 (2005), 201-228. Zbl 1083.58008 MR 2191955

[31] M. Konvalinka, A generalization of Foata's fundamental transformation and its applications to the right-quantum algebra. Preprint 2007. arXiv:math/0703203v1

[32] M. Konvalinka, Non-commutative Sylvester's determinantal identity. Preprint 2007. arXiv:math/0703213v1

[33] M. Konvalinka and I. Pak, Non-commutative extensions of the MacMahon Master Theorem. Adv. Math. 216 (2007), 29-61. Zbl 05201714 MR 2353248

[34] I. G. Macdonald, Symmetric functions and Hall polynomials. 2nd ed., Oxford Math. Monogr., The Clarendon Press, Oxford University Press, Oxford 1995 Zbl 0824.05059 MR 1354144

[35] P. A. MacMahon, Combinatory analysis. Vol. I, II, Cambridge University Press, Cambridge 1915-16; reprinted in one volume by Chelsea Publishing Co., New York 1960. JFM 45.1271.01 JFM 46.0118.07 Zbl 0101.25102 MR 0141605 
[36] Y. I. Manin, Some remarks on Koszul algebras and quantum groups. Ann. Inst. Fourier (Grenoble) 37 (1987), 191-205. Zbl 0625.58040 MR 927397

[37] Y. I. Manin, Quantum groups and non-commutative geometry, Université de Montréal, Centre de Recherches Mathématiques, Montreal, QC, 1988. Zbl 0724.17006 MR 1016381

[38] Y. I. Manin, Multiparametric quantum deformation of the general linear supergroup. Comm. Math. Phys. 123 (1989), 163-175. Zbl 0673.16004 MR 1002037

[39] Y. I. Manin, Topics in noncommutative geometry, M. B. Porter Lectures, Princeton University Press, Princeton, NJ, 1991. Zbl 0724.17007 MR 1095783

[40] Y. I. Manin, Gauge field theory and complex geometry. 2nd ed., Grundlehren Math. Wiss. 289, Springer-Verlag, Berlin 1997. Zbl 0884.53002 MR 1632008

[41] A. Polishchuk and L. Positselski, Quadratic algebras. Univ. Lecture Ser. 37, Amer. Math. Soc., Providence, RI, 2005. Zbl 02242607 MR 2177131

[42] D. R. Stephenson and J. J. Zhang, Growth of graded Noetherian rings. Proc. Amer. Math. Soc. 125 (1997), 1593-1605. Zbl 0879.16011 MR 1371143

[43] G. M. Tuynman, Supermanifolds and supergroups. Math. Appl. 570, Kluwer Academic Publishers, Dordrecht 2004. Zbl 1083.58001 MR 2102797

[44] M. Wambst, Complexes de Koszul quantiques. Ann. Inst. Fourier (Grenoble) 43 (1993), 1089-1156. Zbl 0810.16010 MR 1252939

Received April 15, 2007

P. H. Hai, Fachbereich Mathematik, Universität Duisburg-Essen, 45117 Essen, Germany, and Institute of Mathematics, 10307 Hanoi, Vietnam

E-mail: hai.phung@uni-duisburg-essen.de

B. Kriegk, LaMUSE, Faculté des Sciences et Techniques, Université de Saint-Etienne,

23 rue du Docteur Paul Michelon, 42023 Saint-Etienne Cedex 2, France

E-mail: benoit.kriegk@univ-st-etienne.fr

M. Lorenz, Department of Mathematics, Temple University, Philadelphia, PA 19122-6094,

U.S.A.

E-mail: lorenz@temple.edu 